\title{
Secular Evolution in Disk Galaxies
}

To appear in RMP

\author{
J A Sellwood \\ Department of Physics \& Astronomy, Rutgers University, 136 Frelinghuysen Road, Piscataway, NJ 08854, \\ USA
}

\begin{abstract}
Disk galaxies evolve over time through processes that may rearrange both the radial mass profile and the metallicity distribution within the disk. This review of such slow changes is largely, though not entirely, restricted to internally driven processes that can be distinguished from evolution driven by galaxy interactions. It both describes our current understanding of disk evolution and identifies areas where more work is needed. Stellar disks are heated through spiral scattering, which increases random motion components in the plane, while molecular clouds redirect some fraction of the random energy into vertical motion. The recently discovered process of radial migration at the corotation resonance of a transient spiral mode does not alter the underlying structure of the disk, since it neither heats the disk nor causes it to spread, but it does have a profound effect on the expected distribution of metallicities among the disk stars. Bars in disks are believed to be major drivers of secular evolution through interactions with the outer disk and with the halo. Once the material that makes up galaxy disks is converted into stars, their overall angular momentum distribution cannot change by much, but that of the gas is generally far more liable to rearrangement, allowing rings and pseudobulges to form. While simulations are powerful tools from which we have learned a great deal, those of disks may suffer from collisional relaxation that requires some results to be interpreted with caution.
\end{abstract}

\section{INTRODUCTION}

Galaxies are distributed throughout the Universe in a clustering hierarchy. A large majority of bright galaxies are disk-shaped, with a significant minority being ellipsoidal. The question of how these objects came into existence is the subject of intense current research. However, it has become increasingly clear that the presentday properties of galaxies were not exclusively laid down at the time of their formation, and that internally-driven processes have contributed significantly to their present properties. This review describes, from a theoretical perspective, the dynamical behavior that is believed to be important in restructuring galaxy disks from their initially endowed properties. Kormendy \& Kennicutt (2004), updated in Kormendy (2012), give a comprehensive review of the same topic from an observer's perspective. The present-day properties of galaxy disks were recently reviewed by van der Kruit \& Freeman (2011).

It has to be said at the outset that galaxies do not first form, and then evolve, in temporally distinct phases. In fact, even today formation is incomplete for many galaxies, such as the Milky Way. However, the balance has clearly shifted from the rapid collapse and merging picture that characterized the roughly first one third of the life of the Universe to more quiescent evolution over the remaining two thirds. The vibrant topic of galaxy formation is too large to be included in any detail in this review, yet it cannot be omitted entirely as it provides the context for galaxy evolution.

After the initial collapse, and every subsequent major merger event, gas begins to settle into a disk whose orien-

*Electronic address: sellwood@physics.rutgers.edu tation is determined by the angular momentum that it acquired from the tidal fields of other nearby mass concentrations. The thinness of galaxy disks requires there to have been a protracted period of quiescent evolution, during which a number of internally-driven processes gradually rearrange the structure of galaxies. These include disk growth through slow accretion of gas, the formation and evolution of bars, recurring spiral instabilities, the response of the stellar system to the radial rearrangement of matter, especially the gas, etc. These, together with the influence of the environment, drive what has become known as "secular evolution," by which is meant the gradual restructuring of a galaxy over time scales much longer than a crossing time. Evolution is mostly driven by the outward redistribution of angular momentum in a galaxy, which enables it to reach a state of lower energy, and such changes are prolonged by gas accretion.

Note that the word "secular" has a narrow meaning in classical studies of rotating fluid spheroids by Maclaurin and Jacobi (summarized by Chandrasekhar, 1987). They revealed that viscosity, a dissipative process, can destabilize some rotating Maclaurin spheroids, which become secularly unstable and evolve to Jacobi ellipsoids. However, the same Maclaurin spheroid could be dynamically stable in the absence of viscosity. In this review, I adopt the deliberately broader and vaguer meaning of secular explained in the previous paragraph.

Of the many processes discussed in this review, I here highlight two of particular significance. Spiral patterns are probably the most important agent of secular evolution. They have long been known to redistribute angular momentum and to cause the random motions of stars to increase over time, but we now know that they cause extensive radial mixing of both the stars and the gas, and they smooth small-scale irregularities in the mass distribution. Bars also cause substantial secular 
changes. Once formed, stellar bars in isolated, gas-free disks simply rotate steadily with no tendency to evolve (e.g. Miller \& Smith, 1979), but interaction with gas and other mass components of the galaxy can gradually alter the properties of the bar, with evolutionary consequences for its host galaxy. It is noteworthy that the rate of secular evolution by both spirals and bars is substantially accelerated by a moderate fraction of gas.

In order to keep to a manageable length, I focus here on secular evolution in galaxy disks largely driven through internal processes. In appropriate places I mention environmental factors, such as the infall of debris, tidal interactions, etc., which can also alter the structure of a galaxy. But describing the full extent to which environmental factors may drive evolution would stray into the domain of galaxy assembly and would add too much to the length of this review.

\section{BACKGROUND}

The purpose of this long section is to introduce the concepts and mechanisms that are invoked in the main part of the review, which begins in §III. Note that I refer back to the various parts of this section where appropriate, so that those who start with the later sections can quickly locate a summary of the background that is assumed.

\section{A. Galaxy formation}

The current paradigm for galaxy formation is the $\Lambda$ CDM (Lambda Cold Dark Matter) model (Springel et al., 2006), which holds that galaxies form in dark matter halos whose distribution and properties were seeded by a Gaussian random field of tiny density fluctuations in the early Universe (Bardeen et al., 1986). Because the mean matter density was so close to the closure density in the early Universe, even very mild initial overdensities grew through self-gravity, and subclumps on all but the very largest scales were gravitationally bound together. In this epoch, few overdense regions were isolated from their neighbors, and the growth of structure was characterized by the development of a "cosmic web" of dense sheets, filaments and voids, that has been simulated with ever increasing precision (see Frenk \& White, 2012, for a recent review). During this phase, initially distinct collapse centers underwent a considerable degree of merging, giving each major overdensity a treelike origin as different leaves, branches and more major boughs join to the main density peak. Later, a little after redshift $z \sim 1$, some mysterious "dark energy", which has many of the properties of Einstein's cosmological constant, has caused the originally slowing universal expansion to reaccelerate (Riess et al., 1998); (Perlmutter et al., 1999). Reacceleration increases the isolation of different pieces of the clustering hierarchy, reducing the later merging rate of halos and allowing galaxies to settle in a more dynamically quiescent period.

As the first collapses began, the mutual tidal stresses between the extended overdense regions impart some angular momentum about each collapse center. A dimensionless measure of the total angular momentum $L$ is $\lambda_{L}=L|E|^{1 / 2} / G M^{5 / 2}$, where $E$ and $M$ are the system's total energy and mass. Halos formed in hierarchical simulations are found to have a log-normal distribution of this parameter with a most probable value of $\lambda_{L, 0}=0.037$ (Bullock et al., 2001). Stochastic hierarchical growth leads to a net angular momentum of a halo that varies in magnitude and direction both with distance from the point of highest density and over time.

The dynamical evolution just described is driven by the dark matter, popularly supposed to be some relic, weakly interacting particle that became nonrelativistic in the early Universe and is therefore described as "cold." It is believed (Hinshaw et al., 2013) to have a cosmic density more than 6 times that of the "baryonic" matter, comprised of the familiar protons, neutrons and electrons. The small mass fraction of hydrogen and helium, which combined from the primordial plasma to become neutral gas at $z \sim 1100$, is known to have subsequently reionized sometime around $z \sim 10$ (Larson et al., 2011) as the first objects dense enough to support thermonuclear reactions began to radiate.

Gas collected in overdense regions, either by cooling of shock heated material or through flows of cold gas accreted along filaments of the cosmic web, and spun up as it settled into rotationally supported disks at the centers of the dark matter halos. The on-going formation of stars in these gaseous disks gave rise to the galaxies we observe today. Numerical simulations of the galaxy formation process lack the dynamic range (Springel et al., 2006) to resolve the complicated gas physics of fragmentation, star formation, and the subsequent release of energy through supernovae (see $\llbracket . \mathrm{I}$ ). Thus the rate, efficiency, and precise location of star formation, described as "subgrid physics," is included in the simulations through rules that are both motivated by observational evidence and tuned to achieve the desired outcome. The difficulty that simulations have in making galaxylike objects with the properties we observe today is widely attributed to inadequacies in the implementation of star formation and feedback.

As halos continue to merge, the disks of stars that had begun to form in them are transformed into amorphous ellipsoidal components in the violently changing gravitational potential (Barnes \& Hernquist, 1992). Where some combination of shocks, supernovae, and active galactic nuclei has heated most remaining gas to very high temperatures, the merged remnants are believed to become the "red sequence" galaxies that have little gas that can cool and reconstitute an active star-forming 
disk. ${ }^{1}$ Where gas can cool and resettle, the ellipsoidal ball of stars becomes a spheroidal bulge at the center of a newly assembling disk that generally continues to grow. Disk galaxies that are actively forming stars are the "blue cloud" galaxies.

This current picture is widely accepted as broadly correct because it accounts for the distribution of galaxies throughout the Universe and some of their properties (Springel et al., 2006). Yet there are quite a number of important predictions of the model that seem to be inconsistent with known galaxy properties. Perhaps the foremost is that (1) many present-day galaxies lack the types of bulges produced by mergers (Shen et al. 2010); (Kormendy et al., 2010), whereas the merging hierarchy of the model predicts substantial, dense bulges in most large galaxies. Other problems are (2) the central density of dark matter in the halos of galaxies today seem less than the models predict (Sellwood, 2009); (Kuzio de Naray \& Spekkens, 2011), (3) the number and properties of dwarf satellite galaxies surrounding each major galaxy seems inconsistent with what we observe (Bovlan-Kolchin et al., 2012), (4) the old stars in galactic disks reside in a thinner layer that cannot have been stirred by a minor merger for a very long period (Wyse, 2009), etc. See Silk \& Mamon (2012) for a more detailed critique.

\section{B. Relaxation time in the disks of galaxies}

Except in the immediate neighborhood of a star, the gravitational attraction of nearby stars is generally negligible compared with that from the large-scale distribution of matter in a galaxy. While the argument that establishes this point can be found in many text books (e.g. Binney \& Tremaine, 2008, hereafter BT08), the usual derivation requires some modification for disk systems that is generally omitted even though it involves several nontrivial issues that are important both to the scattering of stars by mass clumps in the disk and to the proper interpretation of simulations, as noted elsewhere in this review.

\section{Standard theory for spherical systems}

A test particle moving at velocity $\boldsymbol{v}$ along a trajectory that passes a stationary field star of mass $\mu$ with impact parameter $b$ is deflected by the attraction of the field star. For a distant passage, it acquires a transverse velocity component $\left|\boldsymbol{v}_{\perp}\right| \simeq 2 G \mu /(b v)$ to first order

\footnotetext{
1 The terms "red sequence" and "blue cloud" refer to distinct groupings in the color-magnitude diagram for galaxies (e.g. Baldrv et al., 2004) and reflect, more objectively, the distinction between early- and late-type galaxies already known to Hubble (e.g. Sandage, 1961).
}

(BT08 eq. 1.30). Encounters at impact parameters small enough to produce deflections where this approximation fails badly are negligibly rare and relaxation is driven by the cumulative effect of many small deflections.

If the density of field stars is $n$ per unit volume and uniform in $3 \mathrm{D}$, the test particle encounters $\delta n=2 \pi b \delta b n v$ stars per unit time with impact parameters between $b$ and $b+\delta b$. Assuming stars to have equal masses, each encounter at this impact parameter produces a randomly directed $\boldsymbol{v}_{\perp}$ that will cause a mean square net deflection per unit time of

$$
\left\langle\delta v_{\perp}^{2}\right\rangle \simeq\left(\frac{2 G \mu}{b v}\right)^{2} \times 2 \pi b \delta b n v=\frac{8 \pi G^{2} \mu^{2} n}{v} \frac{\delta b}{b} .
$$

The total rate of deflection from all encounters is the integral over impact parameters, yielding

$$
\left\langle v_{\perp}^{2}\right\rangle=\frac{8 \pi G^{2} \mu^{2} n}{v} \int_{b_{\min }}^{b_{\max }} \frac{d b}{b}=\frac{8 \pi G^{2} \mu^{2} n}{v} \ln \Lambda,
$$

where $\ln \Lambda \equiv \ln \left(b_{\max } / b_{\min }\right)$ is the Coulomb logarithm. Typically one chooses the lower limit to be the impact parameter of a close encounter, $b_{\min } \simeq 2 G \mu / v^{2}$, for which $\left|\boldsymbol{v}_{\perp}\right|$ is overestimated by the linear formula, while the upper limit is, say, the half mass or effective radius, $R_{e}$, of the stellar distribution beyond which the density decreases rapidly. The vagueness of these definitions is not of great significance to an estimate of the overall rate because we need only the logarithm of their ratio. The Coulomb logarithm implies equal contributions to the integrated deflection rate from every decade in impact parameter simply because the diminishing gravitational influence of more distant stars is exactly balanced by their increasing numbers.

Note that the first order deflections that give rise to this steadily increasing random energy come at the expense of second order reductions in the forward motion of the same particles that we have neglected (Hénon, 1973). Thus the system does indeed conserve energy, as it must.

We define the relaxation time to be the time needed for $\left\langle v_{\perp}^{2}\right\rangle \simeq v^{2}$, where $v$ is the typical velocity of a star. Thus

$$
\tau_{\text {relax }}=\frac{v^{3}}{8 \pi G^{2} \mu^{2} n \ln \Lambda} .
$$

To order of magnitude, a typical velocity $v^{2} \approx G N \mu / R_{e}$, where $N$ is the number of stars each of mean mass $\mu$, yielding $\Lambda \approx N$. Defining the dynamical time to be $\tau_{\text {dyn }}=R_{e} / v$ and setting $n \sim 3 N /\left(4 \pi R_{e}^{3}\right)$, we have

$$
\tau_{\text {relax }} \approx \frac{N}{6 \ln N} \tau_{\text {dyn }},
$$

which shows that the collisionless approximation is well satisfied in galaxies, which have $10^{8} \lesssim N \lesssim 10^{11}$ stars. Including the effect of a smooth dark matter component in this estimate would increase the typical velocity, $v$, thereby further lengthening the relaxation time. 


\section{Applications to disk systems}

This standard argument, however, assumed a pressuresupported quasispherical system in several places. Rybicki (1972) pointed out that the flattened geometry and organized streaming motion within disks affects the relaxation time in a number of important ways.

First, the assumption that the typical encounter velocity is comparable to the orbital speed $v=\left(G N \mu / R_{e}\right)^{1 / 2}$ is clearly wrong; stars move past each other at the typical random speeds in the disk, say $\beta v$ with $\beta \sim 0.1$, causing larger deflections and decreasing the time for $\left\langle v_{\perp}^{2}\right\rangle \simeq \beta^{2} v^{2}$ by a factor $\beta^{3}$.

Second, the distribution of scatterers is not uniform in $3 \mathrm{D}$, as was implicitly assumed in eq. (1). Assuming a razor-thin disk changes the volume element from $2 \pi v b \delta b$ for $3 \mathrm{D}$ to $2 v \delta b$ in $2 \mathrm{D}$, which changes the integrand in Eq. (2) to $b^{-2}$ and replaces the Coulomb logarithm by the factor $\left(b_{\min }^{-1}-b_{\max }^{-1}\right)$. In $2 \mathrm{D}$ therefore, relaxation is dominated by close encounters when the forces are Newtonian, and the system could never be collisionless.

Real galaxy disks are neither razor thin, nor spherical, so the spherical dependence applies at ranges up to the typical disk thickness $z_{0}$, beyond which the density of stars drops too quickly to make a significant further contribution to the relaxation rate. Thus we should use $\Lambda \simeq z_{0} / b_{\min }$ for disks.

Third, the local mass density is also higher, so that $N \sim \pi R_{e}^{2} z_{0} n$. These three considerations shorten the relaxation time (Eq. 4) by the factor

$$
\beta^{3}\left(\frac{z_{0}}{R_{e}}\right) \frac{\ln \left(R_{e} / b_{\min }\right)}{\ln \left(z_{0} / b_{\min }\right)},
$$

or $\sim 10^{-4}$ for $\beta \simeq 0.1$ and reasonable $z_{0} / R_{e}$. Note that star-star encounters in galaxy disks remain unimportant, even with this large reduction in the relaxation time scale. But significant relaxation can occur in simulations of stellar disks, and the issues originally raised by Rybicki are of importance for scattering by clouds, as developed below.

A fourth consideration for disks is that the mass distribution is much less smooth than is the case in the bulk of pressure-supported galaxies. A galaxy disk generally contains massive star clusters and giant molecular clouds whose influence on the relaxation rate turns out to be non-negligible and also determines the shape of the equilibrium velocity ellipsoid (see \$II.B.4).

\section{Stellar orbits in disks}

While the above considerations should be borne in mind, it is nevertheless useful to regard the gravitational potential of a galaxy as a smooth function of position to a first approximation. If this assumption holds, the stellar component of a galaxy behaves as a collisionless fluid (BT08). I extend the discussion to include relaxation processes present in galaxy disks in $\cong$ III.B.
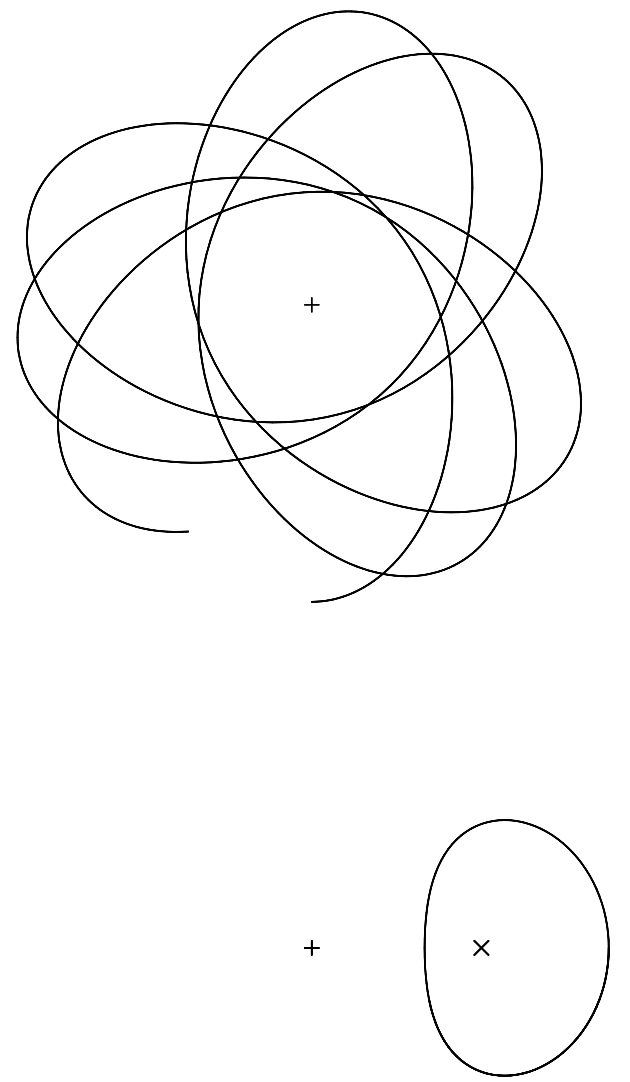

FIG. 1 An eccentric orbit in the midplane of an axisymmetric potential. The center of the potential is marked with a plus. The orbit is drawn in the inertial frame above and below in a frame that rotates with the guiding center, marked with a cross. Since the star conserves $L_{z}$, the motion around the epicycle is in the retrograde sense.

A star, or test particle, moving in the symmetry plane $(z=0)$ of a steady axisymmetric potential $\Phi(R, z)$ must conserve its specific energy $E$ and specific angular momentum $L_{z}$ about the symmetry axis $(R=0)$; these are the only two nontrivial integrals of motion when $L_{x}=L_{y}=0$. In general, the orbit of a star is a rosette, as shown in Fig. 1, but when viewed from a frame rotating about the center of the galaxy at an angular frequency $\Omega_{\phi}$ (lower panel), we see that the motion can be decomposed into a radial oscillation about a guiding center, which is marked with a cross, plus the uniform angular motion of the guiding center about the center with a pe$\operatorname{riod} \tau_{\phi}=2 \pi / \Omega_{\phi}$. The guiding center radius $R_{g}$ is where the radial acceleration of the particle passes through zero, i.e. where the central attraction matches the centripetal acceleration:

$$
\left(\frac{\partial \Phi}{\partial R}\right)_{\left(R_{g}, 0\right)}=\frac{L_{z}^{2}}{R_{g}^{3}} .
$$

For eccentric orbits, such as that shown, $\Omega_{\phi} \lesssim \Omega_{c} \equiv$ $L_{z} / R_{g}^{2}$, the angular frequency of circular motion for the same $L_{z}$. The radial oscillation is anharmonic and we simply define the radial frequency $\Omega_{R} \equiv 2 \pi / \tau_{R}$, where 
$\tau_{R}$ is the period of a full radial oscillation. In all realistic galactic potentials $\Omega_{\phi}<\Omega_{R}<2 \Omega_{\phi}$, and stars therefore make more than one, but less than two radial oscillations per orbit period. For near-circular orbits, $\Omega_{\phi} \rightarrow \Omega_{c}$, and the radial oscillation becomes harmonic about the guiding center, with $\Omega_{R} \rightarrow \kappa$, the Lindblad epicyclic frequency defined through

$$
\kappa^{2}\left(R_{g}\right)=\left(\frac{\partial^{2} \Phi}{\partial R^{2}}\right)_{\left(R_{g}, 0\right)}+\frac{3}{R_{g}}\left(\frac{\partial \Phi}{\partial R}\right)_{\left(R_{g}, 0\right)} .
$$

Motion in the third dimension can also be oscillatory, with a well-defined period $\tau_{z}$. When both the radial and vertical excursions are small, the vertical oscillation is decoupled from the in-plane motion, and has an angular frequency $\nu$ given by

$$
\nu^{2}\left(R_{g}\right)=\left(\frac{\partial^{2} \Phi}{\partial z^{2}}\right)_{\left(R_{g}, 0\right)} .
$$

Naturally, $\Omega_{z} \equiv 2 \pi / \tau_{z} \rightarrow \nu$ in this limit.

In a general static, axisymmetric potential, the motion of most stars can be decomposed into three separate oscillations at the three different frequencies, $\Omega_{R}, \Omega_{\phi}$, and $\Omega_{z}$. Such orbits are described as regular orbits. However, there is a generally small fraction of irregular orbits, whose motion is more complicated and cannot be decomposed into three oscillations and another fraction that are truly chaotic.

In addition to the two classical integrals $E$ and $L_{z}$, regular orbits respect a third, nonclassical integral. It is described as a nonclassical integral because it cannot be expressed as a simple function of the phase-space variables.

\section{Action-angle variables}

The formal clutter that usually accompanies any introduction to action-angle variables makes it hard to grasp what they really are and why they are useful. In an attempt to clarify these points, I here give a brief qualitative discussion, and refer the interested reader to BT08 for a more mathematical, but still not fully rigorous, treatment.

The 2D axisymmetric case, for which there are just two actions, is easiest to visualize and was illustrated already in Fig. 1. The azimuthal action $J_{\phi}$ is simply the angular momentum, which is a measure of the size of the orbit or equivalently the radius of the guiding center (Eq. (6)). The radial action $J_{R}$ is a second conserved quantity that is a measure of the radial extent of the oscillation; thus $J_{R}=$ 0 for a circular orbit and nonzero values can be calculated using Eq. (11). The orbit is uniquely determined by the values of the actions $\left(J_{R}, J_{\phi}\right)$, which are an alternative pair of integrals to $\left(E, L_{z}\right)$.

The doubly periodic motion is described by two angles $w_{R}$ and $w_{\phi}$, which specify respectively the phase of the orbit around its epicycle and the phase of the guiding center around the galaxy center. One major advantage of this apparatus is that each angle variable increases at the constant rate $w_{i}(t)=w_{i}(0)+t \Omega_{i}\left(E, L_{z}\right)$, even though the $(R, \phi)$ coordinates of a star vary nonuniformly with time. $^{2}$

This approach becomes far more valuable when used to describe the 3D motion of a regular orbit, which respects three integrals. Even though one or more integrals cannot be expressed as simple functions of the phase-space variables in either Cartesian or polar coordinate systems, we can fully describe regular 3D motion in an arbitrary smooth potential using three actions that are conserved quantities, i.e. they are a set of integrals. The triplyperiodic motion is described by three angles that again increase at uniform rates. The actions in an axisymmetric potential are $J_{R}$ and $J_{\phi}$, the radial and azimuthal actions as for $2 \mathrm{D}$, and $J_{z}$, which quantifies the up-anddown motion about the midplane. For each regular orbit, the three oscillation frequencies are $\Omega_{i}=d w_{i} / d t=2 \pi / \tau_{i}$ (乌II.C), with $i$ denoting any of the three cylindrical polar coordinates.

Not only do we now have a set of integrals and can describe the motion as the three angles increase in time at uniform rates, but these variables have two further advantages. The actions are the adiabatic invariants when the system is subject to slow changes, and the orbit is not close to a resonance. Finally, a more mathematical advantage is that perturbation theory is greatly simplified when using these variables (e.g. $₫$ III.E).

\section{E. Orbital tori}

Were motion confined to a plane, as for the $2 \mathrm{D}$ orbit shown in Fig. 1, the star would move in the 4D phase space $\left(R, \phi, v_{r}, v_{\phi}\right)$. However, because both $E$ and $L_{z}$ are conserved, the star's motion is confined to a $2 \mathrm{D}$ surface within the $4 \mathrm{D}$ phase space, since both velocity components $v_{\phi}=L_{z} / R$ and $v_{R}=\left\{2[E-\Phi(R)]-\left(L_{z} / R\right)^{2}\right\}^{1 / 2}$ are determined for every value of $R$, except for the sign ambiguity of $v_{R}$.

To see that the motion is confined to the surface a torus, imagine that we add to the rosette orbit shown in the upper panel, a third coordinate that is the star's radial velocity $v_{R}$, which is positive above the page and negative below. As the star moves forward in time from its pericentric distance, say, $v_{R}$ first rises to a maximum height above the page as it crosses $R_{g}$, and then decreases to zero as it reaches its apocentric distance. Then $v_{R}$ changes sign and the inward motion is below the page. As the star moves out and in, it also advances around the galactic center. Thus the motion in the $3 \mathrm{D}$ space

\footnotetext{
2 Lynden-Bell (1962) pointed out that while $w_{i}(0)$ is a constant of the motion, it is a nonisolating integral, and therefore is of no importance to the overall structure of phase space.
} 
of $\left(R, \phi, v_{R}\right)$ is confined to the surface of a torus in that space. It is impossible to visualize a fourth dimension, but while the speed $v_{\phi}$ around the torus varies with $R$, it does so within a restricted range that does not alter the topology.

In $3 \mathrm{D}$, stars move in a $6 \mathrm{D}$ phase space, and every conserved quantity, or isolating integral, confines its motion to a hyper-surface of one lower dimension. The regular orbit of a star that possesses three integrals is confined to the surface of a 3D hyper-torus in the $6 \mathrm{D}$ phase space, and again the motion within each dimension of the hypertorus is an independent oscillation.

Fewer quantities are conserved for chaotic orbits, whose motion cannot be decomposed into three independent oscillations. For example, a star that moves chaotically is not confined to a $3 \mathrm{D}$ surface, but explores a $5 \mathrm{D}$ space if only $E$ is conserved, which is typical in a nonaxisymmetric potential.

All three actions are quantities having the dimensions of angular momentum, and each is defined as the crosssectional area of the appropriate slice through the star's orbit torus in $6 \mathrm{D}$ phase space, i.e.

$$
J_{i} \equiv \frac{1}{2 \pi} \oint \dot{x}_{i} d x_{i},
$$

where $i$ labels a particular coordinate and the integral is around one complete loop in this slice through the torus. In an axisymmetric potential, $J_{\phi} \equiv(2 \pi)^{-1} \int_{0}^{2 \pi} v_{\phi} R d \phi$ and, since the integrand $R v_{\phi}=L_{z}$ is a constant, we have $J_{\phi} \equiv L_{z}$, but Eq. (9) generally must be evaluated numerically for the other actions.

Since stars oscillate at differing frequencies in each of the three coordinate directions, one way to estimate the $i$-th action is to integrate the orbit and measure the area inside the closed curve delineated by the locus of points, or consequents, as the star crosses the 2D surface $\left(x_{i}, \dot{x}_{i}\right)$, known as the surface of section. McMillan \& Binney (2008) described a superior method of "torus fitting" that yields all three actions simultaneously in an arbitrary potential.

For those who find torus fitting intimidating, useful approximations can be obtained more easily for disk star orbits. We first assume axial symmetry, so that $J_{\phi}=L_{z}$, and write the energy of a star as

$$
E=\frac{1}{2}\left(\dot{R}^{2}+\dot{z}^{2}\right)+\Phi_{\mathrm{eff}}(R, z),
$$

with the effective potential being $\Phi_{\text {eff }} \equiv \Phi(R, z)+$ $L_{z}^{2} /\left(2 R^{2}\right)$. The approximation is to assume that the radial and vertical oscillations are decoupled, and that the radial action can be estimated from motion in the midplane as

$$
\left.J_{R} \simeq \frac{1}{\pi} \int_{R_{p}}^{R_{a}}\left\{2\left[E-\Phi_{\mathrm{eff}}(R, 0)\right]\right\}^{1 / 2} d R\right|_{z=0} .
$$

The integration limits are the pericentric and apocentric values of $R$ in the midplane, where the argument of the square root is zero. This integral is for half the radial period, and we drop the factor 2 in the denominator because the return half makes an equal contribution. Similarly,

$$
\left.J_{z} \simeq \frac{1}{\pi} \int_{z_{\min }}^{z_{\max }}\left\{2\left[E-\Phi_{\mathrm{eff}}\left(R_{g}, z\right)\right]\right\}^{1 / 2} d z\right|_{R=R_{g}} .
$$

These equations are exact for stars lacking vertical or radial oscillation, respectively, but in general they are slight overestimates since they assume that a star moving in 3D explores the full extent of the region that is energetically accessible, which is not quite the case when the orbit is integrated.

The epicyclic approximation for small-amplitude excursions assumes that both the radial and vertical oscillations are harmonic. If this is valid, $J_{R \text {,epi }}=$ $\kappa a^{2} / 2$, with $a$ being the radial excursion of the star (Lynden-Bell \& Kalnais, 1972) and $J_{z, \text { epi }}=\nu z_{\max }^{2} / 2$. Since most stars in a disk have vertical excursions that take them outside the harmonic region of the disk potential well, $J_{z, \text { epi }}$ should be avoided in favor of Eq. (12), which is readily evaluated.

\section{F. Distribution function}

The density of stars in the $6 \mathrm{D}$ phase space of position and velocity is given by a distribution function, $f$, hereafter DF. The DF for an equilibrium stellar system must be a function of the integrals only (Jeans theorem). The set of actions is a possible set of integrals, and the density of regular orbits could be written as $f\left(J_{1}, J_{2}, J_{3}\right)$, but formally only if every possible orbit respects three integrals and there are no irregular or chaotic orbits.

For axisymmetric systems, if there were no third integral, the DF would be a function of the two classical integrals, $E$ and $L_{z}$ only. If this were true, the ratio of the second moments of the radial and vertical velocity components,

$$
\frac{\left\langle v_{R}^{2}\right\rangle}{\left\langle v_{z}^{2}\right\rangle}=\frac{\int f\left(E, L_{z}\right) \dot{R}^{2} d v^{3}}{\int f\left(E, L_{z}\right) \dot{z}^{2} d v^{3}}=1,
$$

as both $\dot{R}$ and $\dot{z}$ enter equally in $E$ (Eq. 10). Since we observe that $\sigma_{R} \neq \sigma_{z}$, we conclude that large parts of phase space of disks are regular and the effective DF must depend upon three integrals.

A few analytic expressions for DFs are known for 2D disks, but because the possible third integral is not a simple function of the phase-space coordinates, the development of analytic three-integral DFs for realistic flattened disks (e.g. Binney, 2010) is much more difficult.

\section{G. Nonaxisymmetric disturbances}

Consider the potential of a small-amplitude disturbance in the $z=0$ midplane that is the real part of

$$
\Phi_{1}(R, \phi, t)=\Psi(R) e^{i(m \phi-\omega t)} .
$$


This potential has the following properties: it varies sinusoidally with the azimuthal coordinate $\phi$, it is $m$-fold rotationally symmetric (e.g., $m=2$ for a bi-symmetric spiral or bar), it rotates about the $z$-axis at the angular rate $\Omega_{p}=\Re(\omega) / m$ which is described as the pattern speed, and grows exponentially at the rate $\gamma=\Im(\omega)$. The complex function $\Psi(R)$ describes the radial variation of the amplitude and phase of the perturbation.

The density distribution that gives rise to this disturbance potential is not easily computed. Generally, Poisson's equation requires the potential spiral to be less tightly wound than the density spiral, and the phase relation between the density and potential therefore varies systematically with radius. The density and potential are in phase when the tight-winding (or WKB) approximation is employed, but spirals in galaxies are sufficiently open that this approximation gives only a rough guide to the dynamics of real spirals.

\section{H. Resonances}

Stars orbiting in the midplane of an almost axisymmetric galaxy are in resonance with a weak nonaxisymmetric disturbance of the form (14) when

$$
\Omega_{p}=\Omega_{\phi}+\frac{l}{m} \Omega_{R}
$$

The unperturbed orbit frequencies of the stars are defined in $\$ 11 . \mathrm{C}$ and $l$ is a signed integer. Equation (15) is satisfied for $l=0$ when the guiding center of a star rotates synchronously with the disturbance, which is described as the corotation resonance. When $l= \pm 1$, Eq. (15) defines the locations of the Lindblad resonances, which arise because the Doppler shifted frequency at which the star encounters the wave $m\left|\Omega_{\phi}-\Omega_{p}\right|$ is equal to its unforced frequency of radial oscillation $\Omega_{R}$, or $\kappa$ for nearly circular orbits. Interior to corotation, the stars overtake the wave, and $l=-1$ at the inner Lindblad resonance (ILR). Outside corotation, the stars are overtaken by the wave, and the outer Lindblad resonance (OLR) occurs where $l=+1$. Resonances for larger values of $|l|$, if they occur at all, are generally of little dynamical interest, since spiral patterns can exist only between the Lindblad resonances; a steady perturbing potential does not elicit a supporting response from the stars outside this radial range.

Ultraharmonic resonances arise where Eq. (15) is satisfied for $l= \pm 1$ and $m$ replaced by $2 m$. At these resonances, which are closer to corotation than are the Lindblad resonances, the star completes two radial oscillations as it moves between wave-crests. Yet higher-order resonances exist for larger integral numbers of radial oscillations; they are located still closer to corotation as stars drift ever more slowly relative to the pattern. Ultraharmonic resonances are dynamically unimportant in linear perturbation theory, but their nonlinear generalizations do play a role in finite-amplitude perturbations, especially bars $(\sqrt{\mathrm{V}})$.
Vertical resonances will occur where the Doppler shifted frequency

$$
m\left(\Omega_{p}-\Omega_{\phi}\right)=n \Omega_{z},
$$

with $n$ being a signed integer; the $n=0$ case (corotation) is of no special significance for vertical motion. In the epicycle approximation, $\Omega_{z} \rightarrow \nu$, which is a higher frequency in the massive part of the disk than is $\kappa$. Therefore, the $n= \pm 1$ vertical resonances are farther from corotation than are the Lindblad resonances. In linear perturbation theory, spiral perturbations do not extend beyond the Lindblad resonances, making these vertical resonances uninteresting because the perturbation potential is tiny there. However, it should be noted that the effective vertical frequency $\Omega_{z} \equiv 2 \pi / \tau_{z}$ can be much smaller than $\nu$ for stars with vertical excursions extending well outside the region where the potential is approximately harmonic, and such stars could, in principle, experience a vertical resonance.

Linear perturbation theory holds even at resonances for small-amplitude disturbances that grow exponentially, for then the resonances have a Lorentzian width determined by the growth rate. However, it breaks down for stars in resonance with a steady, or slowly growing, perturbation. Stars can be trapped by the resonance, and the size of the trapped region in phase space increases with the amplitude of the perturbing potential.

\section{Local stability}

The problem of computing the gravitational potential of an arbitrary spiral disturbance is one reason that the global normal modes of a stellar disk cannot be computed in a straightforward manner (Kalnajs, 1977); (Jalali, 2007, BT08). While a WKB (local) approach, in which the local spatial variation of the disturbance can be approximated as part of a plane wave, is generally a poor approximation, results obtained using it do give some indication of the global behavior.

Toomre (1964) used this approximation to show that axisymmetric oscillations in a razor-thin disk of surface density $\Sigma$ are stabilized by rotation on scales

$$
\lambda>\lambda_{\text {crit }}=\frac{4 \pi^{2} G \Sigma}{\kappa^{2}},
$$

where $\kappa$ is the epicyclic frequency defined in Eq. (7). In the complete absence of random motion, a disk is unstable to gravitational clumping into rings on all scales smaller than $\lambda_{\text {crit. }}$. Physically, $\lambda_{\text {crit }}$ decreases with increasing $\kappa$ because stars are held more tightly to their guiding center radii. The value of $\lambda_{\text {crit }} \approx 6 \mathrm{kpc}$ in the solar neighborhood, or three fourths of the Sun's distance from the Galactic center, indicating that the WKB approximation is indeed stretched.

Random motions of the stars prevent gravitational instabilities when the disturbance disperses more rapidly 
than it grows. The tendency of random motions to provide stability on small scales while rotation suppresses instability on large scales, led Toomre to the following celebrated local stability criterion: if the stellar radial velocities have a Gaussian distribution with spread $\sigma_{R}$, then the system is axisymmetrically stable on all scales if

$$
Q \equiv \frac{\sigma_{R}}{\sigma_{R, \text { crit }}}>1, \quad \text { where } \quad \sigma_{R, \text { crit }}=\frac{3.36 G \Sigma}{\kappa} .
$$

Adopting solar neighborhood values, we find $\sigma_{R, \text { crit }} \approx$ $20 \mathrm{~km} \mathrm{~s}^{-1}$. In a razor-thin, isothermal gas disk $\sigma_{R}$ is replaced by the sound speed $c_{s}$ and the constant 3.36 is replaced by $\pi$. The constant is also slightly reduced in finitely thick disks since the destabilizing gravitational forces are diluted by the vertical spread of matter (e.g. Romeo, 1992). The local stability of combined gas and stellar disks was calculated by Rafikov (2001), while Romeo \& Wiegert (2011) offer an approximate formula for $Q$ in thickened two-component disks. Global axisymmetric stability may be guaranteed if the disk is locally stable everywhere (Kalnajs, 1976).

It cannot be emphasized too strongly that criterion (18) is for local axisymmetric stability only, and that disks that meet this criterion can still be unstable to nonaxisymmetric modes. In fact, no general criterion for nonaxisymmetric stability is known.

Local nonaxisymmetric stability was investigated by Goldreich \& Lynden-Bell (1965) and by Julian \& Toomre (1966), who independently discovered the process of swing-amplification. Figure 2, from a global calculation due to Toomre (1981), illustrates the fate of an arbitrary input leading spiral inserted by hand and given a pattern speed so that it is localized near the ILR. In this linearly stable, $Q=1.5$ disk, the disturbance initially propagates away from the ILR and unwinds due to the differential rotation until it "swings" from leading to trailing. The disturbance is amplified by a large factor during this period when it is least wound because rotational support, which is a critical part of axisymmetric stability, is compromised briefly. The disturbance propagates radially at the group velocity (Toomre, 1969), which is away from corotation for trailing waves, and the inner part returns toward the ILR. The part of the disturbance outside corotation fades quickly as it spreads over a wider area, while the opposite behavior affects the inner part until it is gradually absorbed by wave-particle interactions as it approaches the resonance. Thus the whole episode is a transient response that, to first order, causes no lasting change to the disk, although there are second order changes.

The amplification of a wave packet at corotation can be calculated in a variety of local approximations (Toomre, 1981), while Drury (1980) computed the relationship between a continuous wave train incident on corotation and the super-reflected and transmitted waves. In the notation of Julian \& Toomre (1966), the most important

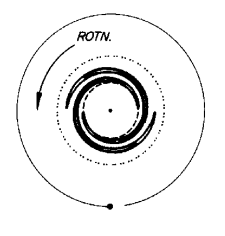

1
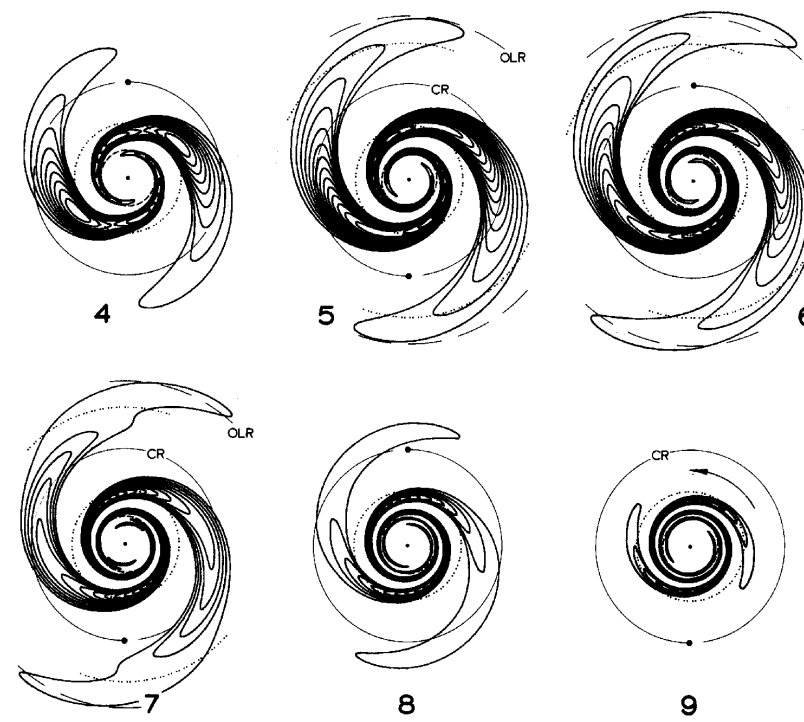

FIG. 2 The time evolution of an input leading wave packet in the half-mass Mestel disk. The unit of time is half a circular orbit period at the radius marked corotation. From Toomre 1981.

parameter is

$$
X \equiv \frac{\lambda_{y}}{\lambda_{\text {crit }}}=\frac{2 \pi R_{\mathrm{CR}}}{m} \frac{\kappa^{2}}{4 \pi^{2} G \Sigma}
$$

where $\lambda_{y}$ is the wavelength of the disturbance with angular periodicity $m$ around the corotation circle of radius $R_{\mathrm{CR}}$. For a flat rotation curve, amplification is significant for $1 \lesssim X \lesssim 3$ and is strongest for an unwrapped wavelength that is about twice $\lambda_{\text {crit }}$. If the rotation curve declines, amplification extends to larger values of $X$ and, conversely, it is confined to smaller $X$ values in rising cases. Of course, the range of $X$ for strong amplification shrinks to zero in the absence of shear (uniform rotation).

The amplification factor also decreases rapidly with increasing $Q$ (Eq. 18). The reflected wave can be 100 times as strong as the incident wave for $X \simeq 2$ and $Q \simeq$ 1.2 , but only a few times greater when $Q \simeq 2$.

Notice that $X \propto(m \Sigma)^{-1}$, implying that for a fixed radius and rotation curve, amplification will be strong for higher $m$ values when the disk surface density $\Sigma$ is low - i.e. we expect bisymmetric spirals in heavy disks and multi-arm spirals in strongly submaximal disks (Sellwood \& Carlberg, 1984). Thus the number of spiral arms in a galaxy could be an indicator of 
the relative contribution of the disk to the total central attraction (Athanassoula et al., 1987). This argument should not be applied to flocculent galaxies (Elmegreen \& Elmegreen, 1982), which have many small arm fragments, where the small spatial scale of the arms probably indicates that the responsive part of the disk is a low-mass component that has become dynamically decoupled from a hotter, underlying stellar disk.

\section{J. Angular momentum changes}

The response of the stars to a weak potential perturbation is most easily calculated in action-angle variables (Lvnden-Bell \& Kalnajs, 1972); (Dekker, 1976); (Carlberg \& Sellwood, 1985); (Binney \& Lacey, 1988). Lynden-Bell \& Kalnajs (1972) showed that the first order changes in the angular momenta of stars average to zero everywhere. However, to second order, the rate of change in angular momentum of a group of stars is

$$
\frac{d L_{\mathrm{tot}, 2}}{d t}=4 \pi^{2} \gamma e^{2 \gamma t} \sum_{\boldsymbol{m}} m \int \boldsymbol{m} \frac{\partial f}{\partial \boldsymbol{J}} \frac{\left|\Phi_{1, \boldsymbol{m}}^{2}\right|}{|\boldsymbol{m} \cdot \boldsymbol{\Omega}-\omega|^{2}} d \boldsymbol{J},
$$

plus a boundary term. Here the ranges of integration are unperturbed angular momenta $L_{z, 1} \leq J_{\phi} \leq L_{z, 2}$ and radial action $0 \leq J_{R} \leq \infty$. The $\Phi_{1, \boldsymbol{m}}$ are Fourier coefficients of the perturbing potential $\Phi_{1}$ (Eq. 14), $\boldsymbol{m}=$ $(l, m), \boldsymbol{J}=\left(J_{R}, J_{\phi}\right)$, etc. Resonant denominators arise in Eq. (20) where $\boldsymbol{m} \cdot \boldsymbol{\Omega}=\Re(\omega)$ (the same condition as Eq. 15), which pick out important locations in phase space where substantial changes take place. Note that the magnitude of each $\boldsymbol{m}$-term depends on the gradient of the DF with rrespect to the actions at that resonance; thus the net change depends on the imbalance between those stars that lose on one side of the resonance compared with those that gain on the other side.

Generally, we expect $f$ to be a decreasing function of all the actions in any reasonable galaxy; i.e. for a given $L_{z}$, there are more stars with small $J_{R}$ and $J_{z}$ and $f$ falls off steeply with increasing values of either of these actions. Also the density of disk stars generally rises toward the center, and therefore $f$ rises with decreasing $J_{\phi} \equiv L_{z}$, which is usually the shallowest of the three gradients.

A self-excited spiral involves no external torque, and this expression must therefore integrate to zero over the whole disk. However, Lynden-Bell \& Kalna js (1972) showed that the mean angular momenta of stars inward of corotation is lowered, and those outward are raised, by the growth of the disturbance. This feature allows a mode to extract energy from the gravitational potential well of the galaxy, enabling it to grow. Unfortunately, these angular momentum changes cannot be equated to the gravity torque between the misaligned density and potential because angular momentum can also be transported by a Reynolds-like advective stress (dubbed "lorry transport" by Lynden-Bell \& Kalna js, 1972). The Reynolds stress is probably of minor importance in the vigorously growing modes that galaxies seem to support, but would be significant were quasi-steady spiral modes important.

\section{K. Gas}

The stars of a galaxy move on ballistic orbits that are affected only by gravitational forces. The fraction of the total baryonic mass contained in gas is generally less than $10 \%$ in large disk galaxies today. Over time, gas is converted into stars, but is replenished partly by returned material as massive stars end their lives, and also by on-going infall in spiral galaxies. The interstellar gas is collected into clouds, the diffuse ones being composed largely of neutral atomic hydrogen and helium with a sound speed $c_{s} \sim 1.3 \mathrm{~km} \mathrm{~s}^{-1}$, while dense molecular gas clouds are colder with $c_{s} \lesssim 0.5 \mathrm{~km} \mathrm{~s}^{-1}$.

Typical orbital speeds in galaxies are $100-200 \mathrm{~km} / \mathrm{s}$, while typical velocity spreads of clouds about the mean orbital motion appear to have a lower bound of some $6-8 \mathrm{~km} \mathrm{~s}^{-1}$, rising to twice this value in the bright, star-forming parts of disk galaxies. This supersonic turbulence (see Scalo \& Elmegreen, 2004, for a review) is maintained by a variety mechanisms, the most important of which is mechanical energy input through supernovae and, to a lesser extent, stellar winds (streams of particles accelerated from the surfaces of massive stars). When many massive stars are born at similar times in an exceptional burst of star formation, the ensuing rapid succession of supernovae can create a galactic wind that drives some of the gas out of the disk plane and perhaps, in the cases of small galaxies with shallow potential wells or young galaxies with high rates of star formation, right out of the galaxy. Large-scale dynamical phenomena, such as spiral activity, tidal interactions, and gas infall are other sources of turbulence.

The medium is also stirred by the magnetorotational instability of weakly-magnetized differentially rotating fluid disks (Balbus \& Hawley, 1998); (Sellwood \& Balbus, 1999), which maintains a lower level of trans-Alfvénic turbulence in parts of disks that have few young stars, and correspondingly few supernovae, where the dispersion remains about $6 \mathrm{~km} \mathrm{~s}^{-1}$ (e.g. Dickey et al., 1990); (Tamburro et al., 2009).

High spatial resolution simulations of this medium in small volumes (Stone et al., 1998); (MacLow, 1999) suggest that the magnetic field plays at most a secondary role in the dynamics of the gas clouds, which have a small filling factor. Collisions between clouds are highly supersonic and therefore strongly dissipative, with the thermal energy being radiated efficiently.

This complex medium is radiatively heated by stars to an extent that varies strongly with the proximity to clusters of hot young stars. It is also cooled radiatively through processes such as thermal bremsstrahlung, recombination lines from excited electronic states at rates that depend strongly on the fraction of heavy elements, 
various molecular rotational and vibrational transitions, and thermal emission from dust.

All these processes are intensely localized on spatial scales that are tiny compared with the overall size of a galaxy, and therefore well below the resolution limits of most simulations that attempt to model the formation and evolution of galaxies (see $\S ₫$ II.A and V.E).

However, despite the complicated microphysics of this heated, cooled, magnetized, and stirred multiphase medium, the crucial point is that turbulence cascades down to small scales where it is dissipated. Dissipation of random energy is the most important role of gas in the overall dynamics of the star plus gas disk. Galaxies lacking even a small fraction of mass in gas barely evolve. I emphasize the role of gas in appropriate places in this review.

\section{Gravity softening in simulations}

Computer simulations are powerful tools that have proved indispensible for unraveling the sometimes mystifying behavior of disk galaxies. Yet even with presentday computational power, simulations cannot routinely employ as many particles as there are stars in a galaxy. Thus some degree of smoothing of the mass distribution is needed, which also prevents strong accelerations during close encounters between the particles that would otherwise require adaptive time steps. Smoothing can be introduced directly through "softening" the interparticle force law at short range, or indirectly through the use of a grid, which similarly weakens the forces between particles on scales of the cell size (see the appendix of Sellwood \& Merritt, 1994). A third, but less general, method of smoothing is to determine forces from an expansion in some set of basis functions that is truncated at low order.

Note that shot noise from the particle distribution remains the most important limitation of simulations. The contribution of distant particles to the relaxation rate is unaffected by softening, which smooths fluctuations on only the smallest scales, and changes nothing in the formulae for relaxation but the value of the denominator of $\Lambda$ in the Coulomb logarithm (\$.B). Noisedriven density variations on larger scales can also excite non-negligible collective responses (Sellwood, 1983); (Toomre \& Kalna is, 1991); (Weinberg, 1998).

\section{2D simulations}

Simulating galaxy disks with the motion of particles confined to a plane has the obvious advantage of reduced computational cost over fully 3D simulations. The most appropriate gravity softening rule for $2 \mathrm{D}$ simulations is the Plummer law, for which the potential at distance $d$ from a point mass is:

$$
\Phi_{\mathrm{P}}=-G \mu\left(d^{2}+\epsilon^{2}\right)^{-1 / 2}
$$

where $\epsilon$ is the gravitational softening length.

An advantage of the Plummer softening rule for this application is that it provides an approximate allowance for disk thickness, as follows. Convolution of Eq. (21) with the mass distribution of a razor-thin disk yields the exact Newtonian field in a plane offset by a vertical distance $\epsilon$ from that containing the mass. In real finitely thick galaxy disks, the field everywhere is the sum of the Newtonian fields of the various mass elements spread in layers parallel to the midplane. The Newtonian forces experienced by the stars are therefore weaker than if the mass distribution were razor thin. Thus the value chosen for $\epsilon$ in a $2 \mathrm{D}$ simulation should be closely related to the finite thickness of the disk (Romeo, 1998).

Note that gravity softening weakens nonaxisymmetric instabilities (Sellwood, 1983). Since the Newtonian potential of an arbitrary razor-thin mass distribution can be determined by expansion in Bessel functions (BT08 $\S 2.6 .2)$, the potential of each radial wavenumber, $k$, of the expansion decays away from the plane as $\exp (-|k z|)$. Further, since softened gravity is equivalent to sampling the field of a $2 \mathrm{D}$ mass sheet in a plane offset vertically by a distance $\epsilon$, the disturbance potential of each term is weaker by the factor $\exp (-|k| \epsilon)$. Hence instabilities are less vigorous. However, this weakening is physically realistic because softening provides an approximate allowance for the real finite thickness of galaxy disks as explained above.

To estimate the time for peculiar velocities to be randomized by encounters in 2D simulations, we replace Eq. (3) with

$$
\tau_{\text {relax }}=\frac{\beta^{3} v^{3} \epsilon}{8 G^{2} \mu^{2} n},
$$

where $n$ is now the number of particles per unit area, $\epsilon=b_{\min }$ and we assumed $b_{\max }^{-1} \ll b_{\min }^{-1}$. This formula, without the $\beta^{3}$ factor, was already given by Hohl (1973). Setting $N=\pi R_{e}^{2} n$, with $R_{e}$ being the half-mass radius of the disk, we find for $2 \mathrm{D}$ disks

$$
\tau_{\text {relax }} \approx \frac{\beta^{3} \pi}{8} \frac{\epsilon}{R_{e}} N \tau_{\text {dyn }} .
$$

This time is estimated for particles that interact with the forces derived from the potential of Eq. (21).

An advantage of computing forces through a cylindrical polar grid is that one can further smooth the mass distribution by restricting the sectoral harmonics $m$ that contribute to the forces acting on each particle. The effect of restricting force terms to include only the range $0 \leq m \leq m_{\max }$ is to replace each point particle by an azimuthally extended mass, providing some additional smoothing of the density distribution.

\section{Simulations of thickened disks}

In 3D simulations, the Plummer softening law (Eq. 21) has the undesirable property of weakening the interpar- 
ticle force at all distances from the source particle, and a softening kernel that weakens forces only to a finite-range is greatly preferred. All that is needed for a serviceable softening kernel is that it should join smoothly to the Newtonian law at some distance $\epsilon$ and yield an interparticle force for $d<\epsilon$ that smoothly approaches zero as $d \rightarrow 0$. The precise form of the force at short-range should not matter because forces in a collisionless fluid are dominated by the distant mass distribution. Thus, if the behavior of the $N$-body system is to mimic that of a galaxy, its evolution should be insensitive to the adopted force law at short-range. Put another way, if the choice of the softening kernel affects the behavior, then the simulation is not collisionless.

Of its very nature, gravity softening limits the sharpness of forces that arise from steep density gradients. While the in-plane density distribution of galaxy disks varies on spatial scales that greatly exceed the values of $\epsilon$ generally adopted, the disk mass is strongly confined to a plane. Unless the value of $\epsilon \ll z_{0}$, the restoring force to the midplane will be weakened significantly, which has adverse consequences for the correct representation of buckling instabilities (see §V.D).

In quasispherical mass distributions, the relaxation rate is given by Eq. (4), with $b_{\min }=\epsilon$ in the Coulomb logarithm. Following the discussion in $\llbracket . B 2$, we replace Eq. (4) for disks having a characteristic thickness, $z_{0}$, with

$$
\tau_{\text {relax }} \approx \frac{\beta^{3} \ln \left(R_{e} / b_{\min }\right)}{8 \ln \left(z_{0} / \epsilon\right)} \frac{z_{0}}{R_{e}} N \tau_{\text {dyn }},
$$

where the factor $\beta^{3}$ is appropriate for the peculiar velocities to be randomized by encounters. For typical disk values of $\beta \sim 0.1$ and $z_{0} / R_{e} \sim 0.1$, this time is almost $\sim 10^{4}$ times shorter than for quasispherical, pressuresupported systems with the same $N$ (see also Sellwood, 2013b).

\section{TRANSIENT SPIRAL MODES}

The large majority of disk galaxies manifest beautiful spiral patterns of some form or other. The patterns are sometimes quite coherent and symmetric, which are described as "grand design" spirals, or the overall pattern can have little clear symmetry with individual pieces of spiral arm being hard to trace over long distances because they bifurcate or fade. The more coherent patterns are often seen in galaxies that are barred or have recently suffered a tidal interaction with a passing companion galaxy (Kormendy \& Norman, 1979); (Kendall et al., 2011). However, the ubiquity of the spiral phenomenon, and the fact that similar patterns develop in simulations of stellar disks even when the influences of bars and companions are excluded (Sellwood \& Carlberg, 1984); (Roškar et al., 2008a); (Fuiii et al., 2011); (Wada et al., 2011), suggest that spirals in galaxies can also be selfexcited.
Spirals are important to secular evolution because they transport angular momentum to a limited extent (see (III.E), scatter stars at Lindblad resonances, which increases random motion, cause radial mixing, and smooth rotation curves. I discuss each of these processes in turn.

\section{A. Origin and Recurrence}

The precise mechanism that causes spiral patterns to develop is not fully understood and a thorough survey of the various ideas would require too long a digression here (see Sellwood, 2013a, for a recent review). There is general agreement among theorists that spirals are gravitationally driven density waves in the stellar disk, for which there is substantial body of supporting observational evidence, both photometric (Schweizer, 1976); (Gnedin et al., 1996); (Grosbøl et al., 2004); (Zibetti et al., 2009) and kinematic (Visser, 1978); (Chemin et al., 2006); (Shetty et al., 2007). While the idea that spiral patterns could be long-lived, or quasisteady, features has been advocated for some time (e.g. Bertin \& Lin, 1996), it seems increasingly certain that an individual spiral pattern does not persist for more than a few disk rotations (Sellwood, 2011). The supporting evidence has to be indirect, since we cannot observe the time evolution of real galaxies, and is based on the behavior in simulations, which has not changed as their quality has improved, and is supported by the arguments developed below that disk evolution makes more sense when spirals are short-lived.

Most simulations manifest spiral patterns whose appearance changes on time scales of less than one rotation of the disk. However, power spectrum analysis (Sellwood, 1989a) showed that the extreme variability of the spirals reported by Sellwood \& Carlberg (1984) was caused by the superposition of a few underlying longer-lived waves, as has subsequently been found by others (e.g. Roškar et al., 2012); (Grand et al., 2012); (Minchev et al., 2012a).

Wada et al. (2011), Grand et al. (2012), Baba et al. (2013), and Roca-Fàbrega et al. (2013) reported that spirals in their simulations are almost material features that wind up over time according to the local shear rate of the disk. This behavior could also result from the superposition of multiple waves, as illustrated in Fig. 3] which shows the visual appearance of the combined density of two separate fixed-amplitude waves that rotate at different angular frequencies. As long as the inner wave has the higher angular speed, the combined density has most of the properties reported by these authors.

The title of this section contains the word "modes" to distinguish what is meant from the "transient spiral" illustrated in Fig. 2, which shows the time evolution of the vigorous disk response to a particularly provoking peturbation. That transient response is neither an instability, because it does not grow indefinitely, nor a mode, because it does not have a fixed shape. A mode, by contrast, is a 


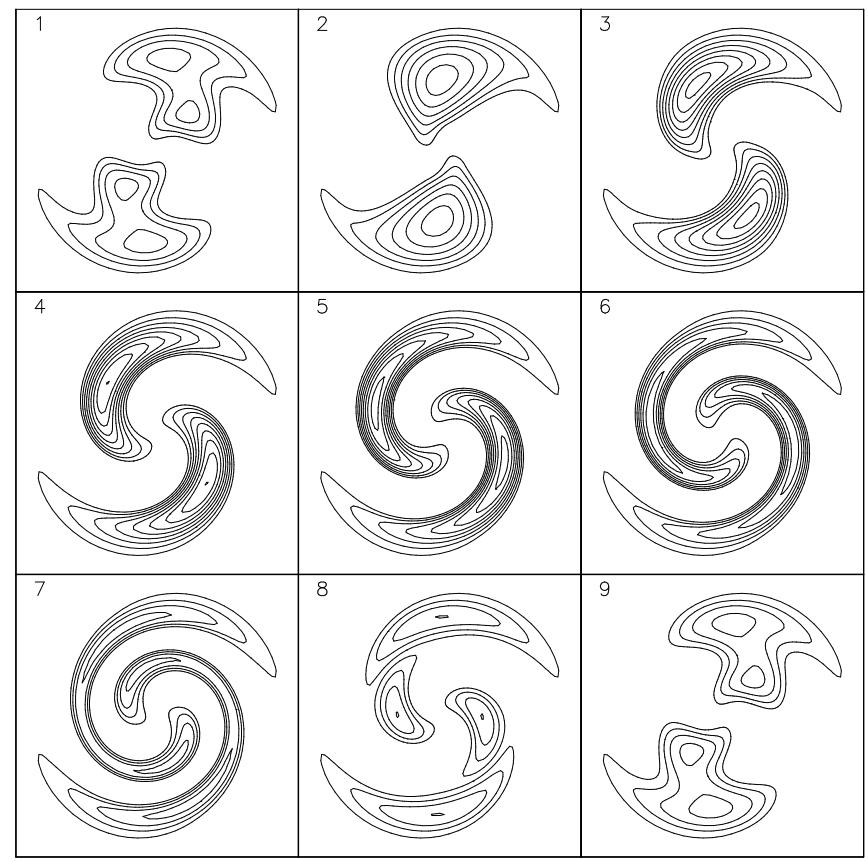

FIG. 3 Contours of the combined positive overdensity that results from the superposition of two open spiral patterns that each has constant amplitude and rotates at a steady, but different, rate. This is purely a set of drawings, with no underlying dynamics. The numbered sequence illustrates one full beat period, and is shown in a frame that rotates such that the outer wave does not appear to move. Notice that the largest net overdensities occur when the spiral is moderately wrapped. An animation is available at http://www.physics.rutgers.edu/ sellwood/spirals.html

standing wave oscillation of the system having a constant shape and frequency.

A few authors have risen to the challenge of solving for the normal modes of a smooth stellar disk with random motion. They found vigorously unstable bar-forming (Kalnajs, 1972, 1978); (Jalali, 2007) and lop-sided (Zang, 1976), (Evans \& Read, 1998) modes. However, when these instabilities are avoided, perhaps by embedding the disk in a halo (see $\mathrm{V.B}$ ), studies of smooth disks generally do not reveal milder spiral modes (Toomre, 1981). An exception was the study by Bertin et al. (1989), who found slowly-growing bi-symmetric spiral modes in lowmass, cool disks. But Sellwood (2011) showed that their adopted disk models would not survive, since they were subject to more vigorous multiarm instabilities that caused the background state of the disk to heat quickly. Thus these normal mode analyses have been useful to understand global disk stability, but have not yielded any promising spiral-causing modes.

However, the waves that underlie the rapidly changing patterns in the snapshots and movies from simulations do appear to be genuine modes of the disk. Each has a fixed shape and well-defined pattern speed, and grows and decays on timescales of a few disk rotations. As they are not truly long-lived, they are best described as "transient spiral modes."

Each spiral mode in the simulations is a vigorously growing instability that saturates at an overdensity of some $20 \%-30 \%$ relative to the local unperturbed disk density. After it saturates it fades just about as quickly as it grew, with all the wave action that had been extracted from the particles during its growth being carried radially away from corotation at the group velocity (Toomre, 1969). Wave action is finally absorbed at the Lindblad resonances ( (II.H), where wave-particle interactions occur (Lynden-Bell \& Kalnajs, 1972); (Mark, 1974). The changes to the underlying disk caused by the scattering of stars at these Lindblad resonances seed conditions for a new instability to develop Sellwood (2012), and thus the cycle recurs. These instabilities were missed by the authors of the above-cited stability analyses because they studied the modes of an assumed smooth, featureless disk. Sellwood (2010) found some evidence in the velocities of stars in the solar neighborhood to support this picture.

D'Onghia et al. (2013) introduced a collection of heavy, co-orbiting particles into their large- $N$ simulations of low-mass disks, which seeded spiral responses. In one experiment, they introduced a single heavy perturber and removed it again after some evolution; they found that spiral activity continued, which they attributed to additional responses to the fluctuations caused by responses to the earlier forcing particle, in a bootstrap fashion, that they described as "nonlinear" effects, although it was unclear that the behavior they observed depended in any way on the amplitudes of the disturbances. It is also possible to regard their result as the superposition of multiple spiral modes of the underlying disk, which were triggered at moderate amplitude by the original perturbing heavy particle. Whatever the correct explanation, they concurred with Sellwood (2012) that spiral activity at one instant is directly influenced by the immediately preceding activity.

In the absence of dissipation, the recurring spirals drive up the level of random motion in the disk (see $₫$ II.B.2. As $Q$ rises, the disk becomes ever less able to support collective oscillations, and activity weakens on a time scale of perhaps ten disk rotations (Sellwood \& Carlberg, 1984). ${ }^{3}$ At this point the minor gas component (@I.K) takes on a dynamically important role; while dense clouds of gas are accelerated by the spirals in the same manner as are the stars, they are able to dissipate random motion quickly through supersonic collisions that allow the excess energy to be radiated away. The gas clouds themselves, and the stars that form within them, therefore constitute a low velocity dispersion compo-

\footnotetext{
3 Fuijii et al. (2011) suggested the time-scale could be longer, but the spirals in their simulations are quite faint at late times. Furthermore, the dominant halo they use results in multiarm spirals that heat the disk more slowly than would patterns of lower $m$ (
} 
nent that is able to maintain the dynamical responsiveness of the combined star-gas disk. Sellwood \& Carlberg (1984) estimated that a birth rate of a few stars per year over the entire disk of a galaxy would be sufficient to sustain spiral activity indefinitely. Subsequent work (Carlberg \& Freedman, 1985); (Toomre, 1990); (Roškar et al., 2008a) on isolated disks, and fully cosmological simulations (e.g. Agertz et al., 2011), seems to confirm that no matter how the dissipation is mimicked, the disk continues to support transient spiral patterns. This behavior provides an attractive explanation for the long-noted (e.g. Oort, 1962) contrast between the striking spirals manifested by galaxies having abundant gas to the featureless appearance of S0 galaxies that have very little gas.

\section{B. Scattering of stars}

It has been clear for over 50 years that older stars in the neighborhood of the Sun have larger velocity spreads than do younger stars (Wielen, 1977); (Nordström et al., 2004). It seems unsatisfying to suppose that older stars were born with larger random velocity components, since it requires us to live at a special time when random motions at birth have just become small, but this suggestion has been advocated (e.g. Kroupa, 2002). Some initial random motion seems likely in the disturbed conditions of disks in the early Universe when the oldest stars formed, but the progressive increase of random motions of disk stars with increasing intermediate ages is generally attributed to scattering processes. Both massive gas clumps (Spitzer \& Schwarzschild, 1953) and spiral patterns (Barbanis \& Woltjer, 1967) are still considered viable as scattering agents.

\section{Solar neighborhood data}

Fig. 4 shows the variation of stellar velocity dispersion components with estimated age, as presented by Holmberg et al. (2009). (The small scatter about the trend among the points for the older stars is somewhat odd.) These results are synthesized from the heroic Geneva-Copenhagen survey (herafter GCS) of $\sim 14000$ F- and G-dwarf stars by Nordström et al. (2004), with repeated radial velocity measurements of all the stars to eliminate binaries, as well as improved stellar parameter and age calibrations (Holmberg et al., 2007), and the revised Hipparcos distances and proper motions (van Leeuwen, 2007). Holmberg et al. (2009) use the usual notation $\sigma_{U}, \sigma_{V}$, and $\sigma_{W}$ for the radial, azimuthal, and vertical velocity spreads as seen from the Sun, where others may use $\sigma_{R}, \sigma_{\phi}$, and $\sigma_{z}$ for the radial, azimuthal and vertical dispersions anywhere. Casagrande et al. (2011) reanalyzed the same sample adding infrared fluxes for about half the stars to obtain new estimates of stellar parameters and ages, and again found the total veloc-
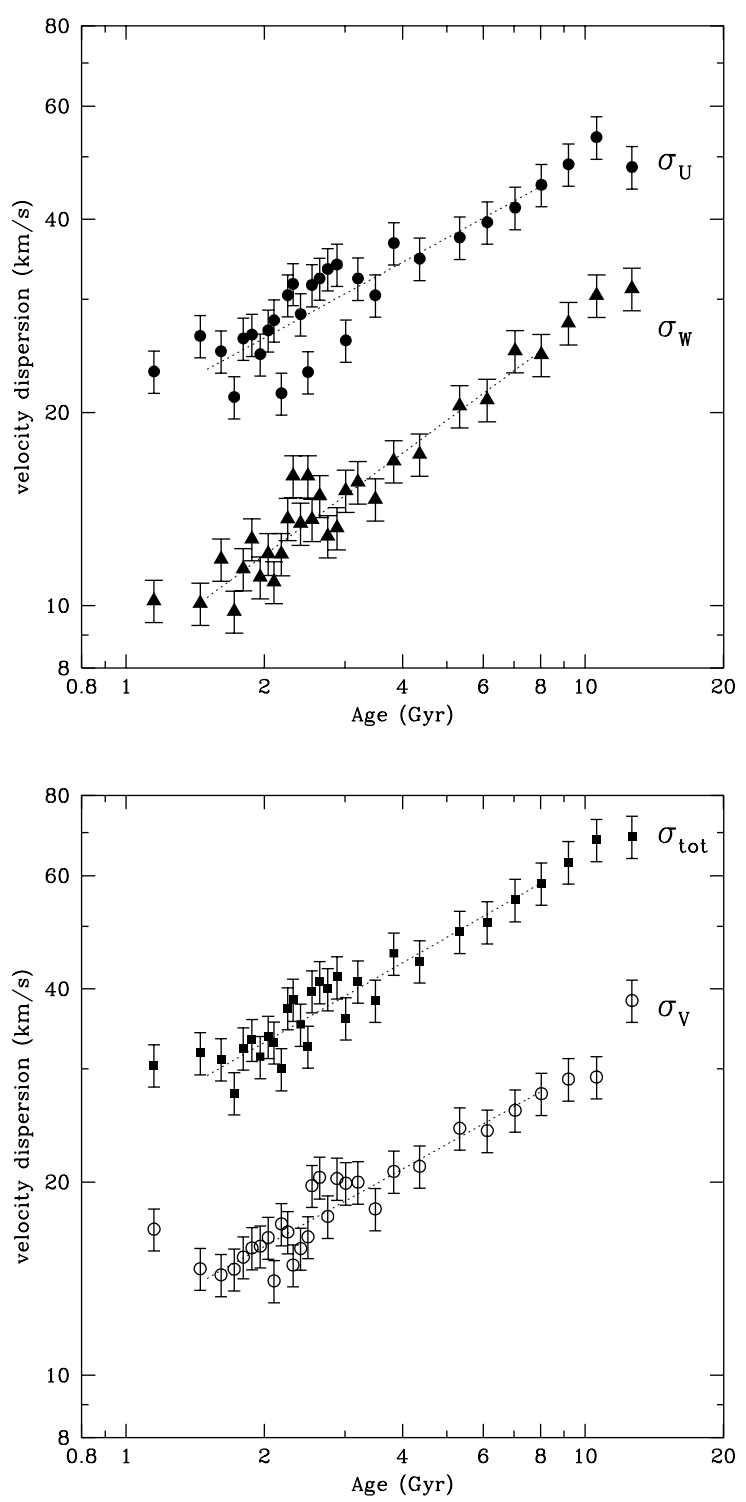

FIG. 4 Estimates of the second moments of stellar velocities from the Geneva-Copenhagen survey. The symbols with error bars show the estimated spreads of the three velocity components in Galactic coordinates: in the upper panel, $U$ is in the radial direction and $W$ in the direction normal to the plane. The spread of $V$ in the azimuthal direction and the total dispersion are shown in the lower panel. The stars were divided by estimates of their ages and the fitted lines ignore the two youngest and the two oldest groups. From Holmberg et al., 2009).

ity dispersion rose steadily with age (their Fig. 17), even when they excluded metal-poor stars.

Assigning ages to individual stars is highly controversial (see Soderblom, 2010, for a review), and the precise trend with age has therefore been the subject of much debate. Reid et al. (2007) suggest that the ages of individual stars assigned by Nordström et al. (2004) and revised 


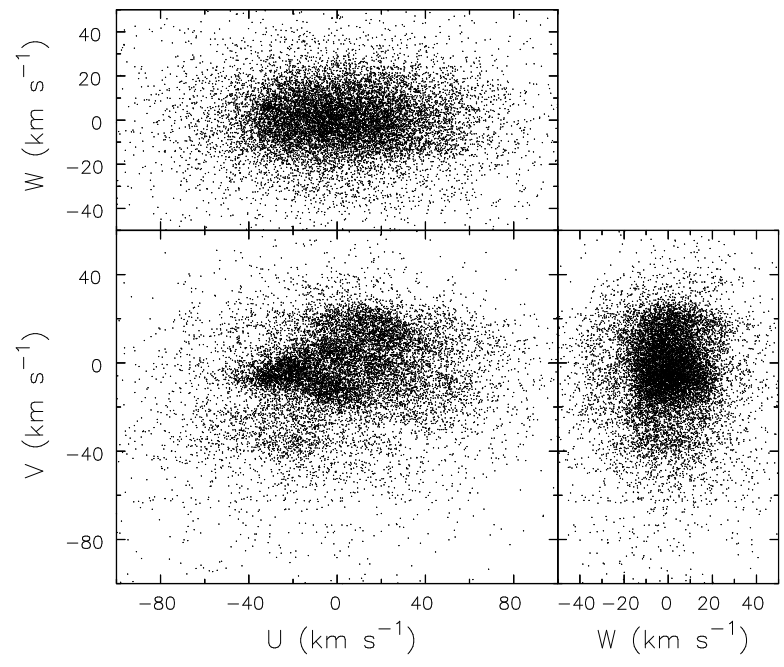

FIG. 5 The stellar velocities from the Geneva-Copenhagen survey (Holmberg et al., 2009), corrected for solar motion $\left(U_{\odot}, V_{\odot}, W_{\odot}\right)=(11.1,12.24,7.25) \mathrm{km} \mathrm{s}^{-1}$ Schönrich et al. $(2010)$. Note the substantial substructure in the $(U, V)$-plane that is not reflected in $W$.

by Holmberg et al. (2007) are compromised by large random errors. Were this the case, then the real trends with age would have to be even steeper than shown in Fig. 4. since large age errors will flatten a trend, as found by Casagrande et al. (2011) when they included stars with more uncertain ages.

On the other hand, Edvardsson et al. (1993) and Quillen \& Garnett (2001) claimed a more nearly constant velocity dispersion with age, except for very old stars that have a larger dispersion. Soubiran et al. (2008) also found an almost flat dispersion with age, although it is unclear whether their exclusion of probable thick disk and halo stars, based at least in part on their kinematics, is affecting the trend. If a constant dispersion with age were correct, and if the small samples of stars used in these studies were drawn from the same population as those selected by Holmberg et al. (2009), then the age errors that give rise to the steady trends seen here in Fig. 4 and in Fig. 17 of Casagrande et al. (2011) would have to correlate with the kinematics. It therefore seems more likely that claims of a flat trend result from large age errors or selection against stars with large peculiar velocities. Furthermore Aumer \& Binney (2009) found that blue main-sequence stars, which must be young, have much smaller random motions than do red mainsequence stars, which can have a wide range of ages. Using color as a proxy for age assumes a well-behaved starformation rate in the disk, which has probably declined slowly over time (e.g. Fraternali \& Tomassetti, 2012). Aumer \& Binney (2009) constructed a model to fit the data that also favored a steady increase of velocity dispersion with time.

In fact, the in-plane components of the GCS stars do not have simple Gaussian velocity distributions (Fig. 5), as first deduced from the Hipparcos data by Dehnen (1998). Not only is the overall distribution of the $V$ components quite skew with an "asymmetric drift" $(\bar{V}<0$ as expected, BT08; Schönrich \& Binney, 2012), but the distribution is characterized by multiple "streams" that are distinct at a high level of significance (Bovy et al., 2009). The streams are both far too massive and have a spread in metallicities to be dissolved star clusters (Famaey et al., 2007); (Bensby et al., 2007); (Pompéia et al., 2011). Hahn et al. (2011) examined the nearby stars of the Sloan Digital Sky Survey (SDSS York et al. , 2000) and RAdial Velocity Experiment (RAVE Steinmetz et al., 2006) finding similar, but less distinct, substructure. Blurring of the velocity structures is to be expected for stars in these larger surveys, which do not have Hipparcos-quality astrometry and distances, with consequent loss of precision in the sky-plane velocity components. Antoja et al. (2012) traced some of these features in somewhat more distant stars of the RAVE survey.

The substructure in Fig. 5 probably arises from the dynamical influence of density perturbations in the disk and a number of attempts have been made to model it. De Simone et al. (2004) found that multiple, imposed transient spiral perturbations were able to create qualitatively similar substructure in the stellar velocity distribution. On the other hand, individual features have been interpreted as responses to assumed models for the bar (Dehnen, 2000; Minchev et al., 2010, see also Kalnajs 1991), or to specific spiral models (Quillen \& Minchev, 2005); (Pompéia et al., 2011); (Antoja et al., 2011), or both (Quillen, 2003); (Chakrabartv, 2007); (Antoja et al., 2009). Finally, Sellwood (2010, see also Hahn et al. 2011 and McMillan $2011,2013)$ did not need to adopt a perturbing potential, but instead used action-angle variable analysis to identify the Hyades stream as resulting from scattering by a recent Lindblad resonance. It is likely that the different streams have different origins and a combination of these ideas would be needed to explain all the features.

Returning to Fig. 4 the data show that the second moments of the peculiar velocities differ in all three components - i.e., the velocity ellipsoid of nearby disk stars has a triaxial shape that apparently grows roughly homologously with age. Seabroke \& Gilmore (2007) argue that the multiple streams in the $U-V$ plane (Fig. 5) make the second moment a poor measure of the velocity spread. However, the distribution in the $U-W$ plane is much smoother and it is worth noting that the rising trend of the $V$ component in Fig. 4 maintains a constant fraction of the radial component at the ratio expected from epicycle theory (BT08, Eq. 8.117) in a galaxy with an approximately flat rotation curve.

In Galactic components, the dispersion in the radial direction is the largest, the azimuthal component is intermediate, while the smallest is the component normal to the disk plane, being only about half as large as the radial velocity dispersion. A flattened shape appears to be representative of that in other 
disk galaxies (Bottema, 1993); (Gerssen et al., 2000); (Herrmann \& Ciardullo, 2009); (Bershady et al., 2011), although Gerssen \& Shapiro (2012) claim evidence that the axis ratio varies along the Hubble sequence.

Smith et al. (2012) present a study of local disk kinematics using the "Stripe 82" data from SDSS, although they make no attempt to assign ages to stars. Instead they divide stars by metallicity and present-day $z$-height below the disk plane and devise a procedure to estimate separate dispersions of the thin- and thick-disk stars that, however, must become increasingly difficult for metal-poor stars and those at large distances from the midplane. While their results for thin-disk stars with $-0.5<[\mathrm{Fe} / \mathrm{H}]<0.2$ are consistent with those from other studies, they found the velocity ellipsoid is distinctly rounder for more metal-poor stars that also have higher velocity dispersions.

Lewis \& Freeman (1989) found that the velocity dispersion in the Milky Way disk has a steep outward gradient over a wide radial range, as the above-cited studies also found in other galaxies. A gradient is, of course, expected on local stability grounds (Eq. 18), but the radial gradient must somehow combine with the velocity ellipsoid shape and disk surface density to create a vertical thickness scale that appears to be independent of radius for many galaxies (van der Kruit \& Searle, 1981); (Kregel et al., 2002). This conspiracy of disk properties has yet to be fully explained.

\section{Scattering by spirals}

Lynden-Bell \& Kalnais (1972) showed that, away from resonances (§II.H), a spiral perturbation that grows and decays adiabatically (on a time scale long compared with orbital and epicycle periods) leaves the stellar motions unchanged. Stars do work on, or receive energy from, a potential perturbation as it grows, but these changes are undone as the wave decays, leaving only oscillatory ripples in the phase-space density that average to no change (Carlberg \& Sellwood, 1985), except at resonances.

Wave-particle interactions at resonances do, however, cause lasting changes to the orbits of stars, and Lynden-Bell \& Kalnajs (1972) showed that stars at the ILR lose angular momentum on average, while those at the OLR gain. Changes at corotation could be of either sign, depending on the sign of the gradient of the angular momentum density of stars around the resonance (see (II.F).

The changes given by Eq. (20) become $\delta$-functions at resonances in the limit $\Im(\omega)=\gamma \rightarrow 0$, which Lynden-Bell \& Kalnais (1972) take. ${ }^{4}$ However, changes are smooth when broadened by time dependence, and

\footnotetext{
${ }^{4}$ Note the important aspect of trapping at resonances by a steady or slowly growing disturbance is not captured by Eq. (20).
}

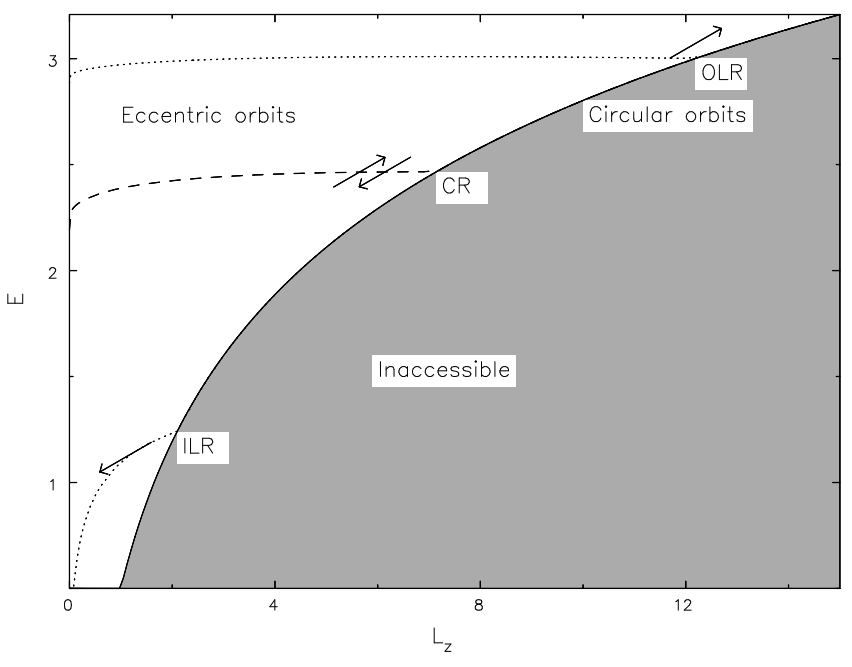

FIG. 6 The Lindblad diagram for a razor-thin disk galaxy model. Circular orbits lie along the full-drawn curve and eccentric orbits fill the region above it. Angular momentum and energy exchanges between a steadily rotating disturbance and particles move them along lines of slope $\Omega_{p}$ as shown. The dotted and dashed lines are the loci of resonances for an $m=2$ perturbation of arbitrary pattern speed.

Carlberg \& Sellwood (1985) compute the lasting changes under the assumptions that the wave both grows and then decays exponentially.

In a rotating, but otherwise steady, nonaxisymmetric potential test particles conserve neither their energy, nor their angular momentum, but Jacobi's integral,

$$
I_{J} \equiv E-\Omega_{p} L_{z},
$$

is conserved. Thus changes in energy and angular momentum are related as $\Delta E=\Omega_{p} \Delta L_{z}$. This is illustrated in Fig. 6] which is drawn for the midplane of an axisymmetric potential (see also Lynden-Bell \& Kalnajs, 1972, their Fig. 2). Circular orbits have the minimum energy $E_{c}$ for a given $L_{z}$, which marks the boundary of the shaded region, while orbits with $E>E_{c}$ are eccentric. Equation (25) constrains particles that are scattered by a nonaxisymmetric perturbation to move along lines of fixed slope $\Omega_{p}$ as illustrated by the arrows, which are marked at the principal resonances because those are the only places where lasting changes occur.

There is an important difference between scattering at Lindblad resonances and at corotation. A star near corotation may suffer quite a large change in its angular momentum, but because $d E_{c} / d L_{z}=\Omega_{p}$ at this radius (Fig. 6), it neither gains nor loses random energy (to first order); all the energy change is invested in changing the radius of the guiding center (Eq. 6). This is a characteristic feature of radial migration (\$III.C). The situation is different away from corotation, where there is an excess of energy available to increase random motion, provided that stars inside corotation lose $L_{z}$ while those outside gain. 
Sellwood \& Binney (2002, their Eq. 6) showed that the first order change in radial action of a star caused by a single spiral wave is related to its change in angular momentum via

$$
\Delta J_{R}=-\frac{l}{m} \Delta L_{z} .
$$

Since $l=0$ at corotation, any $\Delta L_{z}$ causes no change to $J_{R}$, as noted. Furthermore, $\Delta L_{z}<0$ at the ILR where $l=-1$ and $\Delta L_{z}>0$ at the OLR where $l=+1$. Thus outward transfer of angular momentum from the inner to the outer Lindblad resonance causes $\Delta J_{R}>0$, or heating, at both, as is also clear from Fig. 6, A succession of transient spiral modes with a variety of pattern speeds will cause the in-plane components of stellar random motion to increase generally over the disk.

\section{Vertical heating}

Note that the changes caused by transient spiral modes increase only the in-plane random motions, not the component normal to the plane as predicted by Carlberg (1987) and confirmed by Sellwood (2013b). The reason (\$II.H) is that coupling between the spiral perturbation and vertical motion is expected to be very weak because the frequency $\nu$ of small vertical oscillations of a star near the midplane (Eq. 8) is generally high compared with the Doppler shifted frequency $m\left|\Omega_{\phi}-\Omega_{p}\right|$ at which it encounters the perturbation, making its vertical motion adiabatically invariant. While a majority of stars rise out of the harmonic region, the fraction that have a low enough vertical frequency to experience a vertical resonance with a spiral perturbation is believed to be quite small.

The discussion in the previous paragraph assumed Newtonian gravitational forces, and softening in simulations (§II.L) can change the behavior. Increasing the gravitational softening length weakens the restoring forces to the midplane, decreasing the vertical frequency and possibly allowing vertical resonances to become dynamically important (see Solway et al., 2012, their Fig. 9). On the other hand, simulations with small softening, but modest numbers of particles, may thicken due to relaxation (Sellwood, 2013b). Thus the modeling of disk thickening in simulations is somewhat delicate.

Coherent bending waves are another possible mechanism to increase the vertical velocity dispersion. The mechanics of bending waves is complicated (see Sellwood, 2013a, for a recent review). However, we do know that a bending wave may travel across a stable disk (Toomre, 1983); (Weinberg, 1991) until it is damped as it approaches a vertical resonance (Sellwood et al. , 1998), with the wave energy going into localized vertical heating. It is also known (Toomre, 1966); (Araki, 1985) that a disk in which the velocity ellipsoid is flattened such that $\sigma_{R} \gtrsim 3 \sigma_{z}$ will buckle and thicken until the axis ratio is approximately this value (Sellwood,
1996); (Rodionov \& Sotnikova, 2013). ${ }^{5}$ However, the velocity ellipsoid of local stars in the Milky Way is not flattened enough to be near this stability boundary.

Another possible heating mechanism is infall of cosmic substructure (e.g. Kazantzidis et al., 2009). While infall of massive clumps in their simulations, and those of others, is quite disruptive and can probably be excluded ( (IV.B) , a gentler bombardment by smaller clumps may cause more gradual heating. However, a prediction of Kazantzidis et al. (2009) is that satellite bombardment should create velocity dispersions that are roughly constant with radius, whereas data on the Milky Way (e.g. Lewis \& Freeman, 1989) indicate a strong decline with radius to distances well beyond the solar circle. Thus, while some heating by infall cannot be excluded, it is clearly not the dominant process.

Since the data (Fig. 4) show that the out-of-plane motions rise with about the same slope as the in-plane part, it seems unlikely that spiral waves, neutral bending waves, or buckling instabilities are important in setting the shape of the local velocity ellipsoid in the local Milky Way. It therefore seems that scattering by collective waves cannot be the whole story.

\section{Scattering by dense mass clumps}

Spitzer \& Schwarzschild (1953) argued that massive clumps of gas were needed to account for the increase of peculiar stellar velocities with their ages, and therefore hypothesized the existence of giant molecular cloud complexes (GMCs) long before their existence was established. Their original calculation of scattering by dense mass clumps was extended to 3D by Lacev (1984). In his analysis, as in the earlier work by Spitzer \& Schwarzschild (1953), the star-clump interaction was computed in the impulse approximation, in which scattering is assumed to occur over a distance that is short compared with both the size of the star's epicycle and the scale on which the galactic gravitational potential changes.

Lacey (1984) found that co-orbiting mass clumps are quite efficient at redirecting peculiar motions out of the plane, but rather inefficient at increasing them. He also concluded that cloud scattering should cause the vertical dispersion $\sigma_{W}$ to be intermediate between the radial $\sigma_{U}$ and azimuthal $\sigma_{V}$ components. This result seemed physically plausible on energy equipartition grounds: scattering by massive clouds redirects the peculiar motions of stars through random angles, and therefore isotropizes the motions as far as the epicycle gyrations allow.

However, this again is inconsistent with the data (Fig. 4), where $\sigma_{W}$ is the smallest component. In order to account for the observed flattened shape, Carlberg

\footnotetext{
5 This behavior is also affected by gravity softening.
} 


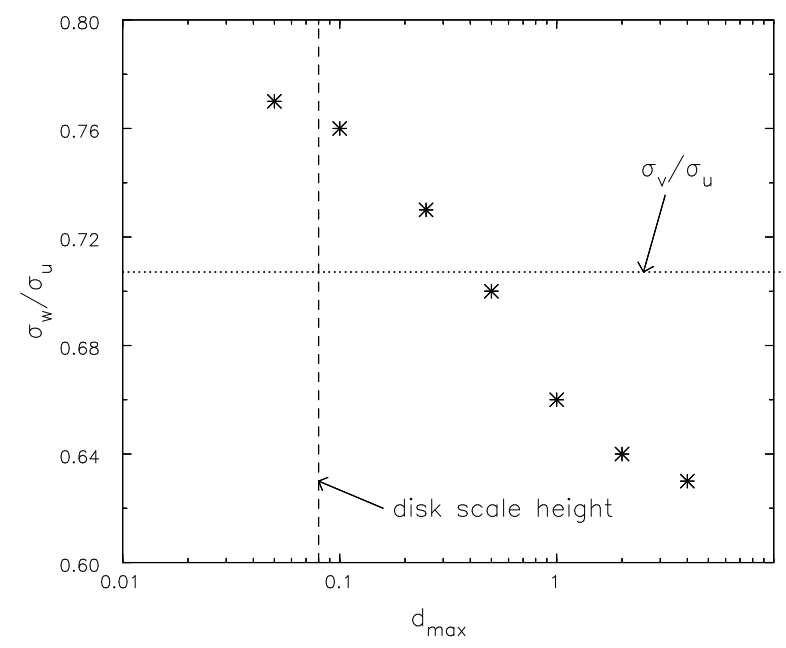

FIG. 7 The equilibrium axis ratio of the velocity ellipsoid of particles plotted as a function of the limiting range of the perturbation forces from the heavy particles. See (Sellwood, 2008b) for a description of the calculations.

(1987) and Jenkins \& Binney (1990) developed the plausible argument that the in-plane dispersion is driven-up more rapidly by spiral heating than scattering is able to redirect those motions into the vertical direction. Their argument seemed to offer strong support for the transient spiral mode picture (Sellwood, 2000), but it now appears to be incorrect.

Ida et al. (1993) found that cloud scattering alone would lead to the vertical component being the smallest, with the precise axis ratio of the velocity ellipsoid depending on the local slope of the rotation curve. Simulations by Shiidsuke \& Ida (1999), and others (see e.g. Villumsen, 1985); (Hänninen \& Flynn, 2002), confirmed their expectation.

The reason for the discrepancy with Lacey's prediction was clarified by Sellwood (2008b), who presented local simulations of scattering of test particles by massive co-orbiting particles. He artificially restricted the range of the gravitational forces from the heavy particles, which vanished at distances greater than some $d_{\max }$. Figure 7 shows the equilibrium ratio of the vertical to radial velocity dispersions $\sigma_{W} / \sigma_{U}$ plotted as a function of the adopted $d_{\max }$. The ratio settles to something close to the energy equipartition prediction when none but the closest heavy scatterers perturb the stars but, as the range of scattering was increased in separate experiments, the equilibrium ellipsoid gradually flattened and approached the shape predicted by Ida et al. (1993) for no cut off.

The flattened shape of the ellipsoid is determined by the fact that the perturbing clouds are located within the disk, leading to an aspherical distribution of impact parameters, with the consequence that deflections from the more distant clouds preferentially redirect the in-plane velocity components. Lacey (1984) and Binney \& Lacey (1988) neglected distant encounters, and therefore missed this effect. However, the familiar argument that every decade in distance makes an equal contribution to scattering also ceases to hold in disks (\$I.B), and the contribution to scattering by clouds that are more distant than several disk scale heights drops away rapidly. Thus it is the clouds at in-plane distances of just a few disk thicknesses that do most of the redirecting.

Smith et al. (2012) confirmed the predicted velocity ellipsoid shape for the metal rich stars in their data, but reported a rounder ellipsoid for the hotter, and probably older, metal-poor stars. Further work is needed to confirm this metallicity dependence, which may have been biased by the difficulty of separating thin- from thickdisk stars. Note that Holmberg et al. (2009) (see Fig. (4) found an ellipsoid shape that was almost constant with age, and at most only mildly rounder for the older stars.

\section{Collective effects}

The preceding calculations of scattering by mass clumps ignored all collective effects. Not only are disk galaxies subject to spiral perturbations, which themselves scatter stars, but the co-orbiting GMCs induce a collective response from the surrounding stellar disk (Julian \& Toomre, 1966) that substantially enhances their effective mass, a complication that is ignored in most studies of cloud scattering. An exception was provided by Toomre \& Kalnajs (1991), who studied scattering by both a density perturbation and the supporting response from the surrounding matter. The density fluctuations in their local simulations arose from the shot noise of the particles, while the same particles also took part in the supporting response. By applying a radial damping term, they may have unwittingly prevented the growth of instabilities (Sellwood, 2012), making their work a particularly clean calculation of the heating rate due only to the polarized disk response to co-orbiting mass clumps.

Since molecular gas is mostly (Nieten et al., 2006); Koda (2009); (Gratier et al., 2010); (Efremov, 2010), but not entirely (e.g. Corder et al., 2008); (Schinnerer et al. , 2013), concentrated in spiral arms it is probably futile to draw a sharp distinction between spiral arms and the wakes of dense gas clumps, and a correct treatment would be to calculate the effects of spiral formation and gas dynamics in the combined star and gas disk. Binney \& Lacey (1988) took a step in this direction, but a full calculation may remain unreachable for some time if one tries to include a self-consistent treatment of the formation and dispersal of the gas clumps: molecular gas concentrations probably grow in the converging gas flow into a spiral arm, and are subsequently partly dispersed by star formation. D'Onghia et al. (2013) also showed that massive clumps provoke spiral responses, but the spiral activity probably included some self-excited collective modes, since it persisted after the perturbers were removed.

Thus the studies of scattering in disks reviewed earlier 
make the simplifying assumption that spirals and mass clumps are distinct agents. This assumption at least separates the problem into tractable pieces. Perhaps it can be justified if the wakes of cloud complexes can be lumped with spirals into a single scattering agent that is distinct from the clouds that caused them.

\section{Conclusions on scattering}

We now understand that the velocity ellipsoid in the solar neighborhood is flattened as expected from scattering by GMCs (Ida et al., 1993). While the clouds efficiently redirect peculiar velocities to maintain the observed shape of the velocity ellipsoid, they are not thought to be responsible for much heating.

The magnitude of the peculiar velocities of the intermediate age stars exceeds what cloud scattering could achieve (Lacev, 1991). The old explanation for this, which may not be valid because it neglects the cumulative effect of intermediate distance encounters, was that the efficiency of scattering by clouds decreases as stars spend more time outside the cloud layer that is largely confined to the midplane. Simulations by Hänninen \& Flynn (2002), that did include distant encounters, confirmed that GMCs alone are unable to account for the random motions of the oldest stars.

Thus some other agent, generally assumed to be the spirals, is needed to boost the rms velocities of intermediate age disk stars to their observed values. Even though spirals do not heat the vertical motions, they drive up inplane random motions that are efficiently redirected by GMCs, and the velocity ellipsoid maintains an approximately constant shape as its size increases, accounting for the observed trends in Fig. 4. This picture does not exclude the possibility that the high peculiar motions of the very oldest disk stars, also known as the thick disk, have a different dynamical origin ( $\mathrm{IV}$ ).

\section{Heating in simulations}

Note that the behavior in $N$-body simulations needs to be interpreted with caution. Section III.B.6 accounted for the observed peculiar velocities in the solar neighborhood using the combined action of two distinct mechanisms: heating by spirals with the random motions being redirected by molecular clouds. Simulations support spiral patterns that may resemble those in galaxies and, if collisionless, should not thicken because they generally omit heavy particles to represent GMCs. ${ }^{6}$ Yet a few authors (e.g. Quinn et al., 1993); (McMillan \& Dehnen, 2007) have worried that disks thicken in isolated $N$-body simulations that are heated by spiral activity.

\footnotetext{
6 D'Onghia et al. (2013) included heavy particles, but did not discuss their effect on the velocity ellipsoid shape.
}

House et al. (2011) compared the vertical heating in a simulation with the solar neighborhood data. Their simulation, which was probably heated in part by spirals, included the cosmologically expected infall of pieces of substructure that could increase the vertical dispersion, and also modeled the full "gastrophysics" of cooling, star formation and feedback. However, they employed gas (and star) particles having the masses of GMCs, which therefore lacked the spatial and mass resolution to form dense clumps that are crucial to shaping the velocity ellipsoid.

Collisional relaxation, which is much more rapid in disks (Sellwood, 2013b, and 乌I.B), is a more likely explanation for redirecting in-plane motions to thicken disks in simulations. ${ }^{7}$ Thus, the simulated vertical heating rate, in particular, will depend on the number of particles employed, and comparison with the observed vertical heating of disk stars in the Milky Way (e.g. House et al., 2011) is premature without careful numerical convergence tests.

\section{Radial migration and mixing}

For years, the focus of spiral scattering was on heating at Lindblad resonances, and changes at corotation went unreported. Sellwood \& Binney (2002) were therefore surprised to find that a transient spiral mode causes greater angular momentum changes to stars at corotation than occur at the Lindblad resonances, as shown in Fig. 8. These more substantial changes had not attracted attention because they do not heat the disk (Eq. 26), and stars largely change places in a dynamically neutral manner. However, they do have important consequences for the distribution of chemical abundances among the disk stars (Roškar et al., 2008b); (Schönrich \& Binney, 2009a); (Minchev et al., 2013).

Changes to the guiding center radii caused by a series of transient spiral modes with corotation radii scattered over a wide swath of the disk will cause stars to execute a random walk in radius with a step size ranging up to $\sim 2 \mathrm{kpc}$. The resulting strong radial migration, called churning, has implications for abundance gradients and age-metallicity relations. The apparent metallicity gradient is also blurred by epicyclic motions, which can readily be subtracted for an individual star without having to integrate the orbit (e.g. Yu et al., 2012), since the guiding center radius of a star is determined only by its angular momentum (Eq. 6).

The topic of radial migration is bedeviled by the fact

\footnotetext{
7 McMillan \& Dehnen (2007) found that thickening was suppressed when the azimuth of every disk particle was randomized after every step, in order to suppress the growth of nonaxisymmetric disturbances. However, such a procedure must also largely inhibit two-body scattering, as well as all coherent responses from the surrounding disk.
} 


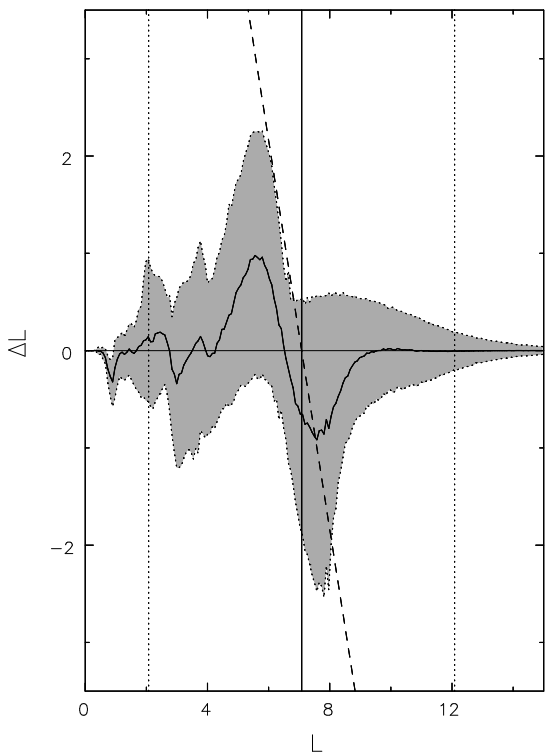

FIG. 8 Changes in angular momentum, $L$, resulting from a single transient spiral mode. The shaded region includes $90 \%$ of the particles and the solid curve shows the mean change. The vertical solid line marks the location of corotation, while the dotted lines mark the Lindblad resonances. The dashed line has slope -2. From Sellwood \& Binnev (2002).

that many authors have conflated the process first described by Sellwood \& Binney (2002) with the general redistribution of angular momentum that occurs with any nonaxisymmetric disturbance. The fact that spirals, bar formation, the excitation of responses by satellites, etc., redistribute angular momentum across the disk of a galaxy had been understood for decades. However, the recently discovered changes at corotation of a transient spiral mode have the two unique properties of neither heating the disk nor causing it to spread, as described next. All other forms of angular momentum transport do both these things. Even processes that are not associated with lasting angular momentum changes, such as increasing epicycle motions or of trapped particles that cross and recross a resonance, have been described as "radial mixing." While this phrase may be too deeply embedded to be redefined, "radial migration" and "churning" are less widely used and I suggest these terms be reserved to describe only the changes at corotation of a transient spiral mode.

While these other processes may play a role in redistributing matter radially, the low velocity dispersion of disks limits the extent to which heating at Lindblad resonances can have occurred, as discussed in III.B.2. Churning by spiral waves over the lifetime of the disk could, in principle, cause far more mixing with very limited heating, as found by Sellwood \& Binney (2002) and Solway et al. (2012) (see Fig. 9).

\section{Mechanism of radial migration}

Stars near corotation move slowly with respect to the spiral perturbation and therefore experience almost steady forcing from the wave, which allows large changes to build up - a process that is analogous both to surfing on ocean waves and to Landau damping in plasmas, although the consequences differ. Stars orbiting just behind the density excess are attracted forward by it and therefore gain angular momentum. However, the result of gaining angular momentum is that the star moves onto an orbit of greater guiding center radius (Eq. 6), and its angular frequency about the center therefore decreases. If the star were just inside corotation and therefore gaining on the density excess, the change can cause it to rise to a radius just outside corotation where it begins to fall behind. This behavior is described as a horseshoe orbit. Conversely, stars just ahead of the perturbation are pulled back, lose angular momentum and sink inwards, where they orbit at higher frequency. Those outside corotation, where the perturbation gains on them, could lose enough angular momentum to cross corotation and begin to run ahead of the wave. As long as the gradient $\partial f / \partial L_{z}$ is fairly shallow, roughly equal numbers of stars gain as lose, and they largely change places. The process affects stars with small peculiar velocities most strongly, since larger epicyclic motion leads to less coherent forcing by the spiral potential.

Were the spiral potential to maintain a fixed amplitude, stars on horseshoe orbits would be described as trapped. As they are moving slowly with respect to the wave, it would take them a long time to reach the next density maximum where the changes just described would be exactly undone. However, if the amplitude of a transient spiral mode has decreased by the time the star reaches the next density peak, it may no longer be trapped and will continue to move with a lasting change to its angular momentum.

Adopting variables suited to motion near corotation of a steady potential perturbation, Sellwood \& Binnev (2002) found that the radial extent of the region where these horseshoe changes occur varies as the square root of the perturbation amplitude, and therefore widens as a perturbation grows. At the same time, the more distant "trapped" stars move more rapidly in the frame of the perturbation, and the shortest period of a trapped star decreases as the inverse square root of the potential amplitude. They found the spiral was strong for less than half the horseshoe period for most trapped stars, which therefore undergo a single change.

Horseshoe orbits are also responsible for limiting the amplitude of the spiral. For a disturbance to grow, the response of the stars to the growing potential must reinforce the perturbed density, at least until it saturates. Sellwood \& Binney (2002) also argued that the maximum amplitude of a spiral is limited by the widening horseshoe region where stars are driven away from, instead of toward, the density maximum. This change kicks 
in suddenly because growth is linear in the disturbance potential, but the horseshoe region grows as its square root.

\section{Other radial mixing processes}

Any process that redistributes angular momentum mixes stars and gas that originated at different radii, and even the transient spiral modes that churn the disk also transport angular momentum to a much lesser degree. However, in contrast to the changes brought about by churning, redistribution of angular momentum across the disk always increases random motion and alters the large-scale surface density profile of the disk.

The role of bars as agents that mix the stars and gas of a disk has long been recognized (e.g. Hohl, 1971); (Friedli et al., 1994), and has received intense recent attention. The possible effect of resonance overlap between the bar pattern and the outer spiral was raised by Quillen (2003), see also Minchev \& Quillen (2006), and developed by Minchev \& Famaey (2010) for test particles in assumed nonaxisymmetric potentials of plausible bars and spirals. Since they adopted perturbations that grew to steady amplitudes, they clearly were not exploring the process described by Sellwood \& Binney (2002). Instead they found, as had Little \& Carlberg (1991, although without comment), that single perturbations created regions in which particles were simply trapped to cross and recross corotation with minimal angular momentum changes at the Lindblad resonances. However, simulations with two imposed disturbances revealed chaotic behavior when resonances of the two patterns overlapped, which Minchev \& Famaev (2010) described as "nonlinear" interaction. The substantially greater changes in the angular momenta of the particles were also associated with disk heating (Minchev \& Quillen, 2006). These studies raise the possibility that additional angular momentum transport could even occur were galaxies able to support multiple long-lived nonaxisymmetric structures. Quillen et al. (2009) also used the test-particle technique to show that the orbits of disk particles are "mixed" when perturbed by an orbiting satellite.

Results from $N$-body simulations are of greater interest, since the perturbations that cause the angular momentum changes are generated self-consistently and have physically reasonable time dependence. Brunetti et al. (2011) calculated diffusion coefficients in disks that form bars and spiral patterns finding, as seems reasonable, that angular momentum changes are lower in disks with higher $Q$ (Eq. 18). Minchev et al. (2011, 2012a) report simulations that formed bars and spirals, in which they claim evidence of enhanced angular momentum changes due to resonance overlap. They also highlight disk spreading, which is largely due to the angular momentum changes during bar formation (see $\sqrt{\mathrm{V} . \mathrm{B}}$ ), and also prolonged changes at the corotation resonance of a bar, which are likely caused by particles that are trapped to

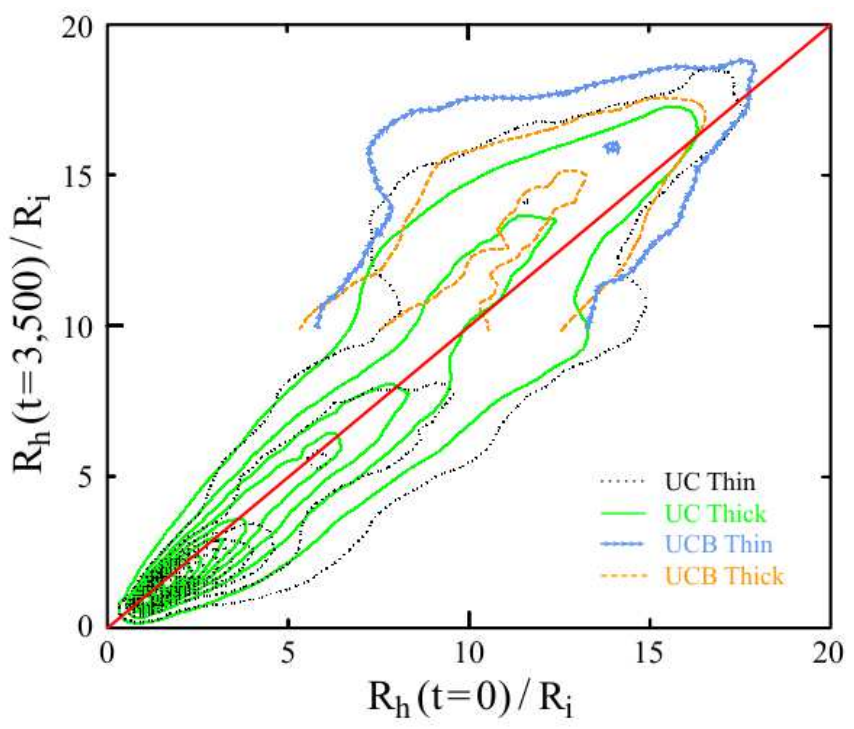

FIG. 9 Comparison of initial home radii with home radii after $\sim 10$ Gyr of evolution for particles in two simulations by Solway et al. (2012). For Milky Way scaling, the radial unit is $0.75 \mathrm{kpc}$. Simulation UC did not form a bar, whereas a bar did form in simulation UCB, and contours are drawn separately in different colors and line styles for the initially thin- and thick-disk populations. The barred region is omitted because $R_{g}$ (Eq. 6) cannot be defined in a strongly nonaxisymmetric potential.

cross and re-cross the resonance and therefore cannot sensibly be described as something as irreversible as mixing.

\section{Radial migration in simulations}

Roškar et al. (2008a) computed an isolated gas plus stars galaxy model that tracked star formation and metallicity evolution. The churning of the disk by a succession of transient spiral modes caused extensive migration. Some heating was caused by the smaller changes at the Lindblad resonances of spirals in their models, which is unavoidable, and they reported a change in the gradient of mean stellar age near the outer edge of the disk, which must have been created by the outward migration of particles. Roškar et al. (2008b) went on to demonstrate that migration led naturally to an age-metallicity relation similar to that in the Milky Way. A later work (Loebman et al., 2011) studied the formation of a thickened disk comprised of outward migrating stars with enhanced $[\alpha / \mathrm{Fe}]$ ratios and lower mean orbital speed. Bird et al. (2012) found that mixing is more extensive when spiral activity is invigorated by star formation, although the level of spiral activity in their models depended strongly on the "gastrophysical" prescription adopted. They showed that migration persists even for particles with large oscillations about the midplane, and they determined migration probabilities from their sim- 
ulations.

Figure 9 (from Solway et al., 2012) shows that the home radii of stars can migrate either inwards or outwards by many kpc, while the formation of a bar causes some comparatively mild additional mixing. Radial migration in the thick disk is only slightly weaker than in the thin disk, because the spiral potential that drives migration decays only slowly away from the plane. In fact, the slightly smaller average changes of the thickdisk particles were probably caused more by their larger in-plane random velocities than by their greater vertical oscillation (Solway et al., 2012, their Fig. 10). This is the likely reason that Bird et al. (2013) found that radial migration was less effective in the hotter, and generally older, particles of the inner disk of their model.

Roškar et al. (2012) presented a detailed study of radial migration in their simulations. They identified the locations, relative to the spiral density maxima, of particles that gained or lost large amounts of angular momentum and confirmed that even particles that moved rapidly over a large radial distance remained on nearcircular orbits.

\section{Adiabatic invariants}

One of the advantages of using action-angle variables to describe the motions of stars (乌I.D) is that the actions are adiabatic invariants. Broadly, this means that they are conserved quantities when the orbit of the star is subject to slow changes, except where resonances arise (see BT08, \$3.6 for a more careful statement). For example, Lynden-Bell (1963) used the invariance of radial action of a star to argue that orbital eccentricity would be invariant during gradual changes to the potential with no change to the angular momentum of the star. However, transient spirals change the angular momentum of stars at corotation without changing the radial action, and therefore eccentricity is not invariant during these changes.

Nevertherless, the radial action, $J_{R}$, is a useful adiabatic invariant during disk evolution except, of course, from the nonadiabatic changes at Lindblad resonances, where $l= \pm 1$ in Eq. (26). For nearly circular orbits $J_{R} \rightarrow \kappa a^{2} / 2$, with $a$ being the radial excursion of the star (Lynden-Bell \& Kalnais, 1972). For a well-mixed set of stars of fixed $J_{R}$, we have $\left\langle a^{2}\right\rangle=2\left\langle v_{R}^{2}\right\rangle / \kappa^{2}$ in the epicycle approximation, and therefore $J_{R} \approx\left\langle v_{R}^{2}\right\rangle / \kappa$. Thus, during radial migration $\left\langle v_{R}^{2}\right\rangle \propto \kappa$ for this group of stars, i.e. their radial dispersion decreases during outward migration, as found by Minchev et al. (2012b), and vice versa.

Solway et al. (2012) also showed that vertical action $J_{z}$, and not vertical energy, is the conserved quantity when stars migrate. Their conclusion stood out far more clearly when vertical action was calculated exactly (Eq. 9), than when the simple epicycle approximation was used (e.g. Minchev et al., 2012b). A somewhat puzzling finding by Solway et al. (2012) was that $J_{z}$ was con- served on average, but not precisely for individual particles; which may have been due to gradual relaxation that afflicts all $N$-body simulations of disks (Sellwood, 2013b).

Since its vertical action is adiabatically invariant during migration, the vertical oscillation amplitude of a star varies with the strength of the vertical restoring force, which in turn changes with disk surface density. A group of particles that have migrated outward in a nongrowing disk of declining surface density must have an increased scale height and a decreased velocity dispersion (Schönrich \& Binney, 2012); (Roškar et al., 2013). The vertical thickness would be squeezed by the increasing mass density that occurrs in a growing disk, but younger stars formed in the outer disk must still reside in a distinctly thinner layer than that of the outward migrating stars.

\section{Tests for radial migration in the Milky Way}

Currently, there has been no decisive test to confirm that radial migration really does occur in the Milky Way disk or elsewhere. But there are a number of strands of indirect evidence to suggest that the mechanism does occur.

Haywood (2008) found evidence for radial migration in a study of the metallicity distribution of solar neighborhood stars that suggested some stars were formed elsewhere in the disk. Lee et al. (2011) claim evidence for radial migration in the thin disk on the grounds that metallicity is uncorrelated with orbital eccentricity, but found a decreasing orbital velocity with metallicity in the thicker disk. Yu et al. (2012) reported a decreasing metallicity gradient with age of Milky Way thin-disk stars, which is the expected consequence of radial migration.

Bovy et al. (2012b) corrected data from SDSS for the selection function of the survey to determine the properties of the underlying stellar population. They found a continuous distribution of abundance-dependent disk structure with increasing scale height and decreasing scale length which they argued strongly favors "insideout" disk formation combined with gradual internal evolution through mechanisms such as radial migration. Both Bensby et al. (2011) and Cheng et al. (2012), in separate studies of quite different stellar populations, also found a short scale length for thick-disk stars, which are less well mixed because these populations are dynamically hot.

Haywood (2012) concluded that the metallicity distribution of disk stars seemed consistent with some degree of migration, but drew attention to a number of puzzling features. He cited very tentative evidence of a steplike feature in the radial distribution of metallicities (Hill et al., 2012) that, if confirmed, suggests that migration in the Milky Way may not have been efficient. However, Yong et al. (2012), in a study of open star clusters in the disk of the Milky Way, did not find a disconti- 

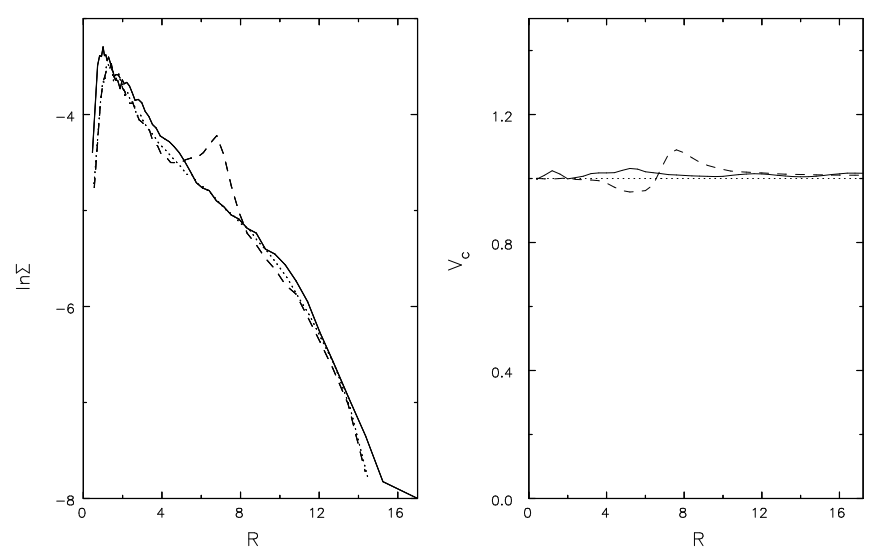

FIG. 10 The evolution of the surface density (left panel) and the total rotation curve (right panel) in the simulation described in $\amalg$ III.D The dotted lines show the initial unperturbed Mestel disk, the dashed lines are drawn after a ring of material is added to the disk centered on $R=7$, and the solid lines show the distribution five rotation periods later.

nuity in the abundance gradient at the solar radius, but instead found evidence for a change to a shallower slope at around $12 \mathrm{kpc}$.

Much stronger tests will emerge from current and future surveys. The Gaia mission (Perryman et al., 2001) will yield the kinematics of stars over a large fraction of the Galaxy in overwhelming detail. More detailed kinematics and chemical abundance measurements are being, or will soon be, collected in surveys such as APOGEE (Allende Prieto et al., 2008), LAMOST (Deng et al., 2012) and ARGOS (Freeman et al., 2013) see also Rix \& Bovy (2013). Indeed, one of the principal goals of the HERMES survey (Bland-Hawthorn et al., 2010) is to unravel the history of radial migration in the Milky Way.

\section{Smoothing rotation curves}

The rotation curve, or circular speed as a function of radius, is remarkably smooth for most galaxies (see Sofue \& Rubin, 2001, for a somewhat dated review). There is no feature even where the central attraction shifts from being baryon-dominated to dark matterdominated, which Bahcall \& Casertano (1985) described as a "disk-halo conspiracy." A few authors (e.g. Kalnajs, 1983); (Kent, 1986); (Palunas \& Williams, 2000) have drawn attention to "bumps and wiggles" in long-slit rotation curves, some of which correspond to photometric features in the light profile. While this is undeniable evidence for significant mass in the disk, the underlying cause of these small-scale features may be spiral arm streaming rather than substantial fluctuations in the radial mass profile of the disk.

Spiral instabilities may also be responsible for featureless rotation curves, as first argued by
Lovelace \& Hohlfeld (1978). While running simulations with a different purpose, Sellwood \& Moore (1999) noted that as the disk grew in their models with a dense central mass and a (rigid) cored outer halo, the mass distribution in the disk rearranged itself such that the resulting rotation curve was remarkably featureless. They noted that they obtained this result with a number of differing rules for the angular momenta of particles added to the disk.

A more controlled example is illustrated in Fig. 10 (Sellwood, in preparation). In this simulation, the initial model was the Mestel disk that has a circular speed independent of radius shown by the dotted line in the right panel and, in this case, only one-third of the central attraction is from the disk with remaining two-thirds due to a rigid halo. The dashed lines show the consequences to the surface mass profile and rotation curve of adding, over a period of less than one disk rotation, an extra ring of matter composed of live particles to this archetypal featureless model. The model quickly developed strong spiral patterns and after just five rotation periods, the rotation curve and the surface density distribution became featureless again, as shown by the solid lines.

The spirals that developed in this model were the result of two unstable modes that were provoked by the density ridge. Local stability analysis of a disk with a ridgelike density feature (Sellwood \& Kahn, 1991) predicts that, for each sectoral harmonic, the normal modes are wave pairs with corotation on opposite sides of the ridge. However, only those wavelike distortions to the ridge that can excite a strong supporting response from the surrounding disk are unstable. The angular periodicity that excites the strongest supporting response depends on the $X$ parameter of swing-amplification theory (Eq. 19), and the most rapidly growing pair of modes is for $m=3$ for the disk mass in this simulation. As the amplitudes of the modes rise, horseshoe orbits (\$II.C.1) develop at both corotation resonances but, unlike in a featureless disk, the presence of the ridge causes the resulting $L_{z}$ changes to be strongly out of balance at corotation for both modes. Thus far more particles are removed from the ridge than are added to it, causing the density profile of the disk to flatten, as shown by the solid curves in Fig. 10 .

Thus it seems that the distribution of angular momentum in the baryonic material that makes galaxy disks does not need to be able to account for the featureless character of most galaxy rotation curves, and small-scale variations in any reasonable distribution will be erased by spiral activity. The experiments of Sellwood \& Moore (1999), together with results from more realistic modern simulations (Abadi et al., 2003; Agertz et al., 2011) hint that this effect may be substantial enough to control the overall shape of the rotation curve, although further work is needed to establish this more interesting conclusion. 


\section{E. Angular momentum redistribution}

For a star in a rotationally-supported disk, the angular momentum $L_{z} \sim R V_{c}$ is much greater than the typical radial action $J_{R} \sim a \sigma_{R}$, with $a$ being the radial excursion. Thus, $J_{R} \ll L_{z}$, and Eq. (26) therefore requires that $\Delta L_{z} \ll L_{z}$ at Lindblad resonances. The implication is that spirals do not cause large changes to the distribution of angular momentum among the stars of a disk, as was borne out in $N$-body simulations (Sellwood \& James, 1979); (Bird et al., 2013). The largest changes to the distribution of $L_{z}$ in a disk occur during bar formation ( $\mathrm{g}$.B $)$, which has long been known (Hohl, 1971) to create a high level of random motion although, even after this event, $J_{R} \ll L_{z}$ in the outer disk.

This conclusion is not inconsistent with the large changes in $L_{z}$ at corotation, that cause stars to diffuse through the disk with little heating (\$II.C). Changes at corotation cause stars largely to exchange places, with only a very minor net change (if any) to the large-scale distribution of angular momentum within the disk. Even the smoothing of features in the rotation curve (\$II.D) causes only a localized smoothing of the angular momentum distribution.

Note that in growing galaxy disks, the specific angular momentum of infalling gas is expected to rise over time - the so-called "inside-out" growth of disks (e.g. Matteucci \& Francois, 1989); (Samland \& Gerhard, 2003); (Bird et al., 2013). The distribution of angular momentum among the stars formed from this material is expected to differ from that of the old disk. In this case, the total distribution of angular momentum within the disk changes for a quite different reason.

Thus the dynamically cool disks of stars in galaxies testify that their large-scale distribution of angular momentum cannot have been greatly altered from that at the time the stars formed. This constraint from random motion does not apply to redistribution within the gas component, however, since random motion in the gas is quickly dissipated.

\section{DISK THICKENING AND SURVIVAL}

The disk of the Milky Way contains both a thin layer of young stars and a thicker layer of old stars. For a long time they were described as separate components, with intermediate age stars being included as part of the "thin" disk Gilmore \& Reid, 1983); (Liu \& Chabover, 2000); (Munn et al., 2004); (Jurić et al., 2008); (Ivezić et al., 2008). However, Bovy et al. (2012a) suggested that there is no clear distinction between the two populations but rather a continuous variation in thickness, metallicity and radial scalelength, with the oldest, most metal-poor and hottest component having the shortest radial scale length. Others have challenged this conclusion Bensby (e.g. 2013), arguing that the thick disk is a distinct component.
Whichever way this discussion is settled, the thick and thin terminology remains useful to distinguish the two ends of the thickness range.

Burstein (1979), Mould (2005), and others found evidence for a thicker layer of older stars in other galaxies, which Yoachim \& Dalcanton (2006) suggested may be more massive, relative to the thin disk, in lower mass galaxies. Furthermore, Comerón et al. (2011) suggested that the thick components may be more massive than previously believed.

The thin and thick disks of the Milky Way can be distinguished not only by their scale heights and velocity dispersions, but the thick disk lags in its net rotational velocity (Chiba \& Beers, 2000), contains older stars with lower metallicities (Majewski, 1993), its stars have enhanced $[\alpha / \mathrm{Fe}]$ ratios (Bensby et al., 2005); (Reddy et al., 2006); (Fuhrmann, 2008); (Ruchti et al. 2011); (Schlesinger et al., 2012); (Liu \& van de Ven, 2012). As noted Bensby et al. (2011) and Cheng et al. (2012) suggest a shorter radial scale length for the $\alpha$-enhanced thick disk, although their estimates are still quite uncertain. These distinctions are not clear cut, and the assignment to a population may depend somewhat on whether a spatial, kinematic, or chemical abundance criterion is applied (Fuhrmann, 2008); (Schönrich \& Binney, 2009b); (Loebman et al., 2011).

\section{A. Formation of thickened disks}

Whether the thick disk is or is not a separate component has important implications for its formation. A distinct component suggests some event, such as a minor merger (see $₫$ IV.B $)$ in the past, stirred up the old disk and what is now described as the thin disk began to form subsequently through gas accretion and star formation, creating two chemically and dynamically distinct populations. However, evidence of such an event could well be obscured by one or more of a number of other mechanisms that may also contribute to the currently observed properties.

In addition to the minor merger hypothesis, Abadi et al. (2003) proposed that the debris accreted from disrupted satellite galaxies could form part of the thick disk, but chemical analysis of thick-disk stars (Ruchti et al., 2010, 2011) argued against this suggestion. Brook et al. (2005) and Bournaud et al. (2009) have suggested that stars formed in a thicker gas layer during galaxy assembly could have given rise to a thick disk. A fourth suggestion is that stars migrating outward from the inner Galaxy would have a thick distribution. Both the simulations of Loebman et al. (2011) and the semianalytic model for Galactic chemical evolution that includes radial migration by Schönrich \& Binney (2009a), showed that outward radial migration of old stars from the inner disk can create a thick population of old, metal-poor, stars with enhanced $[\alpha / \mathrm{Fe}]$ ratios. Schönrich \& Binney (2009a b) and Scannapieco et al. 
(2011) pointed out that it naturally gives rise to both a thin and a thick disk, under the assumption that thick-disk stars experience a similar radial churning. This assumption was validated by Solway et al. (2012).

Sales et al. (2009) proposed a test, based on orbit eccentricity, to distinguish these formation mechanisms, that has not proven decisive (Dierickx et al., 2010); (Casetti-Dinescu et al., 2011); (Wilson et al., 2011), although it does disfavor the accretion scenario. In this context, it should be noted that the peculiar velocity components, even of stars having highly eccentric orbits, could be redirected by GMC scattering (III.B.4), weakening the power of such tests. Furthermore, it is likely that more than one of these mechanisms has been at play as the disk of the Milky Way has built up.

Sridhar \& Touma (1996) suggested that the thick disk was formed by "levitation", in which radially eccentric in-plane orbits were converted to near-circular inclined orbits through resonant trapping as the potential of the Galaxy became more flattened during disk growth. However, the observed orbital eccentricities in the thin and thick disks today are the other way around.

\section{B. Survival of thin disks}

The hierarchical model of galaxy assembly (\$I.A) is challenged by the thinness of disk galaxies (Tóth \& Ostriker, 1992), which are stirred and thickened by the infall of satellite galaxies (Quinn et al. 1993); (Walker et al., 1996); (Velazquez \& White, 1999); (Berentzen et al., 2003); (Read et al., 2008); (Villalobos \& Helmi, 2008); (Kazantzidis et al., 2009). The severity of the challenge to the current $\Lambda \mathrm{CDM}$ paradigm involves many questions that are not easily answered. Wyse (2009) summarizes the evidence that the thick disk of the Milky Way, and perhaps that of other galaxies (Mould, 2005), contains essentially no stars younger than $\sim 10^{10} \mathrm{yr}$. If this critical piece of evidence holds up, it implies that no gravitational disturbance to the disk could have scattered stars into the thicker layer throughout that time.

The survival of the so-called superthin galaxies (see e.g. Matthews, 2000) presents a similar challenge. They are believed to be low-surface-brightness galaxies viewed edge-on that are probably embedded in a massive halo. If the low-luminosity density represents a low disk mass density, then their disks are less coherently held together by their self-gravity than are normal disks, making them all the more fragile to gravitational perturbations. Thus, not only are these disks remarkably thin, but they would be more easily thickened by perturbations than would heavier disks.

The expected rate of infall of subhalos as a function of their mass can be estimated from simulations of the growth of dark matter halos in the appropriate cosmology (e.g. Purcell et al., 2009). However the infalling pieces of substructure can be tidally disrupted, and may merge into the smooth inner halo (Gao et al., 2011). The Sagittarius stream (e.g. Belokurov et al., 2006) provides a clear example of the tidal stripping of a satellite as it falls into the Milky Way halo.

If the core of a dwarf galaxy is dense enough to survive until it interacts strongly with the disk, it may deposit some of its orbital energy into the disk, the remainder being absorbed by the halo through dynamical friction (बVI) . A proper calculation of this process needs to take into account the damping of the vertical oscillation by dynamical friction (Quinn \& Goodman, 1986), the reorientation of the disk plane in response to the absorption of misaligned angular momentum (Huang \& Carlberg, 1997), and the excitation of bending waves that can travel some distance across the disk before depositing their energy into vertical random motion (Sellwood et al., 1998). The coherence of the disk needed to support these last two mechanisms depends both on its self-gravity and on the degree of random motion (Debattista \& Sellwood, 1999).

The simulations by Kazantzidis et al. (2009) reveal that the disk is significantly distorted and thickened by the infall of a sequence of massive subclumps. The larger clumps which arrived first caused the most disruption, while the smaller fragments did less damage. All three velocity components of the disk particles rose substantially, while the disk also developed a pronounced flare.

Many (e.g. Moster, 2010); (Villalobos et al., 2010); (Puech et al., 2012) have pointed out that gas infall subsequent to a minor merger can form a new thin disk, and that the attraction of the additional mass in the disk squeezes the thickened layer of older stars. However, stars formed prior to the merger remain in a thickened layer (see e.g. Brook et al., 2004); (Robertson et al., 2006); (Governato et al., 2009). The Milky Way may have a continuum of disk populations of increasing thickness and age (Bovy et al., 2012a); but if the conclusion of Wyse (2009) that the thickest subcomponent contains no stars with ages $\lesssim 10^{10}$ yr holds, then the disk could not have been gravitationally stirred for all that time. Such a constraint would present a significant challenge to current cosmological models.

\section{Challenge to radial migration models}

The old age of the thick disk also raises a challenge for radial migration models (Schönrich \& Binney, 2009b). If some thick-disk stars have migrated from the inner Milky Way through the action of spirals, why are they all so old? One might expect at least a tail of young stars that have migrated rapidly from the center, although there were very few in the simulation by Loebman et al. (2011). Solway and Sellwood (in preparation) suggest that the formation of the bar in the Milky Way prevented any stars born in the inner Galaxy from being caught up by the corotation resonance of spirals and carried to the outer disk. If this suggestion is correct, then we may be 
able to date the formation of the bar in the Milky Way from the oldest stars that have inner disk metallicities. Other processes may have added stars to the thick disk, but there is at least hope that the abundance ratios of some elements might be unique signatures that the star originated in the inner Milky Way.

\section{BARS}

A majority of disk galaxies contain a bar of some kind. Bars are clearly visible in some $30 \%$ of galaxies, as judged from SDSS galaxy images by the Galaxy Zoo project (Masters et al., 2011). A larger bar fraction is seen in near-infrared images (e.g. Eskridge et al., 2000); (Menéndez-Delmestre et al., 2007), in part at least because bars can be obscured by star-forming regions in later type galaxies (e.g. Block \& Wainscoat, 1991). The bar fraction rises still further when weak oval distortions and short bars are included (e.g. Marinova \& Jogee, 2007); (Reese et al., 2007). Yet even in these studies, some $30 \%$ of disk galaxies in the local universe still lack any trace of a bar.

The incidence of bars over cosmic time has been investigated in a number of studies, which face difficulties of morphological classification from small images, even with Hubble Space Telescope resolution, and of band shifting of the light distribution with redshift. Cameron et al. (2010), who reviewed previous work, concluded that the bar fraction in more massive galaxies has been constant since $z \sim 0.6$, but has increased in lower mass galaxies by about a factor of 2 over the same time interval. Sheth et al. (2012) also found that bars are less common in disturbed galaxies at high redshift. These findings seem consistent with a general picture that the bar fraction appears to be set after galaxies form and settle (Kraliic et al., 2012), which happens earlier in more massive galaxies. Bars are therefore believed to be old, long-lived structures.

Bars are also believed to have a greater extent normal to the disk plane than does the disk that hosts them, giving them a "peanut" shape when viewed edge on. Since we cannot see the faceon view in such cases, the evidence to support this interpretation of box-peanut bulges is indirect (e.g. Bureau \& Athanassoula, 2005). The inner Milky Way manifests such a shape (e.g. Blitz \& Spergel, 1991); (Weiland et al., 1994); (Strutskie, 2006). In fact, its peanut shape is so pronounced that it is described as an "X shape" (McWilliam \& Zoccali, 2010); (Nataf et al., 2010); (Ness et al., 2012); (Wegg \& Gerhard, 2013).

\section{A. Stellar dynamics of bars}

Sellwood \& Wilkinson (1993) gave a thorough review of barred galaxies. Although somewhat dated, I refer the reader to that review for a detailed account, and give

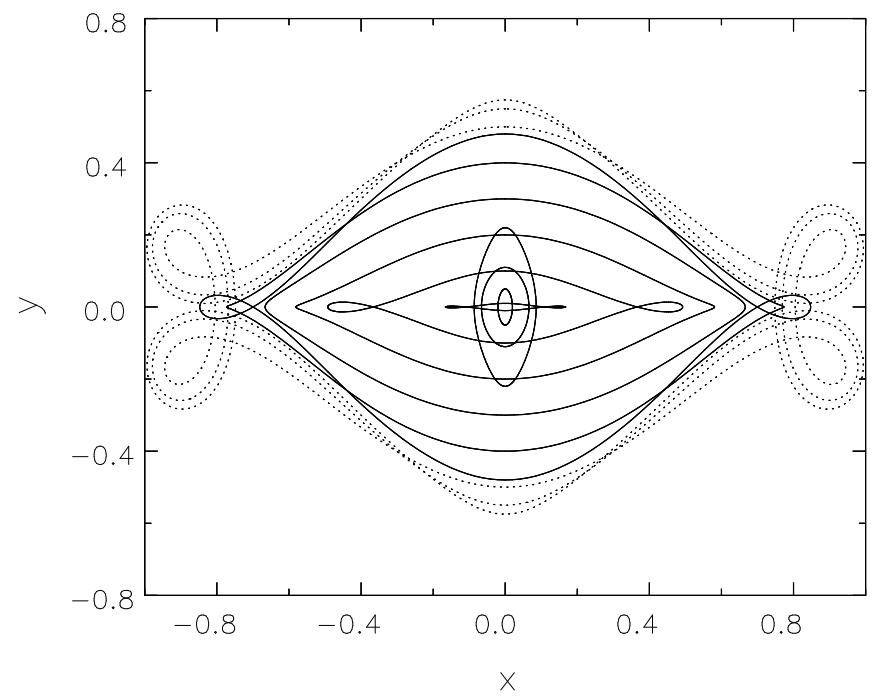

FIG. 11 The solid lines show examples, in a rotating bar potential, of important periodic orbits that close after two radial oscillations for every turn about the center: the 2:1 resonant families. Those orbits elongated parallel to the bar axis (horizontal) are members of the $x_{1}$ family. The $x_{2}$ orbits are elongated perpendicular to the bar. The dotted lines show three 4:1 resonant orbits (which close after four radial oscillations for every turn about the center) that may contribute to the somewhat boxy appearance of many bars.

only a brief outline of the basic dynamics of bars here.

A self-consistent stellar bar has a nonlinear dynamical structure that is most easily visualized in a frame that rotates with the bar. A large fraction of the stars in a bar move in the forward sense in the rotating frame on elongated orbits that are confined to the bar. Clearly, moving forward in the rotating frame implies, in an inertial frame, an orbit period about the center that is shorter than the figure rotation period.

Most of the orbits within the bar occupy regular parts of phase space, in which the stars are trapped about periodic orbits, and there are a number of resonant families of such orbits. Figure 11 shows the most important orbit families in the midplane of an idealized rotating bar, which has the simple effective potential ( $c f$. BT08 Eq. 3.103)

$$
\Phi_{\mathrm{eff}}(x, y)=\frac{1}{2} v_{0}^{2} \ln \left(1+\frac{x^{2}+y^{2} / q^{2}}{R_{c}^{2}}\right)-\frac{1}{2} \Omega_{p}^{2} R^{2},
$$

where $R^{2}=x^{2}+y^{2}, R_{c}$ is a core radius inside of which the potential is approximately harmonic, $q \leq 1$ is the flattening, and $v_{0}$ is the circular speed at large $R$ when $q=1$. As in BT08, the values are $v_{0}=1, q=0.8$, $R_{c}=0.03$, and $\Omega_{p}=1$.

Periodic orbits are described as resonant because they close in the bar-frame, and the solid curves show 2:1 families that close after two radial oscillations and one full turn about the center. The $x_{1}$ family is described as the "backbone" of the bar (Contopoulos, 1980), because the stars that are trapped around these orbits have a density 
distribution that is more elongated than the adopted bar potential. Since Poisson's equation requires the potential surfaces to be rounder than the density surfaces, the orbits that make up a self-consistent bar must be confined to a smaller region than is energetically accessible to them. This requires the majority of bar orbits to be regular, since chaotic orbits fill the volume bounded by their energy (or Jacobi constant, eq. 25).

Five Lagrange points occur in the bar potential, where a particle could remain stationary in the rotating frame. Four lie on the corotation circle, in an infinitesimal barlike potential: $\mathrm{L}_{1}$ and $\mathrm{L}_{2}$ lie on the bar major axis, $\mathrm{L}_{4}$ and $\mathrm{L}_{5}$ on the bar minor-axis, while $\mathrm{L}_{3}$ is at the bar center. In strong bars, $\mathrm{L}_{1}$ and $\mathrm{L}_{2}$ are closer to the center than are $\mathrm{L}_{4}$ and $\mathrm{L}_{5}$. The $\mathrm{L}_{1}$ and $\mathrm{L}_{2}$ Lagrange points in the potential used for Fig. 11] are very close to the points $(x, y)=( \pm 1,0)$,

Theorists generally agree that a self-consistent bar structure cannot extend farther from the center than the major-axis Lagrange points, because the stellar response outside corotation to forcing by a bar potential creates a density distribution that is elongated orthogonal to the bar direction. In addition, one cause of chaos in phase space is the overlap of resonances (Chirikov, 1979), and the increasing density of resonances (see ultraharmonic resonances in (II.H) as corotation is approached led Contopoulos (1980) to suggest that bars should end just before corotation. Elmegreen (1996) defined the useful dimensionless parameter

$$
\mathcal{R} \equiv R_{L} / a_{B}
$$

which is the ratio of the distance, $R_{L}$, of the Lagrange point $\mathrm{L}_{1}$ (or $\mathrm{L}_{2}$ ) from the galaxy center to the semi-major axis of the bar, $a_{B}$, and thus Contopoulos's argument is that $\mathcal{R}>1$ for all bars. While there is no compelling theoretical argument against $\mathcal{R} \gg 1$, which would be called a "slow bar", bar formation models, and observational evidence from barred disk galaxies (see $\$$ V.H) both indicate a strong preference for "fast bars" that have $\mathcal{R} \simeq 1.2$, although some exceptions have been claimed.

A second family of $2: 1$ orbits, known as the $x_{2}$ family, is also illustrated in Fig. [11. These orbits are found only deep inside the bar and are elongated perpendicular to it. They generally appear only in models with dense centers, where the orbital periods are short. The forced response of near-circular orbits can be calculated analytically for an infinitesimal bar perturbation (BT08 eq. 3.147), from which it can be seen that the driven orbit orientation differs by $90^{\circ}$ on opposite sides of all three major resonances (i.e. where $l=0, \pm 1$ in Eq. 15). Orbit integrations are needed in strong bars, where the orientation shift can be regarded as the generalization of the ILR to large amplitude perturbations. The existence of an ILR in the potential of the azimuthally averaged mass distribution is a necessary, but not sufficient, condition for the appearance of the $x_{2}$ family in a bar, since it can disappear as the bar strength is increased (Contopoulos \& Papayannopoulos, 1980). Even though the influence of the resonance can be recognized from the orbit structure, it is impossible to identify its location or radius in a strong bar because some $x_{1}$ orbits, which align with the bar, overlap spatially with the perpendicularly oriented $x_{2}$ orbits (Fig. 11).

The in-plane projections of many bars have distinctly "boxy" shapes (Kormendy, 1983); (Athanassoula et al., 1990); (Gadotti, 2011), suggesting that the parent orbits should have a somewhat rectangular shape. (The 3D shape is discussed later.) The dotted lines in Fig. 11 show three examples of 4:1 resonant orbits that are still elongated along the bar, whose existence may be related to the boxy shape of bars. Note that these orbits are found only close to corotation, where the orbit period in the rotating frame is long enough to allow four radial oscillations, and they therefore can be populated only in fast bars.

These 2:1, and perhaps also the 4:1, orbit families are the most important for bar dynamics, but many other less important orbit families have been found, even when motion is confined to a plane. The dynamics of motion in the third dimension is considerably richer (Pfenniger \& Friedli, 1991); (Patsis et al., 2002); (Skokos et al., 2002), with multiple commensurabilities possible between the vertical and in-plane frequencies. However, the most important orbits remain those that resemble the $x_{1}$ family when seen in projection. Those that oscillate about the midplane in either an archlike structure or a figure of 8 when viewed from the side are $2: 2: 1$ resonant orbits that complete two radial and two vertical oscillations for every turn about the center.

Note that the bar pattern speed is equal to the precession rate of the orbits that support it, and conversely all orbits precess at the bar pattern speed. In the absence of the bar potential, every orbit can still be regarded as a precessing ellipse (Kalnajs, 1973), but the precession rates of the orbits would no longer be equal with the larger orbits generally precessing at lower rates than the smaller. Thus the effect of the bar potential is to impose a common precession rate on orbits that would otherwise prefer to precess at a range of different rates. Clearly, the stronger the bar potential, the greater its ability to trap orbits to precess with it. Because the bar is a selfconsistent structure, its pattern speed can be regarded as an average, or "compromise," between the unforced precession rates of the orbits from which it is built. Thus bar pattern speeds must rise if mass accumulates in the bar center, since an increased mean density raises all orbital frequencies.

\section{B. The origin of bars}

\section{Global bar-forming mode}

It has long been known from both numerical simulations (Hohl, 1971); (Ostriker \& Peebles, 1973) and global stability studies (Kalnajs, 1972, 1978); (Jalali, 2007), that simple models of rotationally supported stellar disks 
are globally unstable. In the linear regime, the instability takes the form of an open two-arm spiral but, as it saturates, the shape straightens into a bar in the inner disk, while the spiral in the outer disk winds up and disperses. Hohl (1971) showed that the instability causes considerable rearrangement of the angular momentum in the disk ${ }^{8}$ and the azimuthally-averaged mass profile is substantially changed, with the inner disk becoming denser, while material is also spread far outwards.

The vigorous linear instability that creates the nonlinear bar is the result of a cavity-type gobal mode, or standing wave, in a massive disk. The linear instability can arise only if the combined mass distribution of the dominant disk, plus contributions from any bulge and dark matter halo, yields a quasiharmonic potential over the inner part, so that the rotation curve rises roughly linearly from the center before flattening around a radius $R_{\text {core }}$. In most circumstances, the group velocity (Toomre, 1969) of trailing spiral waves is directed away from the corotation radius, while leading waves propagate toward it, as illustrated for a centrally cusped potential in Fig. 2. In that figure, the trailing disturbance was absorbed at an ILR, but an inwardly propagating trailing spiral can travel all the way to the galaxy center if it does not encounter this resonance, and an $m=2$ disturbance easily avoids an ILR when the potential near the center is quasiharmonic. In that case, the incoming trailing wave reflects off the center into an outgoing leading spiral. The feedback loop is closed at corotation where the outgoing leading wave superreflects into an amplified trailing wave. At the same time, an outwardly propagating trailing wave is excited outside corotation that satisfies wave action conservation requirements. The mode is unstable because the wave train is amplified at corotation, and the instability typically exponentiates on the time scale of an orbital period.

The bar that results from this instability generally extends to a radius that is perhaps $20 \%-30 \%$ greater than $R_{\text {core }}$. The initial bar has a pattern speed that is often slightly lower than that of the eigenmode that caused it, and it almost fills its corotation circle. Thus bars are shorter in models with smaller harmonic cores (Sellwood, 1981), although something else can happen (see $\longdiv { \mathrm { V.B.3 } }$ ) if the core is very small or absent.

\section{Stabilizing mechanisms}

Because it grows through swing-amplification, this mode is highly unstable in massive disks with low velocity dispersion, whenever the feedback loop is open. If

\footnotetext{
8 Although the instantaneous angular momentum of an individual particle in a strongly nonaxisymmetric system changes continuously, particles in a settled bar stream round the bar in a steady fashion such that the distribution of their instantaneous values does not evolve.
}

the disk is massive enough to contribute most of the central attraction, then $1 \lesssim X \lesssim 3$ for $m=2$ disturbances (Eq. 19), and strong amplification occurs unless $Q \gtrsim 2$.

In mass distributions with quasiharmonic cores, i.e. those that allow ingoing waves to reflect off the center, the instability can be quelled either by a high degree of random motion (Athanassoula \& Sellwood, 1986) or by making the disk unresponsive to $m=2$ disturbances, by making $X \gtrsim 3$ (Eq. 19). This latter solution is that favored by Ostriker \& Peebles (1973), Efstathiou et al. (1982), and by Christodoulou et al. (1995) who correctly argue that bar stability can be achieved if a large fraction of the central attraction over most of the inner disk comes from unresponsive spherically distributed matter (bulge and halo). However, this is not the only, or even the most realistic, way to stabilize a dynamically cool disk.

After having elucidated the mode mechanism, Toomre (1981) predicted that the formation of a bar could be prevented if the in-going $m=2$ wave were unable to reflect off the center of the disk. The easiest way to prevent feedback through the center is to ensure that the wave encounters an ILR, where it will be absorbed as illustrated in Fig. 2,

Simulations of models with centers dense enough to force an ILR for most patterns (Sellwood, 1985); (Sellwood \& Moore, 1999); (Sellwood \& Evans, 2001) confirm that Toomre's proposed mechanism can indeed stabilize a disk in high-quality numerical work. These globally stable galaxy models have massive disks with realistic rotation curves and a moderate degree of random motion.

\section{Bar formation through nonlinear trapping}

However, Efstathiou et al. (1982) reported that bars formed in their simulations with equal vigor no matter how dense they made the central bulge, in apparent contradiction with Toomre's prediction. Sellwood (1989b) confirmed that bars formed in his similar simulations with dense bulges when the initial arrangement of the particles was random. But he also found that the same models did not form bars when the particles were uniformly spaced in azimuth around rings to reduce the initial level of shot noise in the low-order sectoral harmonics - a quiet start. This different behavior arose because the absorption of in-going spiral waves at an ILR is predicted to occur at small amplitudes only. The higher level of shot noise from randomly placed particles seeds larger amplitude disturbances that can overwhelm the ability of the ILR to absorb them. The resulting non-linear trapping of particles causes a bar to form that is superficially similar to that formed through the global linear instability. Had Efstathiou et al. (1982) employed a much larger number of particles, a less subtle way to beat down the level of shot noise, they should have found that dense bulges can indeed stabilize a disk, as Toomre predicted. Thus the bulge and halo masses required by the popular 
stability criteria proposed by Ostriker \& Peebles (1973), Efstathiou et al. (1982) and Christodoulou et al. (1995) simply do not apply to galaxies with dense centers, or to high-quality simulations of models with this property.

Shot noise from the $\sim 10^{10}$ disk stars in real galaxies must have a low amplitude. Larger density fluctuations caused by star clusters and GMCs still seem unlikely to trigger nonlinear bar formation. However, this nonlinear method of making bars in globally stable disks could still occur, for example, by a large amplitude perturbation caused by a tidal encounter or minor merger (see $\mathrm{g} . \mathrm{B} 5$ ).

\section{Slow trapping of orbits}

Lynden-Bell (1979), in an elegant piece of dynamical analysis, proposed a mechanism for the secular growth of bars in galaxies. He showed that eccentric stellar orbits could, under certain reasonable conditions, gradually become trapped into a rather slowly rotating bar structure. Orbits tend to align in the inner parts of galaxies, thereby reinforcing the bar, when the overall density distribution is not too sharply peaked toward the center. Lynden-Bell (1979) envisaged that the angular momentum loss from the inner part of the galaxy would be mediated by spiral patterns, as may have happened in the simulations of James \& Sellwood (1978). This mechanism can be important for the secular growth of bars discussed below (乌.C).

Even though the mechanism was originally envisaged as a slow trapping process, Polyachenko (2004, 2013) argued it may also form slow bars on a dynamical timescale. He argued that this was the cause of the weak, slow bars that formed in the simulations described in the appendix of Athanassoula \& Sellwood (1986), which had velocity distributions that were strongly radially biased. The mechanism has also been identified (Palmer et al., 1990) as the root cause of the radial orbit instability in spheroidal stellar systems with radially biased DFs.

\section{Bar formation through tidal encounters}

A number of studies of tidal interactions of satellite galaxies with disks have shown that bars are often triggered by the encounter (Byrd et al., 1986); (Noguchi, 1987); (Gerin et al., 1990); (Salo, 1991); (Maver \& Wadslev, 2004); (Romano-Díaz et al., 2008b). If the unperturbed disk were stable, bar formation could still occur through the nonlinear trapping mechanism described in $\$$ D.B.3.

Miwa \& Noguchi (1998) suggest that tidally induced bars might be slower, in the sense that $\mathcal{R} \gg 1$, than those formed through the usual bar instability. Curir et al. (2006) also report that bars seemed to form more readily in cosmologically formed halos with moving substructures than in cases where the halo is smooth and nonevolving. Berentzen et al. (2007) reported that inter- acting galaxy models with large gas fractions appeared to be less susceptible to bar formation than their gasfree counterparts, although the high numerical viscosity of the SPH method ( $\mathrm{V.E}$ ) may have had an undue influence on this conclusion. The variety of possible galaxy mass ratios, orbits, spin directions, gas fractions, etc., implies that the comparatively few simulations so far reported have barely scratched the surface of this vast multidimensional, parameter space.

Despite a number of studies to attempt to determine whether galaxies with nearby companions or those in dense environments are more likely to be barred, the results have generally been inconclusive. Skibba et al. (2012) review this work, and present a much larger study of their own, based on barred classifications from the Galaxy Zoo project, that appears to find a significant excess of bars in galaxies with moderately distant companions. Much more theoretical work and further observational studies (e.g. Méndez-Abreu et al., 2012) are required to determine the extent to which bars in real galaxies could be caused by interactions.

\section{Recurring bars?}

The changes to the distribution of both mass and angular momentum that result from this global instability are the largest that occur in an isolated disk (Hohl, 1971); (Debattista et al., 2006). However, for reasons given below, bar formation through a global instability is widely believed to happen just once in the life of most disk galaxies, and the associated large structural changes are not expected to recur.

Bournaud \& Combes (2002) and Combes (2008) offer a dissenting view, and found that the instability can recur in their simulations. The process of bar formation and dissolution ( $\mathrm{V} . \mathrm{F}$ creates much random motion, leaving the original stellar disk dynamically "hot" and unresponsive. However, gas settling onto circular orbits and forming new stars creates a new, dynamically responsive, component. Thus, they invoke a high gas accretion rate in order that the whole disk may again become bar unstable. Some of their simulations did form a second bar, in Bournaud \& Combes (2002) after the disk mass had roughly doubled, and Sellwood \& Moore (1999) presented an additional case.

However, it is hard to see how such behavior could recur repeatedly, since each cycle adds mass to the hot unresponsive disk population. Also, angular momentum changes associated with earlier bar formation will have made the disk more centrally concentrated, which is stabilizing as Sellwood \& Moore (1999) demonstrated. Furthermore, inside-out disk growth suggests that fresh gas is expected to be accreted less in the center, where it would most be needed, and more in the outer disk. 


\section{Continued growth of bars}

A globally unstable bar-forming mode generally has an open spiral form, which causes a large-scale rearrangement of the angular momentum in the disk. However, the spiral soon fades, leaving the bar as the only persistent feature and the outer disk dynamically much hotter. If the disk does not extend far beyond the bar, and the simulation does not include a live halo or any dissipative component, then very little further happens.

A more extended disk can support continuing spiral activity for a period, which generally has a lower pattern speed than that of the bar (Sellwood \& Sparke, 1988), and the duration of that activity can be extended, perhaps indefinitely, by mimicking dissipation. The apparent connection between the bar and the spiral pattern in such cases must be transitory, although Sellwood \& Sparke (1988) showed that contours of the nonaxisymmetric density distribution appear to join the bar to the spiral for a significant fraction of the beat period. Whether spirals in real barred galaxies are driven responses to the bar or distinct dynamical entities has proven harder to establish (Buta et al., 2009); (Meidt et al., 2009).

Although spiral patterns in simulations generally have different pattern speeds from that of the bar, the two nonaxisymmetric structures do interact. Generally, it is found that spiral activity is associated with bar growth, since spirals remove angular momentum from particles, allowing them to become trapped into the bar (Lvnden-Bell, 1979); (Sellwood, 1981); (Solway et al., 2012). This process causes the bar to slow as it grows, because it adds material to the bar that has a lower natural precession rate (see $\$ \mathrm{V.A}$ ), and the requirement $\mathcal{R}>1$ continues to hold. Note that spirals do not always cause bars to strengthen and can sometimes cause them to weaken, as described in $8 \mathrm{V.F}$

Tagger et al. (1987) and Masset \& Tagger (1997) argue that bars can drive spirals through nonlinear resonance coupling. In their picture, the location of corotation of the bar coincides with the ILR of the outer $m=2$ spiral, which has a lower pattern speed, and the coupling is mediated by a third mode, which may be axisymmetric $(m=0)$ or $m=4$. Similar ideas were proposed by Fuchs et al. (2005). There is no doubt that many spirals in simulations have pattern speeds of approximately the angular frequency for this to be a possible explanation, but the evidence for the third mode that would confirm it has proved more elusive.

Bars also grow in length due to dynamical friction with the halo $(\overline{\mathrm{VI}})$, and growth by this process can be extreme (Athanassoula \& Misiriotis, 2002); (Martinez-Valpuesta et al., 2006); (Villa-Vargas et al., 2009). Athanassoula \& Misiriotis (2002) show that a bar in a moderately dense halo continues to grow until it is as large as the disk from which it formed! Perhaps the mechanism proposed by Lynden-Bell (1979) operates in this context also, with secular bar growth caused by loss of angular momentum to the halo instead of to the outer disk. Of course, the bar slows as it grows in these cases also. Since we do not observe bars of this size, in relation to their disks (e.g. Erwin, 2005), it seems reasonable to conclude that halo friction, which is determined by the inner halo density (\$VI), is too mild for excessive bar growth to occur in nature.

\section{Buckling instability}

After a bar has formed and settled, it generally experiences a second instability that causes it to thicken out of the plane into a pronounced peanut shape, as first reported by Combes \& Sanders (1981). Combes et al. (1990) and Pfenniger \& Friedli (1991) suggested that thickening is caused by a vertical resonance, since gradual thickening also occurs in simulations in which the buckling mode is suppressed by forcing vertical symmetry of the potential about the midplane, but Friedli \& Pfenniger (1994) concede that thickening is more rapid when buckling is allowed. The asymmetric bending of the bar when viewed edge-on in many simulations (Raha et al., 1991); (O’Neill \& Dubinski, 2003); (Martinez-Valpuesta \& Shlosman, 2004); (Martinez-Valpuesta et al., 2006) is a clear indication that a dynamical buckling instability is the principal cause of the peanut-like shape. The buckling instability of a bar is believed to have formed the peanut shape of the Milky Way bulge (e.g. Shen et al., 2010); (Li \& Shen, 2012); Gerhard \& Martinez-Valpuesta, 2012), and additional kinematic data (Vásquez et al., 2013) seem to support this picture.

Buckling instabilities had been predicted for a stellar system with an excessively flattened velocity dispersion ellipsoid (Toomre, 1966); (Kulsrud et al., 1971); (Fridman \& Polyachenko, 1984). Araki (1985) showed that the instability is present in a uniform stellar sheet with a $\operatorname{sech}^{2}\left(z / z_{0}\right)$ vertical profile provided $\sigma_{z}<0.3 \sigma_{x}$, and this criterion appeared to be roughly correct in a global axisymmetric model (Sellwood, 1996). Simulations of the nonlinear evolution of the instability in strongly prolate systems (Merritt \& Hernquist, 1991), disks (Sellwood \& Merritt, 1994), and the rotating bars of interest here, reveal that the flattened system develops an increasing bend in the vertical direction until selfgravity is no longer able to confine the particles to the bending layer; the nonlinear evolution is a puffier system with less extreme velocity anisotropy. In the case of rotating bars, the disk in which the bar formed may have been quite stable to buckling when axisymmetric, but the formation of the bar creates an elliptical flow, with substantial streaming motion in the radial direction that has the same destabilizing effect on the bending dynamics as does random motion.

It is clear that the 2:2:1 resonant orbit family invoked by Pfenniger \& Friedli (1991) is the reason that the bar takes on a peanut shape. Orbits of this family domi- 
nate in rapidly rotating 3D bars (e.q. Pfenniger \& Friedli, 1991); (Martinez-Valpuesta et al., 2006); in the rotating frame, they close after two radial oscillations (as do the $x_{1}$ family in 2D) and two vertical oscillations, with the vertical excursions peaking when the particle is far from the center.

The buckling instability weakens the bar (Raha et al., 1991); (Martinez-Valpuesta \& Shlosman, 2004); (Debattista et al., 2004, 2006), and causes it to become slightly more centrally concentrated, as energy added to the vertical motions is removed from the horizontal. The peanut shape of the bar after the instability may also be affected by the degree concentration of the central mass: the central waist is more pronounced in models with a quasiuniform inner density distribution, while the thickness is more uniform when the central density is strongly peaked (Berentzen et al., 2007).

The peanut shape generally does not encompass the full extent of the bar, i.e. there is some flat bar outside the buckled inner part (Lütticke et al., 2000); (Athanassoula, 2005); (Gadotti et al., 2007); (Erwin \& Debattista, 2013), as has also been claimed for the Milky Way (Martinez-Valpuesta \& Gerhard, 2011), with the "long bar" seen in counts of the midIR sources (Benjamin et al., 2005) and variable stars (González-Fernández et al., 2012). Erwin \& Debattista (2013) also argue that not every bar thickens vertically and estimated that at least $13 \%$ of bars in galaxies have not buckled.

Unfortunately, the nature of the buckling instability in simulations depends on spatial resolution (or the gravity softening length) used in the $N$-body code. Codes, such as that used by Raha et al. (1991), which do not have many zones or softening lengths within the vertical thickness of the disk yield restoring forces to the midplane that are not as sharp as they should be. A soggy restoring force increases the spatial scale of the instability, leading to the simple low-order buckling mode reported by Raha et al. (1991). Subsequent models with better spatial resolution found that bars still thicken, but the buckling occurs on shorter length scales, causing less pronounced bends before the mode saturates.

The inclusion of a rigid mass component, especially a bulge or central mass concentration that is held fixed, also compromises the proper representation of the buckling instability. Such models provide an additional restoring force to the fixed center, whereas a fully mobile mass distribution should move in response to the bend in the thin component (Berentzen et al., 2007).

Martinez-Valpuesta et al. (2006) show that a bar that grows substantially in length may undergo a second buckling instability. Athanassoula \& Misiriotis (2002) also found that the extent of peanut appearance grew significantly as the size of bar continued to increase.

\section{E. Gas response to bar forcing}

As described in $₫$ II.K, the interstellar medium (ISM) in galaxies is not a simple fluid with a well-defined equation of state. Thus before running simulations to model the gas flow, one must first decide how best to approximate the dynamical behavior of the ISM.

One approach (e.g. van Albada \& Roberts, 1981); (Piner et al., 1995); (Kim et al., 2012) is to use a standard Eulerian hydrodynamic code with an isothermal equation of state, adopting a sound speed that is representative of the velocity spread of the clouds, typically between 5 and $10 \mathrm{~km} \mathrm{~s}^{-1}$ rather than the much lower thermal speed. These well-developed methods have the advantages of optional adaptive grid refinement (e.g. Kravtsov et al., 2002) and a low numerical viscosity, but they also attribute a pressure that resists compression in a converging flow where the physical properties of the gas suggest that we should expect strong dissipation through some kind of bulk viscosity.

Lagrangian methods have also been applied, the most popular of which is smooth particle hydrodynamics (hereafter SPH, see Springel, 2010a, for a review). The advantage of these methods is that they concentrate numerical resources in the interesting regions of high density, and self-gravity of the gas can readily be combined with the scheme used for the stellar particles. A known weakness of SPH is its inability to support some standard fluid instabilities, especially the Kelvin-Helmholtz instability (Agertz et al., 2007), but fix-ups have been developed (Read \& Hayfield, 2012); (Hopkins, 2013). Another weakness of all Lagrangian methods, such as "sticky" particle and "colliding" particle schemes as well as SPH, is the high numerical viscosity due to the finite radius (or kernel width) of the particles. This is of particular importance in spatially separated, but nearby counter streaming flows, such as can occur in strongly nonaxisymmetric potentials. Two nearby streams of oppositely flowing particles whose interpolation kernels overlap will clearly drag on each other, causing viscous dissipation that may be greatly over-estimated.

Springel (2010b) described a promising new hybrid Eulerian-Lagrangian method that adjusts the grid cell boundaries as the fluid flows. However, the number of published examples is so far rather small, and most are applied to galaxy formation, rather than to galaxy evolution.

Since no one method perfectly mimics the dynamics of the ISM, it is good to compare the behavior in any one problem using a variety of techniques. One has greater confidence in behavior that is reproducible by more than a single method.

\section{Flows in two dimensions}

Many have reported simulations of a massless gas component flowing in a rigidly-rotating bar poten- 
tial (a partial list of some the more important papers is: Sanders \& Huntley, 1976); (Sanders \& Tubbs, 1980); (Matsuda \& Isaka, 1980); (Schwarz, 1981); (van Albada \& Roberts, 1981); (Athanassoula, 1992); (Kim et al., 2012). These simulations used a variety of approximations to model the gas, but generally they found that gas within the bar region is driven inward toward the galaxy center, where it accumulates, while gas in the region outside corotation is driven outward.

Because the gas is moving highly supersonically, pressure is negligible and, except where shocks arise, the motion of a fluid element follows a ballistic orbit. Therefore, were shocks absent, mild dissipation would drive gas onto streamlines corresponding to periodic orbits in the potential. However, in most bar flows, the periodic orbits do not nest without intersecting others or themselves, as exemplified in Fig. 11, and shocks must form. Shocks form along the leading edges of the bar as it rotates, causing the gas to lose both energy and angular momentum. The loss of angular momentum occurs because the shocks skew the flow pattern with respect to the axis of the bar, and therefore the gas spends more than half its time on the leading side of the bar, where the nonaxisymmetric part of the bar potential applies a retarding acceleration. In all models except those that lack a central mass concentration, the inflow stalls at some distance from the center, which happens where the $x_{2}$ orbit family appears.

Observational evidence from barred galaxies (reviewed in Kormendy \& Kennicutt, 2004) suggests that something like the behavior just described also happens in nature. Prendergast (1962) appears to have been the first to associate shocks with the dust lanes that are generally seen on the leading edges of the bar, assuming the outer spiral to trail. Physically, a shock in simulations of the idealized ISM implies, in real galaxies, locations where streams of gas clouds undergo more frequent collisions, causing a change in momentum, and a large increase in density that gives rise to the dust lane. Steep velocity gradients across dust lanes can be detected in high resolution velocity maps (e.g. Weiner et al., 2001); (Hernandez et al., 2005); (Zánmar Sánchez et al., 2008) and massive gas concentrations are observed in the centers of barred galaxies (Sheth et al., 2005); (Regan et al., 2006); (Gerin et al., 1988); (Garcia-Barreto et al., 1991a). Large accumulations of gas, presumably having been driven inwards by the bar, are often found in circumnuclear rings (Garcia-Barreto et al., 1991b) (see §VII.A).

Extracting a reliable estimate of the inflow rate of gas from simulations is fraught with difficulties, however. The high numerical viscosity of some methods may enhance the inflow rate (Prendergast, 1983) but, even more insidious, is that the precise position of the shock, and therefore the magnitude of the gravity torque on the gas, is strongly affected by the choice of numerical scheme and parameters. Quillen et al. (1995) imaginatively took an observational approach to avoid these pitfalls, although other difficulties arise associated with accounting for all phases of the gas.

A small fraction of the radial flow may continue inward (Wada, 2004); (Kim et al., 2012), perhaps driven by weak spirals that are particularly prominent in dust (Carollo et al., 1998); (Martini et al., 2003). Again, the inflow rate in a simulation depends strongly on the numerical scheme and parameters (Kim et al., 2012) and Kim \& Stone (2012) found that including magnetohydrodynamics substantially increases the inflow rate in this region. However, it is clear from the observed build up of gas in the nuclear rings of real barred galaxies, that the inward mass flux interior to the ring must be lower than that which flows down the bar into the ring.

Wada \& Koda (2001) included self-gravity of the gas, as well as heating and cooling. But the more important limitation of most simulations mentioned in this section is the neglect of the bar response to the angular momentum gained or lost by the gas and the evolution of the gravitational potential as mass accumulates in the center.

\section{Flows in $3 D$}

Most 3D studies of gas flows in bars have employed the SPH method with an isothermal equation of state. Since there are few results from other 3D methods with which to compare, it makes sense to compare with the $2 \mathrm{D}$ behavior, especially as no dramatically new features have been reported that arise specifically from the freedom of motion in 3D. Indeed, Pérez (2008) showed that the flow velocities obtained by SPH compared well with those from a 2D Eulerian method.

Another feature of added realism in most studies is that the self-gravitating evolution of the SPH particles is combined with that of stellar particles (e.g. Berentzen et al., 1998); (Fux, 1999). This aspect therefore implies that the simulations capture both the angular momentum loss to the bar, and the changing gravitational potential as the gas accumulates in the center. However, while the flow patterns are broadly similar to those seen in 2D models, a characteristic new feature of many of these Lagrangian models is a high inflow rate of isothermal gas to the center. ${ }^{9}$ This finding raises a concern that the quantitative inflow rate may be substantially over-estimated because of the numerical viscosity inherent in the SPH method. An artifact of this kind will cause gas to accumulate in the center too quickly, and the effects of the central mass build-up, especially in gas-rich models, may occur too rapidly. Note that this concern is not over the physical process, which surely does happen, but over the rate at which it happens in the simulations.

\footnotetext{
9 Debattista et al. (2006) found reduced inflow with an adiabatic equation of state because the gas is then more resistant to compression. However, the pressure of even an isothermal gas may be unrealistic (see $I$ I.K).
} 
Berentzen et al. (2007) found that the build-up of a central mass concentration due to gas inflow caused a slight increase in the bar pattern speed, probably because the increased mass in the center raises orbit frequencies. They also found a reduced slow-down rate of the bar, since the bar must take up the angular momentum lost by the gas as it is driven inwards. Another finding was that a significant gas fraction altered the buckling behavior, which changed the final strength and 3D shape of the bar. In a follow-up study, Villa-Vargas et al. (2010) found that moderate fractions of gas $(\$ 5 \%$ of the disk mass) have little effect on the behavior; the bar grew, slowed and buckled pretty much as in a comparison stars only case.

\section{F. Bar dissolution}

Bars in $N$-body simulations that do not include any dissipative component or a live halo are long-lived structures (Miller \& Smith, 1979) that are also quite robust (Sparke \& Sellwood, 1987). But it has long been recognized (e.g. Pfenniger \& Norman, 1990) that the buildup of a central mass concentration (CMC) at the center of the bar can change its dynamical structure. Studies to determine the response of a bar to an imposed central mass (Norman et al., 1996); (Shen \& Sellwood, 2004); (Athanassoula et al., 2005); (Debattista et al., 2006) have generally found that the bar is weakened, but not completely destroyed by a central mass as large as a few percent of the disk mass, and still larger masses are needed to cause the bar to dissolve entirely. The destructive power of a given mass is also increased by making it more dense. Note that a high central density requires that simulation particle orbits near the center have short time steps (Shen \& Sellwood, 2004), and numerical errors in this regime can accelerate bar dissolution.

The CMC alters the gravitational potential of the bar, which in turn requires the orbital structure to adjust. Shen \& Sellwood (2004) found that the massive compact $\mathrm{CMC}$ in their model made large parts of phase space chaotic, ${ }^{10}$ causing an abrupt dissolution of the bar. Lower mass CMCs also caused some orbits to become chaotic, weakening the bar after which the weakened bar continued to adjust more gradually toward a new structure in the presence of the CMC.

A complete dissolution of the bar leaves the disk dynamically hot, since the highly eccentric orbits of the bar no longer remain aligned in a coherent streaming flow, but become randomly oriented. The process can be very rapid because of collective effects; the coherent alignment of the bar orbits is maintained by the bar potential and, as the bar weakens, the orbits of remaining stars are less

10 Gerhard \& Binnev (1985) predicted this consequence for nonrotating ellipsoidal galaxies. strongly constrained to precess at the original common rate. Since the bar has usually buckled by this time, the hot inner disk formed this way is also quite thick. Finally, a very dense central mass can scatter orbits in any direction, and the stars could take up a spheroidal shape, perhaps flattened slightly by the potential of the surrounding disk (Norman et al., 1996). There should be observable consequences from this sequence of events that could test the predictions of the simulations.

Bournaud et al. (2005) and Combes (2008), who use sticky particles to mimic gas, claim that the back reaction of the torque between the bar and the gas can be strong enough to dissolve the bar. They correctly point out that gas inflow must add angular momentum to the bar which should weaken it (Lynden-Bell \& Kalnajs, 1972). However, the angular momentum required to dissolve the bar should be at least roughly equal to that it lost to the outer disk when it was formed, perhaps more if the bar has been intensified through spiral activity or halo friction ( $\mathrm{D.C}$ ). Thus gas inflow through the comparatively small lever arm of the bar, in comparison to the outer disk, would indeed need to be prodigious to supply the angular momentum to unbind the bar. Berentzen et al. (2007), for example, did find that the bar weakened earlier as the gas mass fraction was increased, but they argued this behavior was caused by the accumulation of mass into the center rather than a back-reaction of the torque between the bar and the gas.

A third possible internal method to weaken a bar is an interaction with an exceptionally strong spiral, which has occurred in a few simulations (e.g. Sellwood \& Moore, 1999; Solway et al., 2012). Lynden-Bell \& Kalna is (1972) derived Eq. (20) by averaging over all phases, which they assumed to be uniformly populated, leading them to the widely cited conclusion that spirals remove angular momentum from the inner disk. However, the stars are far from uniformly distributed in azimuth near the end of a bar and the fact that they are trapped in the bar further invalidates, in this context, the assumptions that underlie the derivation of Eq. (20). The behavior in this more complex situation seems to depend on the relative phase of the spiral arm and the bar. For most of the cases when the bar leads, or is close to the same phase, as the inner end of the spiral, the spiral can remove angular momentum from nonbar stars which may allow them to become trapped into the bar, thereby increasing the bar strength, as described in \$V.C. On the other hand, when the spiral density maximum significantly leads the bar, their mutual attraction adds angular momentum to stars in the bar, which weakens it. This behavior has not been studied in detail, and further work is required to understand it and quantify its likelihood.

All three bar weakening mechanisms are discussed further in 


\section{G. Discussion of bar fraction}

None of the proposed methods to form bars, or of preventing their formation, seems able to give a convincing explanation for the observed fraction of bars in galaxies. Furthermore, Bosma (1996), Courteau et al. (2003), and others have pointed out that barred galaxies seem little different from their unbarred cousins in most respects - e.g. they lie on the same Tully-Fisher relation. Sánchez-Janssen \& Gadotti (2013) did find significant differences between barred and unbarred galaxies in photometric parameters, which they attribute to evolution caused by the bar.

Barazza et al. (2008) report an anticorrelation of bar frequency with the bulge light fraction and Buta et al. (2010a) found a decreased frequency of strong bars in S0 galaxies, which have dense and massive bulges. Both these studies offer weak support for the stabilizing mechanism proposed by Toomre (1981). But this cannot be the whole story because some near-bulgeless disks lack a strong bar (e.g. M33) while other barred disks have massive bulges.

The inability of theory or data to find a clear predictor for the incidence of a bar in a particular galaxy suggests that whether a particular galaxy is or is not barred may depend on unobservable factors such as its formation history (see also Sheth et al., 2012).

Erwin (2005, see also Hoyle et al. 2011) found that bars in early-type galaxies are larger than those in latetype galaxies, both in absolute size and in terms of the scale length of the disk light. He also noted that bars in many real galaxies, especially of late Hubble type, are shorter than those in simulations, which is another reason to think that our understanding of bar formation in real galaxies remains incomplete.

\section{H. Bar pattern speeds}

Tremaine \& Weinberg (1984a) devised a method to measure the pattern speed of a bar directly from observations of a tracer component, which must obey the equation of continuity. Their original method assumes that the galaxy has but a single pattern, and would yield a misleading result were there more than one pattern, each rotating at a different angular rate.

The stellar light distribution of early-type barred galaxies is believed to obey the equation of continuity because these galaxies have little dust obscuration and no star formation. They also rarely possess prominent spirals in the outer disk. Results of many studies using this method for early-type barred galaxies were summarized by Corsini (2008). While some individual measurements are quite uncertain, the data seem to favor $1<\mathcal{R} \lesssim 1.4$. Chemin \& Hernandez (2009) found a counter-example in a low-luminosity galaxy.

Fathi et al. (2009) and Meidt et al. (2009) applied the method of Tremaine \& Weinberg (1984a) to ionized and to molecular gas, respectively. Both groups argue that this is valid, even though the separate gas components do not obey the continuity equation that underlies the method. Fathi et al. (2009) generally found fast bars. Meidt et al. (2008) generalized the method to attempt to measure radial variations in the pattern speed and Meidt et al. (2009) found suggestions of pattern speeds that are lower at large radii than those near the center.

Other methods can yield indirect estimates of bar pattern speeds. Fits of models of the gas flow (\$V.E) have been reported for a few galaxies (Lindblad et al., 1996); (Weiner et al., 2001); (Pérez et al., 2004); (Zánmar Sánchez et al., 2008), finding $\mathcal{R} \sim 1.2$ in all cases. Athanassoula (1992) argued that the shapes and locations of dust lanes in bars also seem to suggest that $\mathcal{R} \simeq 1.2$. If the $4: 1$ resonant orbit family (dotted curves in Fig. 11) gives rise to the "boxy" appearance of a bar, then that bar must be fast, as the orbit family cannot be populated in slow bars. Identifying a ring in a barred galaxy as the location of a major resonance with the bar (Buta \& Combes, 1996) yields, with kinematic information, an estimate of the pattern speed.

Rautiainen et al. (2008) computed models of the stellar and gas (using sticky particles) responses to forcing by photometric models of 38 barred galaxies, in which they assumed that the entire nonaxisymmetric structure rotated at the same pattern speed. They attempted to match the model to the visual morphology of the galaxy, and found a range of values for $\mathcal{R}$. However, in most cases where $\mathcal{R} \gg 1$, the fit is dominated by the outer spiral, which may have a lower angular speed than does the bar.

\section{Bars within bars}

The nuclear regions of many barred galaxies show isophote twists (e.g. Shaw et al., 1993) that are interpreted as inner secondary bars within large-scale primary bars. Erwin \& Sparke (2002) identified secondary bars in $>25 \%$ of barred galaxies and reported that they have a length some $\sim 12 \%$ of that of the primary bar. The deprojected angles between the principal axes of the two bars appeared to be randomly distributed, suggesting that the two bars may tumble at differing rates. This inference was supported by Corsini et al. (2003), who used the Tremaine \& Weinberg (1984a) method to show that the two bars in NGC 2950 could not have the same rotation rates; Maciejewski (2006) used the same data to argue that the secondary bar has a large retrograde pattern speed. Fathi et al. (2007) infer an angular speed for the secondary bar that is higher than that of the primary in NGC 6946.

The theoretical challenge presented by these facts is substantial, and progress toward understanding the dynamics has been slow. Maciejewski \& Sparke (2000) studied the orbital structure in a potential containing two nonaxisymmetric components rotating at differing rates. 
However, a self-consistent secondary bar can neither rotate at a uniform rate (Louis \& Gerhard, 1988) nor can it maintain the same shape at all relative phases to the primary.

Friedli \& Martinet (1993) argued that gas was essential to forming secondary bars (see also e.g. Heller et al., 2001); (Englmaier \& Shlosman, 2004). However, some of the collisionless simulations reported by Rautiainen \& Salo (1999) and Rautiainen et al. (2002) manifested dynamically decoupled inner structures when the inner disk had high orbital frequencies due to a dense bulge. The structure was more spiral-like in some models, but others appeared to show inner bars that rotated more rapidly than the main bar.

Debattista \& Shen (2007) created long-lived, doublebarred galaxy models in collisionless $N$-body simulations having dense inner disks, which they described as pseudobulges. They followed up with a more detailed study (Shen \& Debattista, 2009) that also made some predictions for observational tests. The secondary bars in their models indeed rotated at nonuniform rates, with a shape that also varied systematically with phase relative to that of the primary.

These models prove that purely collisionless dynamical systems can support this behavior. However, it remains unclear what initial conditions have given rise to doublebarred galaxies in nature.

The possible consequence of gas inflow in these galaxies has attracted a lot of attention. Shlosman et al. (1989) speculated that bars within bars might lead to gas inflow over a wide range of scales, from global to the parsec scale where accretion onto a black hole might cause AGN activity. While inflows may have been observed (e.g. Haan et al., 2009) ; (van de Ven \& Fathi, 2010), understanding of gas flow in these nonsteady potentials remains rather preliminary (Maciejewski et al., 2002); (Heller et al., 2007).

\section{J. Fueling of AGN by bars?}

Many papers (a partial list is: Knapen et al., 2000); (Laine et al., 2002); (Laurikainen et al., 2004); (Hao et al., 2009); (Lee et al., 2012) have discussed the vexing question of whether there is, or is not, an excess of active galactic nuclei (AGN) in barred galaxies. Even the observational question is hard to answer, because a low level of AGN activity can be confused by a high rate of star formation, and low-ionization nuclear emission regions (LINERs). It is also necessary to ensure that the barred and unbarred galaxy samples to be compared have similar distributions of luminosities, colors, etc.

Emphatically one can answer that a single large-scale bar in a galaxy cannot drive gas close enough to the black hole to be accreted, and therefore produce an enhanced level of activity. Torques on the gas from the bar are able to reduce its angular momentum by about a single order of magnitude, leaving it orbiting the nucleus at speeds $\gtrsim 100 \mathrm{~km} \mathrm{~s}^{-1}$ at a distance $\gtrsim 200 \mathrm{pc}$. Its angular momentum must be reduced by at least a further 2 orders of magnitude before the gas could join even the dusty torus that is thought to surround the accretion disk in a typical AGN (Krolik, 1999). Thus the essence of the debate is whether secondary (or even multiple) bars, nuclear spirals, magnetic fields, etc. can bridge this gap and deliver to the accretion disk some of the larger supply of circum-nuclear gas that resides in barred galaxies.

Since the argument over the observational evidence continues, with perhaps the nay sayers in the ascendant at the present time, one concludes that there is no clear, direct connection between large-scale bar inflow and AGN activity, and there may be none at all.

\section{DYNAMICAL FRICTION}

Chandrasekhar (1943) pointed out that a massive object moving through a background sea of light particles would experience a drag force, known as dynamical friction. It is believed to affect globular clusters, satellite galaxies, and bars as they move or rotate inside dark matter halos. Orbital decay of satellites, or the slowdown of bars, together with the gain of energy by the halo, are important aspects of secular evolution.

\section{A. Mechanism}

Each particle in the background sea experiences an attractive gravitational impulse as it is passed by the advancing massive body. Since the attracted particles converge behind the perturber as it moves forward, the perturber in effect "focuses" the background particles into a trailing density excess, or wake. The gravitational attraction between the wake and the perturber gives rise to an apparent "frictional" drag that slows the motion of the perturber. The kinetic energy lost by the perturber is added to the random motion of the background particles.

The deceleration of a mass $M$, moving at speed $v_{M}$, through a background sea of particles of uniform density $\rho$ with an isotropic velocity spread $\sigma$ may be written (BT08, §8.1)

$$
\frac{d v_{M}}{d t}=4 \pi \ln \Lambda G^{2} \frac{M \rho}{\sigma^{2}} V\left(\frac{v_{M}}{\sigma}\right),
$$

where $\ln \Lambda$ is the Coulomb logarithm introduced in $₫$ II.B. The function $V(x)$ describes how the retarding acceleration varies with the speed of the perturber relative to the random motions of the stars, and is illustrated for Maxwellian velocity distribution in Fig. 12. A rapidly moving perturber gives weak impulses to the background particles that create a mild wake far downstream behind the perturber, and the drag therefore dies away as $V_{M} \rightarrow \infty$. The drag on a perturber moving slowly with respect to the rms motions of the particles is also mild, because there is only a small excess of particles dragging 


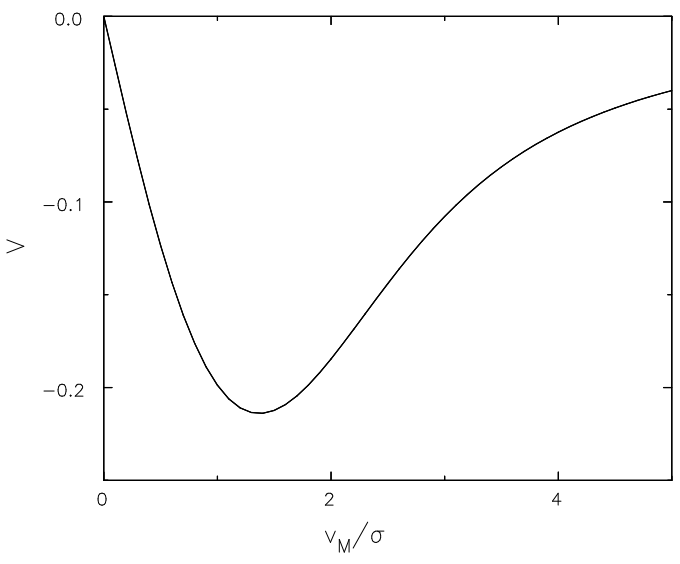

FIG. 12 The dimensionless acceleration function $V$ defined in Eq. (29) for the case of a Maxwellian distribution of velocities among the background particles. The function is negative because the acceleration is directed oppositely to the velocity.

it backward over those urging it forward, and the acceleration must also vanish as $V_{M} \rightarrow 0$. For a Maxwellian velocity distribution, the drag force peaks when the perturber moves somewhat faster than the $1 \mathrm{D}$ dispersion $\sigma$, i.e. the rms velocity in any coordinate direction.

\section{Improved treatment}

There are a number of conceptual problems with Chandrasekhar's analysis. Foremost is that fact that the hypothesized infinite sea of uniform density cannot be realized; away from the center of any finite distribution of matter, the motion of the perturber would not be a straight line but a curved orbit within the host mass distribution. Second, each particle in the sea is supposed to have a single interaction with the perturber, but particles must be bound to any finite system (otherwise it would disperse), and therefore repeatedly interact with a bound perturber. Choosing values for the density $\rho$ and velocity dispersion $\sigma$ appropriate for use in Eq. (29) also presents difficulties, especially when the mass distribution is highly inhomogeneous (Arena \& Bertin, 2007).

These problems were all overcome in a seminal paper by Tremaine \& Weinberg (1984b), who reformulated the analysis in action-angle variables. They found that the rate at which angular momentum is lost from a disturbing mass orbiting in a spherical system of background particles, hereafter a "halo," is given by an expression identical in form to that derived by Lynden-Bell \& Kalnais (1972) for a disk (Eq. 20 in the spherical case expresses the density in the $6 \mathrm{D}$ phase space. The number of possible resonances is substantially increased, since they arise wherever combinations of the three separate frequencies (orbital, radial and vertical) for the unperturbed motion of background particles match the angular frequency of the perturbation. In a follow-up paper, Weinberg (1985) evaluated the frictional drag expected from a halo, modeled as a singular isothermal sphere, on a rotating bar, modeled a homogeneous prolate spheroid rotating about an axis in its equatorial plane. Assuming the bar also had the moment of inertia of the rigid spheroid, Weinberg found that in a dense halo, the pattern speed of a strong bar would decay with a half-life of a few of its initial rotation periods.

While the complicated LBK torque expression (Eq.20) is daunting, its physical meaning is very similar to that of Chandrasekhar's formula (Eq. 29). The drag force arises because, to second order, the halo builds up a density excess, or wake, behind the perturber, leading to a gain, on average, of angular momentum by the halo particles and a corresponding loss by the perturber. The lagging wake was illustrated for the case of a bar by Weinberg \& Katz (2007, their Fig. 1).

As Lynden-Bell \& Kalnajs (1972) showed for spiral perturbations, halo particles that are not in resonance also experience changes that average to zero and therefore the drag is caused only by resonant particles. Referring back to Eq. (20) again, the contribution to the drag from each resonance depends on the gradient of the halo particle DF across that resonance, and there is generally an excess of gainers over losers. But the gainers will depopulate the high-density side, and cross the resonance to the previously low-density side. Thus, were the pattern speed of the perturber to remain constant, the local gradient across the resonance would be reduced, and the system would adjust toward a balanced equilibrium in which the gradient in the DF in the immediate vicinity of the resonance had flattened. Then the imbalance of gainers over losers would be erased and friction would die away. This effect was explicitly demonstrated by Lin \& Tremaine (1983) for the case of an orbiting satellite, and will turn out to be important in 9 VI.B.1.

However, the motion of the perturber is affected by its loss of angular momentum. An orbiting mass clearly sinks inward, making its angular speed about the center rise. Because a bar is not a solid object with a well-defined moment of inertia, Tremaine \& Weinberg (1984b) were less certain about whether its pattern speed would increase or decrease as a result of angular momentum loss. However, simulations (Sellwood, 1980); (Debattista \& Sellwood, 1998, 2000); (Sellwood, 2003); (Athanassoula, 2003); (Valenzuela \& Klypin, 2003); (Hollev-Bockelmann et al., 2005); (Martinez-Valpuesta et al., 2006); (Villa-Vargas et al., 2009); (Minchev et al., 2012a) have always found that bars slow as they lose angular momentum.

The change in pattern speed of the perturber causes the resonances to sweep through phase space (Sellwood \& Debattista, 2006); (Dubinski et al., 2009), and therefore the gradients across resonances do not have time to flatten and, in general, friction persists as the perturber loses angular momentum, although exceptions have occurred (see VI.B.1). 
Lin \& Tremaine (1983), Bontekoe \& van Albada (1987), Arena \& Bertin (2007) and Jardel \& Sellwood (2009) for an orbiting satellite, and Sellwood (2006) for a rotating bar, showed that the dependence of the friction term on the perturber mass, its angular speed, and both the dispersion and density of the halo, were all as expected from Eq. (29). The inclusion of self-gravity of the halo causes a slightly larger density response, with a corresponding increase in the drag force, but does not appear to change the scaling.

\section{Bar-halo friction}

The case of a bar rotating in a halo has received a great deal of recent attention, as friction from a moderately dense halo slows the bar on a time-scale of a few rotations (Sellwood, 1980); (Weinberg, 1985). Bars also grow in length and strength (i.e. the quadrupole moment rises) as they are braked by friction.

While many resonances exist between the bar and halo orbits, Weinberg \& Katz (2007) stressed that frictional drag was dominated by a small number of resonances, of which the ILR was by far the most important. The consequence of flattening the gradient in the halo DF at this resonance has also affected simulation results (see §I.B.1).

The phase lag angle between the axis of the halo response and principal axis of a bar varies with the drag force (Debattista \& Sellwood, 2000); (Sellwood, 2006). Friction is weak at high angular speeds because the halo response is almost exactly perpendicular to the bar, it strengthens as the pattern slows reaching a maximum when the response is $\sim 45^{\circ}$ to the bar axis, and decreases to zero as the response becomes aligned with the bar, which generally happens before the bar is brought to a complete stop. Thus the system reaches a steady state in which the corotating halo response, which has been described as a halo bar (Athanassoula, 2007), is aligned with the bar in the disk. Since the response of orbits to a steadily rotating bar potential is a forced precession (see $\sqrt{\mathrm{V} . \mathrm{A}}$ ), it is no surprise that studies of halo orbits in barred simulations (Athanassoula, 2002, 2003); (Ceverino \& Klypin, 2007) have found a large fraction of resonant orbits.

It is possible that such trapped orbits are responsible for the asymmetric distribution of thick-disk and/or luminous halo stars in the Milky Way. A density excess of such stars that are only a few kiloparsec from the Sun in the first Galactic quadrant, with no counterpart in the fourth quadrant, has been reported most recently by Larsen et al. (2008). A similar asymmetry, that could be aligned with the bar in the disk, was also found by the same group (Parker et al., 2004) in stellar kinematics.

Debattista \& Sellwood (2000) experimented with rotating halos, finding that the frictional drag varies with the degree and sense of halo rotation. It is little changed in halos having mild rotation in the same sense as the disk, and is significantly weakened only by an unrealistic degree of halo rotation.

The loss of angular momentum from the bar to the halo slows the bar and allows it to continue to grow (Debattista \& Sellwood, 2000). Continued evolution of bars in moderately dense halos (Athanassoula \& Misiriotis, 2002); (Athanassoula, 2003); (Martinez-Valpuesta et al., 2006) can cause the bar to grow until it fills almost the entire disk, at which point corotation can be outside the disk. The unreasonably large bars, relative to the disk size, obtained in these simulations would appear to argue against even moderately dense inner halos for real galaxy disks.

The halo that absorbs the angular momentum, need not be just the dark matter halo; visible spheroidal components are also angular momentum sinks. Saha et al. (2012) found that an initially nonrotating classical bulge acquires angular momentum from a strong bar and becomes triaxial, corotating with the bar when angular momentum transfer is complete. The bulge streaming motions they report are quite large.

\section{B. Constraint on halo density}

While the above theory leads to the expectation that a halo must exert a strong frictional drag on a massive bar, fully self-consistent simulations were needed to show that a bar in a disk embedded in a dense halo slows to the point that $\mathcal{R}>$ 1.4 (Debattista \& Sellwood, 2000); (Weinberg \& Katz, 2002); (Sellwood, 2003); (Valenzuela \& Klypin, 2003); (O’Neill \& Dubinski, 2003); (Hollev-Bockelmann et al., 2005); (Villa-Vargas et al., 2009), which is inconsistent with most observed values.

The pattern speeds of strong bars are generally fast, in that $\mathcal{R} \sim 1.2$ (Eq. 28); the evidence is reviewed in V.H. Furthermore, the slow bar reported by Chemin \& Hernandez (2009) is in the low-surfacebrightness galaxy UGC 628 , which is believed to have a large DM fraction that should have slowed a strong bar.

The observationally accessible ratio $\mathcal{R}$ is not a direct measure of friction, since both the bar length and corotation radius can change independently, but halo friction has driven this ratio to a high value in all collisionless simulations in which a strong bar rotated in a dense halo. Since no convincing counter-examples of strong bars in dense halos remaining fast for long periods have been found (see $\$$ VI.B.1), the simulations clearly predict low density halos for the majority of strongly barred galaxies. This result is little changed in simulations with moderate fractions of gas (Villa-Vargas et al., 2010), but friction is weaker because bars are smaller and weaker in simulations with unreasonably large gas fractions. Obviously, the magnitude of the friction force varies with bar strength, and weak bars therefore experience little friction, e.g. simulation MHH2 by Athanassoula (2003). 
Unfortunately, this author does not give the crucial value of $\mathcal{R}$ for any of her simulations, limiting further interpretation of her many results.

Since strong bars experience fierce braking from moderately dense halos, Debattista \& Sellwood (1998, 2000) argued that bars in real galaxies can remain fast only if the central dark matter density is much lower than was predicted by galaxy formation models. The implication of their result led to the conclusion being examined very closely by others, but even though two serious issues were raised (see below), their conclusion still holds.

\section{Anomalously weak friction}

Valenzuela \& Klypin (2003) reported that the bar in a disk embedded in a cuspy halo rotated at almost its initial speed for quite some time, and did not experience strong braking, which was inconsistent with all other numerical results and with theory! Sellwood \& Debattista (2006) reproduced their anomalous result when they reran the exact same model, and were able to show that the reason for the absence of friction stemmed from a flattened gradient in the halo DF at the crucial ILR. While the halo DF had a density gradient $\partial f / \partial L_{z}$ that was negative everywhere initially, the halo DF was changed by interactions with the disk bar as it formed. As the disk bar buckled, its pattern speed increased, with the implication that the resonances returned to parts of phase space where the previous changes at those resonances had flattened the gradient in the DF. Since friction depends upon there being an excess of gainers over losers caused by a decreasing density across the resonance, the absence of a gradient allowed the bar to rotate without friction exactly as Lin \& Tremaine (1983) had shown for a sinking satellite. Strong friction resumed after a while, probably because mild braking at minor resonances eventually moved the ILR resonance into a region where the DF gradient was normal. Sellwood \& Debattista (2006) were also able to show that the near-frictionless state was fragile, and could not be expected to arise in nature, since tiny perturbations by passing low-mass satellites were sufficient to shake the halo out of its "meta-stable" state and to cause fierce friction to resume.

This type of behavior was the root cause of the stochastic variations found by Sellwood \& Debattista (2009). They reported that the evolution of the bar pattern speed in many different random realizations of the same (isolated) model galaxy varied widely because the halo DF was sculptured by the early disk evolution, which was stochastic. The chance absence of gradients in the DF at the dominant resonance inhibited friction for periods that varied widely from realization to realization. Stochastic behavior was unaffected by changes to the numerical parameters, even the particle number, and persisted when they used a tree code instead of a grid code. Thus a simulation of an isolated galaxy that shows weak friction between a strong bar and a dense halo for a short period (e.g. Klypin et al., 2009) is inconclusive; firm evidence for weak friction that contradicts all the theory and experimental evidence summarized above would require longer simulations that are repeated with different random realizations.

\section{Particle number}

Weinberg \& Katz (2007) claimed that simulations require immense numbers of particles to reproduce the correct frictional drag. Their argument stemmed from their recognition that most of the frictional drag arises from the gradient in the halo DF across a single resonance, the ILR. They argued that too few particles in this small region of phase space would prevent the bar from experiencing the correct drag. Their predicted symptoms of this deficiency would be stochastic variations in the drag force resulting from shot noise in the local distribution of particles that happened to be in the vicinity of the resonance. (It should be emphasized that they were discussing friction with an imposed rigid bar, which is separate from the possible stochastic variations due to disk evolution that were discussed in the previous paragraph.) They went on to estimate that simulations needed a hundred-fold increase in the number of particles, from $\sim 10^{6}$ to $\sim 10^{8}$, before this problem would be brought under control.

Sellwood (2008b) conducted a series of simulations of exactly the kind that Weinberg \& Katz (2007) envisaged (a rigid bar in a halo of massive particles) and found that only very small numbers of particles $\left(\lesssim 10^{4}\right)$ behaved stochastically in the manner predicted by Weinberg \& Katz (2007). Once $N \gtrsim 10^{5}$, the evolution of both the pattern speed and the halo mass profile was independent of $N$, and no new behavior emerged when the number of particles was increased to $N=10^{8}$.

The reason for the discrepancy with the predictions of Weinberg \& Katz (2007) is that the resonances are broadened by the time dependence of the bar pattern speed. Their estimates of particle number requirements used only the intrinsic width of the resonance due to the finite amplitude of the perturbation - in effect, they assumed a fixed pattern speed. A much larger fraction of the particles than these authors expected contribute to the friction when the pattern speed decreases rapidly due to frictional drag from the halo, and friction can be reliably reproduced in simulations of strong bars with $10^{5}$ to $10^{6}$ halo particles. Ceverino \& Klypin (2007) and Dubinski et al. (2009) came to similar conclusions.

\section{Change in halo density}

As noted in $₫$ II.A all collisionless cold dark matter models of halo formation predict a steep inner gradient to the halo density profile, whereas shallower density profiles seem to be required (e.g. Sellwood, 2009); (Kuzio de Narav \& Spekkens, 2011). Dynamical friction 
between the baryons and the dark matter halo has been proposed as a possible solution to this discrepancy.

Weinberg \& Katz (2002) and El-Zant et al. (2001) argue that dynamical friction between the halo and bars or gas clumps respectively could transfer enough energy to the dark matter halo to reduce its density in the inner parts of galaxies. Note that the mechanism invoked in these papers is somewhat distinct from galaxy formation models (Read \& Gilmore, 2005); (Governato et al., 2010); (Pontzen \& Governato, 2012) that invoke repeated changes to the gravitational potential as gas disks collect and then evaporate; since this latter mechanism is essentially part of current ideas for galaxy formation, I do not review it here.

\section{Halo density reduction by bars}

A number of authors have reported a mild reduction in halo density resulting from bar friction (Hernquist \& Weinberg, 1992); (Debattista \& Sellwood, 2000); (Athanassoula, 2003); (McMillan \& Dehnen, 2005), and both Holley-Bockelmann et al. (2005) and Sellwood (2008a) verified that the dominant changes occurred at the ILR. Simulations with rigid bars that are pinned to a center can suffer from a numerical artifact if the halo is allowed to become lop-sided (Sellwood, 2003); (McMillan \& Dehnen, 2005), resulting in an erroneously large angular momentum transfer.

A convenient measure of halo density is its mean value inside the radius where the circular speed has risen to half its peak value (Alam et al., 2002). Sellwood (2008a) found that moderate, rigid bars reduced this quantity by a few percent, as already noted, but an order of magnitude reduction required a bar of length $\sim 12-20 \mathrm{kpc}$, an axis ratio $a / b \gtrsim 3$, and a bar mass $\gtrsim 30 \%$ of the enclosed halo mass. In fact, the angular momentum given up by the bar in order to achieve this density reduction exceeded the likely store of angular momentum in a galaxy disk (McMillan \& Dehnen, 2005); (Sellwood, 2008a).

Furthermore, a real bar, formed say through instabilities in the disk, contracts as it loses angular momentum to the halo, since the sizes of the stellar orbits that make up the bar themselves shrink. The resulting increase in the central attraction causes the halo density to rise, an effect that can overwhelm the density reduction due to angular momentum changes (Sellwood, 2003); (Colín et al., 2006); (Dubinski et al., 2009).

\section{Halo density reduction by moving mass clumps}

El-Zant et al. (2001), and later Tonini et al. (2006), proposed that moving clumps of dense gas will also transfer energy to the DM halo through dynamical friction and lower its density. They envisaged that baryons would collect into clumps through the Jeans instability as galaxies are assembled and present somewhat simplified cal- culations of the consequences of energy loss to the halo through dynamical friction. The dynamical process is that of mass segregation, which is well known in other contexts, such as in globular clusters (e.g. Merritt et al., 2004), but requires much larger mass differences for evolution on an interesting time scale.

The idea has been tested in idealized $N$-body simulations in which the heavy mass clumps were modeled as softened point masses (e.g. Jardel \& Sellwood, 2009); (Goerdt et al., 2010); (Cole et al., 2011). Generally, lowmass clumps were found to be ineffective, because dynamical friction is too weak. However, the orbital decay of a really massive clump, about $1 \%$ of the virial mass of the halo, does transfer enough energy to the halo particles to effect a substantial reduction in its density, even as the heavy clump itself deepens the gravitational potential.

Ma \& Boylan-Kolchin (2004) used clumps which were themselves composed of particles that could therefore suffer tidal stripping, etc. They reported that the stripped particles remained roughly at the radii at which they were detached, and also found that significant reduction in the halo density was caused by only the heaviest clumps.

The proposed mechanism faces a number of challenges, however. The settling gas clumps are assumed to maintain their coherence for many dynamical crossing times without colliding with other clumps or being disrupted by tidal fields and/or star formation, for example. Romano-Díaz et al. (2008a) claimed that baryonic physics had precisely this effect in their galaxy formation simulations, but calculations (e.g. Kaufmann et al., 2006) of the masses of the condensing gas clumps suggest they range up to only $\sim 10^{6} M_{\odot}$, which is too small to experience strong friction. Larger clumps will probably reside in subhalos, which may get dragged in, but simulations with subclumps composed of particles (e.g. Ma \& Boylan-Kolchin, 2004) indicate that the DM halos of the subclumps will be stripped, and the stripped matter largely replaces any DM moved outwards in the main halo. Thus if dynamical friction is to accentuate the separation of the baryons from dark matter before the baryonic mass clumps in subhalos settle to the center, they must somehow be stripped efficiently of their dark matter without dissolving the gas clumps themselves.

Mashchenko et al. (2006, 2008) argue that the energy input to the halo, mediated by the motion of the mass clumps, can be boosted if the gas is stirred by the usual feedback from stellar winds and supernovae. A challenge for this mechanism is the difficulty of accelerating such massive gas clumps into coherent motion, since the highpressure material from the postulated energetic events will vent more easily along low-density paths, thereby relieving the pressure before the dense clumps gain much momentum (e.g. MacLow \& Ferrara, 1999). 


\section{RINGS AND OUTER LIGHT PROFILES}

Gas in nonaxisymmetric galaxies is driven inward inside corotation, and outwards at larger radii. This behavior contrasts with that of the stars ( $($ III.E); dissipation allows the gas to stay dynamically cool while experiencing large changes in $L_{z}$. Secular evolution of this type is believed to be responsible for the formation of most rings observed in galaxies. The faint outer light profiles of galaxies also manifest features, but their origin is less clearly attributable to secular evolution.

Encounters between galaxies are invoked to explain other types of galaxy rings (Lvnds \& Toomre, 1976); (Struck, 2010); (Eliche-Moral et al., 2011) or polar rings (e.g. Sparke et al., 2008), which are not, therefore, the result of secular evolution.

\section{A. Rings}

Long-lived perturbations, such as bars, can drive gas radially until the flow stalls at resonances where rings of star-forming gas build up. Buta (1995) identifies three types of ring: outer rings, inner rings, and nuclear rings, all of which are commonly found in barred galaxies, but some are known in unbarred galaxies also. Outer rings, which are divided into two subtypes depending on their elongation relative to the bar, are generally believed to occur at the OLR of the bar. Inner rings have mean radii that are about as large as the bar semi-major axis, while nuclear rings are deep inside the bar. The rings are thought to depart from circles because of the quadrupole field of the bar, and the distortion is enhanced by being located at a resonance.

Outer rings have been identified in the light profiles of 66 early-type barred galaxies by Erwin et al. (2008a), who found an occurrence rate (or a feature at the expected radius) in $35 \%$ of the cases. Buta et al. (2010b) are conducting an on-going search using their deep $3.6 \mu \mathrm{m}$ survey with Spitzer (dubbed $\mathrm{S}^{4} \mathrm{G}$ ) for additional outer rings, but are finding few new cases, perhaps because these features tend to be quite blue.

The outer, inner, and nuclear rings are widely believed to form though secular evolution in, mostly barred, galaxies. Buta \& Combes (1996) give a thorough review of rings and the theory of secular formation of rings, and though written some years ago, remains reasonably up-to-date as the subject has not advanced much since. A more recent review of the properties of such rings and their formation mechanisms was included in Kormendy \& Kennicutt (2004).

The gas in a nonaxisymmetric potential must shock when periodic orbits cross (see $\$ \mathrm{~V}$.E), causing an irreversible change to the orbital motion. The shock is generally offset from the potential minimum, resulting in an angular momentum exchange between the gas and the bar or spiral. The position of the shock relative to the potential minimum determines the sign of the exchange: gas loses angular momentum inside corotation, whereas it gains outside this resonance, since the gas flow relative to the wave is in the opposite sense. Thus gas is driven away from corotation until the flow stalls, at an OLR, or where the dominant orbit family switches orientation in the nuclear region of a strong bar. Two orbit families can support rings at the OLR; just inside the resonance, orbits are elongated perpendicular to the bar, whereas the elongation is parallel to the bar just outside that resonance. The early simulations by Schwarz (1981), which employed sticky particles, were able to produce rings of both orientations, and there is evidence for both types in real galaxies (see Buta \& Combes, 1996, for examples). More recent models for the formation of outer rings were presented by Bagley et al. (2009) as the response of collisionless test particles to bar forcing, and they also compare their models with rear-infrared images of galaxies.

Inner rings are believed to be located at the ultraharmonic resonance (UHR, see $\$$ II.C) of the bar, where the potential supports 4:1 orbits (dotted in Fig. 11). Buta \& Combes (1996) suggest that inflow from corotation stalls at the UHR to make this ring, which is perhaps consistent with the behavior also found in Schwarz's work (Simkin et al., 1980). There the ring is simply a pointy oval, a shape that is often found in real galaxies. However, if the 4:1 resonant family is responsible, it is somewhat surprising that such rings are not more boxy; perhaps the theoretical interpretation of inner ring formation deserves further study.

Subsequent to the review by Buta \& Combes (1996), most attention has focused on nuclear rings. Bars appear to be efficient at driving gas inwards until the flow stalls in a nuclear ring, as described in $\mathrm{V} . \mathrm{E}$. The gas concentrations in these nuclear rings appear to be forming stars at a prodigious rate (Hawarden et al., 1986); (Maoz et al., 2001); (Benedict et al., 2002); (Mazzuca et al., 2008, 2011).

\section{B. Outer light profiles}

While galaxy disks are frequently described as exponentials, few galaxies have light profiles that can be fitted with a single exponential over several length scales. The light profiles reported by Freeman (1970) did not extend to very faint light levels, by the standards of today. Yet he identified both type I profiles, which were good exponentials over the limited dynamic range of his data, and type II, in which the surface brightness of the inner disk rises less rapidly than the inward extrapolation of the outer exponential. Both these types have been found in modern, much deeper photometry (Pohlen et al., 2002); (Bland-Hawthorn et al., 2005); (Erwin et al., 2005, 2008a); (Hunter \& Elmegreen, 2006); (Pohlen \& Trujillo, 2006), which also revealed type III, in which the light profile at large radii declines less steeply than the inner exponential. Erwin et al. (2008b) found that type II profiles are more common in 
barred galaxies. The fraction having type III profiles rises to late Hubble types, but galaxy interactions also appear to play a role (Erwin et al., 2005).

The origin and significance of this variety of behavior is still not fully understood, and may be related to galaxy formation, environment, or starformation efficiency (Sánchez-Blázquez et al., 2009); (Martínez-Serrano et al., 2009). However, some aspects may be due to internal disk evolution (Debattista et al., 2006); (Folve et al., 2008); (Minchev et al., 2012a). Martín-Navarro et al. (2012) proposed that breaks might be phenomena related to a threshold in the star formation, while truncations are more likely a real drop in the stellar mass density of the disk associated with the maximum angular momentum of the stars. On the other hand Roškar et al. (2008a) and Muñoz-Mateos et al. (2013) suggested internal secular evolution may be the cause.

While Trujillo et al. (2009) assert that the extended type III disk in M94 is not a ring, they nevertheless suggest it could be formed by an outflow in the disk that was driven by a rotating oval distortion in the inner part of the disk. Also noteworthy is the suggestion by Roškar et al. (2008a) that the radial decline in the mean ages of disk stars, caused by inside-out disk formation, could be reversed in the far outer disk by the outward migration of older stars. An attempt to verify this prediction (Yoachim et al., 2012) met with mixed results, however.

\section{PSEUDOBULGES AND LENSES}

Classical bulges, which have $R^{1 / 4}$ light profiles, are not strongly flattened, and rotate rather slowly, are believed to have been formed from violent mergers of protogalactic fragments in the early stages of galaxy formation, as described in $\amalg . A$. Galaxy disks with an embedded classical bulge are presumed to have built-up subsequently through the usual process of dissipative gaseous in-fall.

However, it has become clear that many galaxies host bulges having quite different properties that are now described as a pseudobulges. They have more nearly exponential light distributions Andredakis \& Sanders, 1994); (Fisher \& Drory, 2008), exhibit quite a high degree of rotation that has a roughly cylindrical flow pattern in 3D (Kormendy \& Illingworth, 1982), and are generally flatter than are classical bulges. Kormendy \& Kennicutt (2004), updated in Kormendy (2012), gave a more detailed description of how a pseudobulge can be distinguished from a classical bulge.

The observed properties of pseudobulges strongly suggest a different formation mechanism and it seems highly likely that they formed through internal evolution from the disk (Kormendy \& Kennicutt, 2004), and that this evolution is more rapid in galaxies with a higher gas fraction (Kormendy, 2012). Their basic idea is that pseudobulge formation is mediated by a bar, which first forms and buckles, as described in $\sqrt{\mathrm{V}}$ and then dissolves into a dy- namically hot, but flattened and rotationally supported bulgelike structure.

Kormendy (2012) proposed that a CMC of both stars and gas causes the bar to dissolve and create a pseudobulge. Gas is indeed driven inward by bars ( and simulations $(\sqrt{\mathrm{V} . \mathrm{F}})$ show that bars dissolved by massive CMCs do indeed form thickened, rotationally supported, near axisymmetric structures that resemble pseudobulges. The mass fraction in the dense central concentrations required to cause the bar to dissolve entirely is very high (e.g. Debattista et al., 2006, their simulation NG5). The more concentrated the mass the more efficiently it destroys the bar (Shen \& Sellwood, 2004), but the mass required is far larger than that suggested for any supermassive black hole (e.g. Gültekin et al., 2009). Large gas concentrations spread over an area of few hundred parsecs in radius are observed (e.g. Sakamoto et al., 1999); (Sheth et al., 2005), but again are nowhere near massive enough.

However, the gas in the nuclear region forms stars at a vigorous rate ( $\mathrm{VII}$.A $)$, with presumably a significant fraction of the mass being locked into long-lived stars that are gravitationally bound to the region where they formed. Kormendy (2012) therefore proposed that the stars built up in the nuclear region over a protracted period, together with the gas, eventually reach the combined mass required to dissolve the bar. Kormendy developed this proposal at length in his review, to which I refer the intersted reader for the full picture. If his plausible idea is correct, it once again implies that significant secular evolution is mediated by the behavior of gas.

No simulation has yet tested this suggestion, however. Previous studies of bar dissolution have created the central mass rather quickly, giving the bar little time to adjust as the mass grows, and further simulations of more gradual growth are needed to confirm that dissolution can eventually occur. The numerical task is particularly challenging for several reasons: (a) The evolution must be followed for a long period while the orbit time-scales in the very center are short. (b) Gas would have to be accreted continuously to the bar region, and the subsequent inflow rate should not be exaggerated by numerical viscosity (\$V.E) . (c) The halo would need to modeled self-consistently to follow bar growth through dynamical friction (

Two other methods that might dissolve a bar were discussed in $\$$ V.F Bournaud et al. (2005) and Combes (2008) suggest that the angular momentum added to bars as they drive gas inwards can weaken or destroy them. While more work on this scenario is needed, the inflow requirements are severe, and the consequence would not be so different from the build-up of a CMC. It is also noteworthy that some possible interactions between a bar and a strong spiral can weaken or destroy the bar. The mechanism and the conditions under which this behavior can occur also require further study, but the process may prove useful in this context, especially as strong spirals are most likely to arise in gas-rich outer disks. 
Bars could also be destroyed in minor mergers, of course. But to make a pseudobulge, the perturber would have to be dense enough to not be tidally disrupted before reaching the bar, but not so massive as to destroy the cylindrical flow pattern and/or shallow inner radial light profile. This degree of fine-tuning makes the explanation seem untenable to account for the observed high frequency of galaxies that seem to host pseudobulges (Kormendy et al., 2010); (Fisher \& Drory, 2011).

Other mechanisms for pseudobulge formation have been proposed. Guedes et al. (2013) found that the pseudobulge in their simulations was formed at an early stage through mergers, although its subsequent development was still mediated by a bar. Okamoto (2013) argued for an early starburst origin. However, these ideas may be inconsistent with a broad range of ages among the stars of pseudobulges (Fisher et al., 2009).

Kormendy (2012) also highlighted the lens component seen in some barred galaxies, which he argues is the intermediate case in which the bar is dissolving, while a lens in an unbarred galaxy is a fully dissolved bar (see also Combes, 2008). He therefore suggested that bar dissolution could be gradual, else we would not observe many transition cases. More moderate mass concentrations do cause bars to weaken and to become more oval (\$.F), but no author has commented, as far as this reviewer is aware, that the weakened bar in a simulation inhabits a lenslike structure. Nevertheless, lenses are established features of galaxies that seem most likely to have been created through disk evolution. The fact that we do not yet have a satisfactory explanation for their origin is part of the reason why galaxy evolution remains so fascinating.

\section{CONCLUSIONS}

This review has been rather narrowly focused on the internal evolution of isolated disk galaxies. The environment surely does play a substantial role in galaxy evolution; it is probably responsible for warps, lop-sidedness, tidal bridges and tails, and a whole host of phenomena related to halo substructure, halo streams, galaxy transformations, dry mergers, etc., but broadening this review to include all, or even some, of these topics would have necessitated either a shallower treatment or a greatly increased length.

The internally driven evolution of galaxy disks would scarcely be of any interest if the disk were composed of stars alone. Spiral activity would heat the disk on the time-scale of a few disk rotations, causing later spiral episodes to be progressively weaker and less distinct. The extent to which the overall distribution of angular momentum among the stars could be rearranged on large scales is strongly limited, since redistributive changes necessarily increase random motion. The fractional change in angular momentum of a distribution of stars ( ical radial excursion $a \sim \sigma_{R} / \kappa$ is bounded by

$$
\left|\frac{\Delta L_{z}}{L_{z}}\right| \lesssim \frac{a}{R m} \frac{\sigma_{R}}{V_{c}},
$$

where $V_{c}$ is the circular orbit speed at radius $R$, and $m \gtrsim 2$ is the angular periodicity of the spiral patterns. Thus the small value of both factors on the right-hand side provides a very tight constraint on the extent to which the distribution of angular momentum among the stars of a galaxy disk can have changed since their birth.

However, this constraint does not apply to individual stars, which can migrate radially for large distances within the disk through interactions near the corotation resonance of spirals (\$II.C). Since gains by some stars are roughly matched by losses by others in every diffusive step, these changes alter only the distribution of metals in the disk with almost no change to its dynamical structure. In particular, they neither lead to increased random motion, nor do they cause the disk to spread.

Note also that Eq. (30) does not limit the possible angular momentum changes of the gas component. The random motions of gas clouds, which experience similar radial accelerations from nonaxisymmetric disturbances as do the stars, are quickly damped through dissipative collisions with other clouds. Furthermore, the low velocity dispersion of the clouds makes them highly responsive to nonaxisymmetric disturbances, allowing them to exchange angular momentum with the driving potential to a greater extent than for the stars. Thus secular evolution in galaxies is greatly accelerated by the gas component. Since gas is consumed by star formation, it requires constant replenishment, as is expected in hierarchical structure formation models (e.g. Gunn, 1982).

The rising velocity dispersion of disk stars with age is now thought (@II.B.6) to be driven by the combined influence of deflections away from circular orbits by scattering at the resonances of spiral patterns, with the resulting in-plane peculiar motions being efficiently redirected into the third dimension by encounters with massive gas cloud complexes. No other combination of heating and scattering can account for both the high dispersion of the older disk stars and the fact that the velocity ellipsoid maintains a roughly constant shape as it grows in size. This combination of factors has not been tested in fully selfconsistent simulations because particle masses in most simulations are too large to mimic the two processes separately. The vertical heating that has been reported in some simulations is probably due to collisional relaxation (Sellwood, 2013b).

Bars are another important agent of secular evolution. The formation of a bar causes the largest change in the distribution of angular momentum among the stars of a disk, and further evolution occurs only through the influence of the outer disk, halo, and/or gas component. Bars can continue to grow, losing angular momentum to the outer disk, or to the halo, and the fact that bars are usually surrounded by an extensive disk suggests that 
halos cannot be dense enough to cause them to grow excessively (\$V.C).

Bars slow, as well as grow, through dynamical friction from the halo (\$VI). The loss of angular momentum by this mechanism also causes the disk mass to contract slightly, which actually deepens the gravitational potential, overwhelming any tendency for halo density to decrease as a result of its energy gain from the disk. While the central density rises, bars also grow in length as they slow, and the fact that corotation of most bars today appears to lie just beyond the bar end requires that the inner DM halos have lower densities than is predicted by $\Lambda \mathrm{CDM}$ models of galaxy formation ( $\mathrm{VI}$. ).

Bars also drive gas in towards their centers, causing the build up of gas-rich nuclear rings (\$V.E) where stars are seen to form at a high rate ( inflow over the lifetime of a galaxy can lead to the build up of concentrations of stars and gas in the center that may be able to destroy the bar and to form a pseudobulge ( (VIII).

Substantial evolutionary changes to the structure of disks could also occur through outside intervention, although the degree to which minor mergers could be important is again strongly constrained by data ( $(\mathrm{IV})$. The infrequency of classical bulges (Kormendy et al., 2010) places strong constraints on past merging activity, as does both the thinness of the main disks, and the absence of young stars in thick disks.

The realization that secular evolution is capable of rearranging the structure of disk galaxies from their initially endowed properties has been gradual. The topic was perhaps begun by Kormendy (1979), and it has gradually gained credence, largely through his constant advocacy. Despite the enormous progress described in this review, there are many areas where more work, such as the shaping of rotation curves (\$II.D) and the weakening of bars by spirals ( $\$$.F $)$, or even new ideas, such as to account for the observed fraction of galaxies that host bars (\$V.G) or the formation of double bars (\$V.I) or of lens components (\$VIII), are needed. Above all, we need better algorithms, with low numerical viscosity ( $\mathrm{V} . \mathrm{E}$ ), to capture the role of gas in more realistic manner - a need that is also recognized in galaxy formation.

\section{Acknowledgment}

I thank Tad Pryor, Michael Solway, and Ortwin Gerhard for helpful conversations, and the editor for his patience. Comments by an anonymous referee, Rok Roškar, Victor Debattista, James Binney, and especially by John Kormendy were extremely valuable. This work was supported in part by NSF Grants AST-1108977 and AST1211793.

\section{References}

Abadi, M. G., Navarro, J. F., Steinmetz, M. \& Eke, V. R. 2003, ApJ, 597, 21

Agertz, O., Teyssier, R. \& Moore, B. 2011, MNRAS, 410, 1391

Agertz, O., et al. 2007, MNRAS, 380. 963

Alam, S. M. K., Bullock, J. S. \& Weinberg, D. H. 2002, ApJ, $\mathbf{5 7 2}, 34$

Allende Prieto, C., et al. 2008, Astron. Nach., 329, 1018

Andredakis, Y. C. \& Sanders, R. H. 1994, MNRAS, 267, 283

Antoja, T., Valenzuela, O., Pichardo, B., Moreno, E.,

Figueras, F. \& Fernández, D. 2009, ApJL, 700, L78

Antoja, T., et al. 2011, MNRAS, 418, 1423

Antoja, T., et al. 2012, MNRAS, 426, L1

Araki, S. 1985, PhD. thesis, MIT.

Arena, S. E. \& Bertin, G. 2007, A\&A, 463, 921

Athanassoula, E. 1992, MNRAS, 259, 345

Athanassoula, E. 2002, ApJL, 569, L83

Athanassoula, E. 2003, MNRAS, 341, 1179

Athanassoula, E. 2005, MNRAS, 358, 1477

Athanassoula, E. 2007, MNRAS, 377, 1569

Athanassoula, E., Bosma, A. \& Papaioannou, S. 1987, A\&A, 179, 23

Athanassoula, E., Lambert, J. C. \& Dehnen, W. 2005, MNRAS, 363, 496

Athanassoula, E. \& Misiriotis, A. 2002, MNRAS, 330, 35

Athanassoula, E., et al. 1990, MNRAS, 245, 130

Athanassoula, E. \& Sellwood, J. A. 1986, MNRAS, 221, 213

Aumer, M. \& Binney, J. J. 2009, MNRAS, 397, 1286

Baba, J., Saitoh, T. R. \& Wada, K. 2013, ApJ, 763, 46

Bagley, M., Minchev, I. \& Quillen, A. C. 2009, MNRAS, 395, 537

Bahcall, J. N. \& Casertano, S. 1985, ApJL, 293, L7

Balbus, S. A. \& Hawley, J. F. 1998, Rev. Mod. Phys. , 70, 1

Baldry, I. K., Glazebrook, K., Brinkmann, J., Ivezić, Ž., Lupton, R. H., Nichol, R. C. \& Szalay \& A. S. 2004, ApJ, 600, 681

Barbanis, B. \& Woltjer, L. 1967, ApJ, 150, 461

Bardeen, J. M., Bond, J. R., Kaiser, N. \& Szalay, A. S. 1986, ApJ, 304, 15

Barnes, J. E. \& Hernquist, L. 1992, ARAA, 30, 705

Barazza, F. D., Jogee, S. \& Marinova, I. 2008, ApJ, 675, 194

Belokurov, V., et al. 2006, ApJL, 642, L137

Benedict, G. F., Howell, A., Jorgensen, I., Kenney, J. \& Smith, B. J. 2002, AJ, 123, 1411

Benjamin, R. A., et al. 2005, ApJ, 630, L149

Bensby, T. 2013, arXiv:1308.5191

Bensby, T., Feltzing, S., Lundström, I. \& Ilyin, I. 2005, A\&A, 433, 185

Bensby, T., Oey, M. S., Feltzing, S. \& Gustafsson, B. 2007, ApJL, 655, L89

Bensby, T., Alves-Brito, A., Oey, M. S., Yong, D. \& Meléndez, J. 2011, ApJL, 735 L46

Berentzen, I., Athanassoula, E., Heller, C. H. \& Fricke, K. J. 2003, MNRAS, 341, 343

Berentzen, I., Heller, C. H., Shlosman, I. \& Fricke, K. 1998, MNRAS, 300, 49

Berentzen, I., Shlosman, I., Martinez-Valpuesta, I. \& Heller, C. H. 2007, ApJ, 666, 189

Bershady, M. A., Martinsson, T. P. K., Verheijen, M. A. W., Westfall, K. B., Andersen, D. R. \& Swaters, R. A. 2011, ApJL, 739, L47 
Bertin, G. \& Lin, C. C. 1996, Spiral Structure in Galaxies (Cambridge, MA: The MIT Press)

Bertin, G., Lin, C. C., Lowe, S. A. \& Thurstans, R. P. 1989, ApJ, 338, 78

Binney, J. J. 2010, MNRAS, 401, 2318

Binney, J. J. \& Lacey, C. G. 1988, MNRAS, 230, 597

Binney, J. \& Tremaine, S. 2008, Galactic Dynamics. Princeton Univ. Press, Princeton (BT08)

Bird, J. C., Kazantzidis, S. \& Weinberg, D. H. 2012, MNRAS, 420, 913

Bird, J. C., Kazantzidis, S., Weinberg, D. H., Guedes, J., Callegari, S., Mayer, L. \& Madau, P. 2013, ApJ, 773, 43

Bland-Hawthorn, J., Krumholz, M. R. \& Freeman, K. 2010, ApJ, 713, 166

Bland-Hawthorn, J., et al. 2005, ApJ, 629, 239

Blitz, L. \& Spergel, D. N. 1991, ApJ, 379, 631

Block, D. L. \& Wainscoat, R. J. 1991, Nature, 353, 48

Bontekoe, Tj. R. \& van Albada, T. S. 1987, MNRAS, 224, 349

Bosma, A. 1996, in IAU Colloq. 157, Barred Galaxies, ed. R. Buta, D. A. Crocker \& B. G. Elmegreen (San Francisco: ASP Conf series 91), 132

Bottema, R. 1993, A\&A, 275, 16

Bournaud, F. \& Combes, F. 2002, A\&A, 392, 83

Bournaud, F., Combes, F. \& Semelin, B. 2005, MNRAS, 364, L18

Bournaud, F., Elmegreen, B. G. \& Martig, M. 2009, ApJ, 707, L1

Bovy, J., Hogg, D. W. \& Roweis, S. T. 2009, ApJ, 700, 1794

Bovy, J., Rix, H-W. \& Hogg, D. W. 2012a, ApJ, 751, 131

Bovy, J., Rix, H-W., Liu, C., Hogg, D. W., Beers, T. C. \& Lee, Y. S. 2012b, ApJ, 753, 148

Boylan-Kolchin, M., Bullock, J. S. \& Kaplinghat, M. 2012, MNRAS, 422, 1203

Brook, C. B., Kawata, D., Gibson, B. K. \& Freeman, K. C. 2004, ApJ, 612, 894

Brook, C. B., Gibson, B. K., Martel, H. \& Kawata, D. 2005, ApJ, 630, 298

Brunetti, M., Chiappini, C. \& Pfenniger, D. 2011, A\&A, 534, A75

Bullock, J. S., Kolatt, T. S., Sigad, Y., Somerville, R. S., Kravtsov, A. V., Klypin, A. A., Primack, J. R. \& Dekel, A. 2001, MNRAS, 321, 559

Bureau, M. \& Athanassoula, E. 2005, ApJ, 626, 159

Burstein, D. 1979, ApJ, 234, 829

Buta, R. 1995, ApJS, 96, 39

Buta, R. \& Combes, F. 1996, Fund. Cosmic Phys., 17, 95

Buta, R. J., et al. 2009, AJ, 137, 4487

Buta, R., et al. 2010a, ApJ, 721, 259

Buta, R. J., et al. 2010b, ApJS, 190, 147

Byrd, G. G., Valtonen, M. J., Valtaoja, L. \& Sundelius, B. 1986, A\&A, 166, 75

Cameron, E., et al. 2010, MNRAS, 409, 346

Carlberg, R. G. 1987, ApJ, 322, 59

Carlberg, R. G. \& Freedman, W. L. 1985, ApJ, 298, 486

Carlberg, R. G. \& Sellwood, J. A. 1985, ApJ, 292, 79

Carollo, C. M., Stiavelli, M. \& Mack, J. 1998, AJ, 116, 68

Casagrande, L., Schönrich, R., Asplund, M., Cassisi, S., Ramirez, I., Meléndez, J., Bensby, T. \& Feltzing, S. 2011, A\&A, 530, A138

Casetti-Dinescu, D. I., et al. 2011, ApJ, 728, 7

Ceverino, D. \& Klypin, A. 2007, MNRAS, 379, 1155

Chakrabarty, D. 2007, A\&A, 467, 145

Chandrasekhar, S. 1943, ApJ, 97, 255
Chandrasekhar, S. 1987, Ellipsoidal Figures of Equilibrium, Dover: New York.

Chemin, L., et al. 2006, MNRAS, 366, 812

Chemin, L. \& Hernandez, O. 2009, A\&A, 499, L25

Cheng, J. Y., et al. 2012, ApJ, 752, 51

Chiba, M. \& Beers, T. C. 2000, AJ, 119, 2843

Chirikov, B. V. 1979, Phys. Rep., 52, 265-379

Christodoulou, D. M., Shlosman, I. \& Tohline, J. E. 1995, ApJ, 443, 551

Cole, D. R., Dehnen, W. \& Wilkinson, M. I. 2011, MNRAS, 416, 1118

Colín, P., Valenzuela, O. \& Klypin, A. 2006, ApJ, 644, 687

Combes, F. 2008, arXiv:0811.0153

Combes, F., Debbasch, F., Friedli, D. \& Pfenniger, D. 1990, A\&A, 233, 82

Combes, F. \& Sanders, R. H. 1981, A\&A, 96, 164

Comerón, S., et al. 2011, ApJ, 741, 28

Contopoulos, G. 1980, A\&A, 81, 198

Contopoulos, G. \& Papayannopoulos, Th. 1980, A\&A, 92, 33

Corder, S., Sheth, K., Scoville, N. Z., Koda, J., Vogel, S. N. \& Ostriker, E. 2008, ApJ, 689, 148

Corsini, E. M. 2008, in Formation and Evolution of Galaxy Bulges, IAU Symp. 245 (Dordrecht: Kluwer) p. 125

Corsini, E. M., Debattista, V. P. \& Aguerri, J. A. L. 2003, ApJL, 599, L29

Courteau, S., et al. 2003, ApJ, 594, 208

Curir, A., Mazzei, P. \& Murante, G. 2006, A\&A, 447, 453

Debattista, V. P., et al. 2004, ApJL, 604, L93

Debattista, V. P., Mayer, L., Carollo, C. M., Moore, B., Wadsley, J. \& Quinn, T. 2006, ApJ, 645, 209

Debattista, V. P. \& Sellwood, J. A. 1998, ApJL, 493, L5

Debattista, V. P. \& Sellwood, J. A. 1999, ApJL, 513, L107

Debattista, V. P. \& Sellwood, J. A. 2000, ApJ, 543, 704

Debattista, V. P. \& Shen, J. A. 2007, ApJL, 654, L127

Dehnen, W. 1998, AJ, 115, 2384

Dehnen, W. 2000, AJ, 119, 800

Dekker, E. 1976, Phys. Rep., 24, 315

Deng, L-C., et al. 2012, RAA, 12, 735

De Simone, R. S., Wu, X. \& Tremaine, S. 2004, MNRAS, 350, 627

Dickey, J. M., Hanson, M. M. \& Helou, G. 1990, ApJ, 352 522

Dierickx, M., Klement, R., Rix, H-W. \& Liu, C. 2010, ApJL, 725, L186

D’Onghia, E., Vogelsberger, M. \& Hernquist, L. 2013, ApJ, 766, 34

Drury, L. O'C. 1980, MNRAS, 193, 337

Dubinski, J., Berentzen, I. \& Shlosman, I. 2009, ApJ, 697, 293

Edvardsson, B., Andersen, B., Gustafsson, B., Lambert, D. L., Nissen, P. E. \& Tomkin, J. 1993, A\&A, 275, 101

Efremov, Yu. N. 2010, MNRAS, 405, 1531

Efstathiou, G., Lake, G. \& Negroponte, J. 1982, MNRAS, 199, 1069

Eliche-Moral, M. C., González-Garca, A. C., Balcells, M., Aguerri, J. A. L., Gallego, J., Zamorano, J. \& Prieto, M. 2011, A\&A, 533, A104

Elmegreen, D. M. \& Elmegreen, B. G. 1982, MNRAS, 201, 1021

Elmegreen, B. 1996, in IAU Colloq. 157, Barred Galaxies, ed. R. Buta, D. A. Crocker \& B. G. Elmegreen (San Francisco: ASP Conf series 91), 197

El-Zant, A., Shlosman, I. \& Hoffman, Y. 2001, ApJ, 560, 636

Englmaier, P. \& Shlosman, I. 2004, ApJL, 617, L115 
Erwin, P. 2005, MNRAS, 364, 283

Erwin, P., Beckman, J. E. \& Pohlen, M. 2005, ApJL, 626, L81

Erwin, P. \& Debattista, V. P. 2013, MNRAS, 431, 3060

Erwin, P., Pohlen, M. \& Beckman, J. E. 2008a, AJ, 135, 20

Erwin, P., Pohlen, M., Gutiérrez, L. \& Beckman, J. E. 2008b, in Formation and Evolution of Galaxy Disks, eds. J. G. Funes SJ \& E. M. Corsini (San Francisco: ASP) 396, p. 207

Erwin, P. \& Sparke, L. S. 2002, AJ, 124, 65

Eskridge, P. B., et al. 2000, AJ, 119, 536.

Evans, N. W. \& Read, J. C. A. 1998, MNRAS, 300, 106

Famaey, B., Pont, F., Luri, X., Udry, S., Mayor, M. \& Jorrissen, A. 2007, A\&A, 461, 957

Fathi, K., Toonen, S., Falcón-Barroso, J., Beckman, J. E., Hernandez, O., Daigle, O., Carignan, C. \& de Zeeuw, T. 2007. ApJL, 667, L137

Fathi, K., Beckman, J. E., Piñol-Ferrer, N., Hernandez, O., Martínez-Valpuesta, I. \& Carignan, C. 2009, ApJ, 704, 1657

Fisher, D. B. \& Drory, N. 2008, AJ, 136, 773

Fisher, D. B. \& Drory, N. 2011, ApJL, 733, L47

Fisher, D. B., Drory, N. \& Fabricius, M. H. 2009, ApJ, 697, 630

Foyle, K., Courteau, S. \& Thacker, R. J. 2008, MNRAS, 386, 1821

Fraternali, F. \& Tomassetti, M. 2012, MNRAS, 426, 2166

Freeman, K. C. 1970, ApJ, 160, 811

Freeman, K., et al. 2013, MNRAS, 428, 3660

Frenk, C. S. \& White, S. D. M. 2012, Ann. der Phys., 524, 507

Fridman, A. M. \& Polyachenko, V. L. 1984. Physics of Gravitating Systems (New York: Springer-Verlag)

Friedli, D., Benz, W. \& Kennicutt, R. 1994, ApJL, 430, L105

Friedli, D. \& Martinet, L. 1993, A\&A, 277, 27

Friedli, D. \& Pfenniger, D. 1990, in "Bulges of Galaxies", eds. B. J. Jarvis \& D. M. Terndrup (Garching: ESO workshop 35) p. 265

Fuchs, B., Dettbarn, C. \& Tsuchiya, T. 2005, A\&A, 444, 1

Fuhrmann, K. 2008, MNRAS, 384, 173

Fujii, M. S., Baba, J., Saitoh, T. R., Makino, J., Kokubo, E. \& Wada, K. 2011, ApJ, 730, 109

Fux, R. 1999, A\&A, 345, 787

Gadotti, D. A. 2011, MNRAS, 415, 3308

Gadotti, D. A., Athanassoula, E., Carrasco, L., Bosma, A., de Souza, R. E. \& Recillas, E. 2007, MNRAS, 381, 943

Gao, L., Frenk, C. S., Boylan-Kolchin, M., Jenkins, A., Springel, V. \& White, S. D. M. 2011, MNRAS, 410, 2309

Garcia-Barreto, J. A., Downes, D., Combes, F., Gerin, M., Carrasco, L. \& Cruz-Gonzales, I. 1991b, A\&A, 252, 19

Garcia-Barreto, J. A., Downes, D., Combes, F., Gerin, M., Magri, C., Carrasco, L. \& Cruz-Gonzalez, I. 1991a, A\&A, 244, 257

Gerhard, O. E. \& Binney, J. 1985, MNRAS, 216, 467

Gerhard, O. E. \& Martinez-Valpuesta, I. 2012, ApJL, 744, L8

Gerin, M., Combes, F. \& Athanassoula, E. 1990, A\&A, 230, 37

Gerin, M., Combes, F. \& Nakai, N. 1988, A\&A, 203, 44-50

Gerssen, J., Kuijken, K. \& Merrifield, G. K. 2000, MNRAS, 317, 545

Gerssen, J. \& Shapiro, M. R. 2012, MNRAS, 423, 2726

Gilmore, G. \& Reid, N. 1983, MNRAS, 202, 1025

Gnedin, O. Y., Goodman, J. \& Frei, Z. 1995, AJ, 110, 1105

Goerdt, T., Moore, B., Read, J. I. \& Stadel, J. 2010, ApJ,
725, 1707

Goldreich, P. \& Lynden-Bell, D. 1965, MNRAS, 130, 125

González-Fernández, C., López-Corredoira, M., Amôres, E. B., Minniti, D., Lucas, P. \& Toledo, I. 2012, A\&A, 546, A107

Governato, F., et al. 2009, MNRAS, 398, 312

Governato, F. et al. 2010, Nature, 463, 203

Grand, R. J. J., Kawata, D. \& Cropper, M. 2012, MNRAS, 421, 1529

Gratier, P., et al. 2010, A\&A. 522, A3

Grosbøl, P., Patsis, P. A. \& Pompei, E. 2004, A\&A, 423, 849

Guedes, J., Mayer, L., Carollo, M. \& Madau, P. 2013, ApJ, $\mathbf{7 7 2}, 36$

Gültekin, K., et al. 2009, ApJ, 698, 198

Gunn, J. E. 1982, in Astrophysical Cosmology, eds. H. A. Brück, G. V. Coyne \& M. S. Longair (Vatican City: Pontificia Academia Scientiarum) p. 233

Haan, S., Schinnerer, E., Emsellem, E., Garca-Burillo, S., Combes, F., Mundell, C. G. \& Rix, H.-W. 2009, ApJ, 692, 1623

Hahn, C. H., Sellwood, J. A. \& Pryor, C. 2011, MNRAS, 418, 2459

Hänninen, J. \& Flynn, C. 2002, MNRAS, 337, 731

Hao, L., Jogee, S., Barazza, F. D., Marinova, I. \& Shen, J. 2009, in ASP Conf. Ser. 419, Galaxy Evolution: Emerging Insights and Future Challenges, ed. S. Jogee, et al. (San Francisco, CA: ASP), 402

Hawarden, T. G., Mountain, C. M., Leggett, S. K. \& Puxley, P. J. 1986, MNRAS, 221, 41P

Haywood, M. 2008, MNRAS, 388, 1175

Haywood, M. 2012, in Assembling the Puzzle of the Milky Way Eds. C. Reylé, A. Robin \& M. Schultheis (EPJ Web of Conferences, 19) id.05001

Heller, C. H., Shlosman, I. \& Athanassoula, E. 2007, ApJ, 657, L65

Heller, C., Shlosman, I. \& Englmaier, P. 2001, ApJ, 553, 661

Hénon, M. 1973, in Dynamical Structure and Evolution of Stellar Systems, ed. L. Martinet \& M. Mayor (Sauverny: Geneva Observatory) p. 182

Hernandez, O., Carignan, C., Amram, P., Chemin, L. \& Daigle, O. 2005, MNRAS, 360, 1201

Hernquist, L. \& Weinberg, M. D. 1992, ApJ, 400, 80

Herrmann, K. A. \& Ciardullo, R. 2009, ApJ, 705, 1686

Hill, V., Babusiaux, C., Gmez, A., Haywood, M., Katz, D. \& Royer, F. 2012, in Assembling the Puzzle of the Milky Way Eds. C. Reylé, A. Robin \& M. Schultheis (EPJ Web of Conferences, 19) id.06001

Hinshaw, G., et al. 2013, ApJS, 208, 19

Hohl, F. 1971, ApJ, 168, 343

Hohl, F. 1973, ApJ, 184, 353

Holley-Bockelmann, K., Weinberg, M. \& Katz, N. 2005, MNRAS, 363, 991

Holmberg, J., Nordström, B. \& Andersen, J. 2007, A\&A, 475, 519

Holmberg, J., Nordström, B. \& Andersen, J. 2009, A\&A, 501, 941

Hopkins, P. F. 2013 MNRAS, 428, 2840

House, E. L., et al. 2011, MNRAS, 415, 2652

Hoyle, B., et al. 2011, MNRAS, 415, 3627

Huang, S. \& Carlberg, R. G. 1997, ApJ, 480, 503

Hunter, D. A. \& Elmegreen, B. G. 2006, ApJS, 162, 49

Ida, S., Kokuba, E. \& Makino, J. 1993, MNRAS, 263, 875

Ivezić, Ž. 2008, AJ, 684, 287

Jalali, M. A. 2007, ApJ, 669, 218 
James, R. A. \& Sellwood, J. A. 1978, MNRAS, 182, 331

Jardel, J. \& Sellwood, J. A. 2009, ApJ, 691, 1300

Jenkins, A. \& Binney, J. J. 1990, MNRAS, 245, 305

Julian, W. H. \& Toomre, A. 1966, ApJ, 146, 810

Jurić, M. 2008, AJ, 673, 864

Kalnajs, A. J. 1972, ApJ, 175, 63

Kalnajs, A. J. 1973, Proc. Astron. Soc. Australia, 2, 174

Kalnajs, A. J. 1976, ApJ, 205, 751

Kalnajs, A. J. 1977, ApJ, 212, 637

Kalnajs, A. J. 1978, in IAU Symposium 77 Structure and Properties of Nearby Galaxies eds. E. M. Berkhuisjen \& R. Wielebinski (Dordrecht:Reidel) p. 113

Kalnajs, A. J. 1983, in Internal Kinematics and Dynamics of Galaxies, IAU Symp. 100, ed. E. Athanassoula (Dordrecht: Reidel) p 87

Kalnajs, A. J. 1991, in Dynamics of Disc Galaxies, ed. B. Sundelius ( Gothenburg: Göteborgs University) p. 323

Kaufmann, T., Mayer, L., Wadsley, J., Stadel, J. \& Moore, B. 2006, MNRAS, 370, 1612

Kazantzidis, S., Zentner, A. R., Kravtsov, A. V., Bullock, J. S., \& Debattista, V. P. 2009, ApJ, 700, 1896

Kendall, S., Kennicutt, R. C. \& Clarke, C. 2011, MNRAS, 414, 538

Kent, S. M. 1986, AJ, 91, 1301

Kim, W-T., Seo, W-Y., Stone, J. M., Yoon, D. \& Teuben, P. J. 2012, ApJ, 747, 60

Kim, W-T. \& Stone, J. M. 2012, ApJ, 751, 124

Klypin, A., Valenzuela, O., Colín, P. \& Quinn, T. 2009, MNRAS, 398, 1027

Knapen, J. H., Shlosman, I. \& Peletier, R. F. 2000, ApJ, 529, 93

Koda, J., et al. 2009, ApJL, 700, L132

Kormendy, J. 1979, ApJ, 227, 714

Kormendy, J. 1983, ApJ, 275, 529

Kormendy, J. 2012, In XXIII Canary Islands Winter School of Astrophysics, "Secular Evolution of Galaxies" eds. J. Falcón-Barroso \& J. H. Knapen (Cambridge: Cambridge University Press) (to appear)

Kormendy, J., Drory, N., Bender, R. \& Cornell, M. E. 2010, ApJ, 723, 54

Kormendy, J. \& Illingworth, G. 1982, ApJ, 256, 460

Kormendy, J. \& Kennicutt, R. C. 2004, ARAA, 42, 603

Kormendy, J. \& Norman, C. A. 1979, ApJ, 233, 539

Kraljic, K., Bournaud, F. \& Martig, M. 2012, ApJ, 757, 60

Kravtsov, A. V., Klypin, A, \& Hoffman, Y. 2002, ApJ, 571, 563

Kregel, M., van der Kruit, P. C. \& de Grijs, R. 2002, MNRAS, 334, 646

Krolik, J. 1999, Active Galactic Nuclei. Princeton Univ. Press, Princeton (BT08)

Kroupa, P. 2002, MNRAS, 330, 707

Kulsrud, R. M., Mark, J. W-K. \& Caruso, A. 1971, Ap. Sp. Sci., 14, 52

Kuzio de Naray, R. \& Spekkens, K. 2011, ApJL, 741, L29

Lacey, C. G. 1984, MNRAS, 208, 687

Lacey, C. G. 1991, in Dynamics of Disc Galaxies, ed. B. Sundelius (Gothenburg: Göteborgs University) p. 257

Laine, S., Shlosman, I., Knapen, J. H. \& Peletier, R. F. 2002, ApJ, 567, 97

Larsen, J. A., Humphreys, R. M. \& Cabanela, J, E. 2008, ApJL, 687, L17

Larson, D., et al. 2011, ApJS, 192, 16

Laurikainen, E., Salo, H. \& Buta, R. 2004, ApJ, 607, 103

Lee, G-H., Woo, J-H., Lee, M. G., Hwang, H. S., Lee, J. C.,
Sohn, J. \& Lee, J. H. 2012, ApJ, 750, 141

Lee, Y. S. 2011, ApJ, 738, 187

Lewis, J. R. \& Freeman, K. C. 1989, AJ, 97, 139

Li, Z-Y. \& Shen, J. 2012, ApJL, 757, L7

Lin, D. N. C. \& Tremaine, S. 1983, ApJ, 264, 364

Lindblad, P. A. B., Lindblad, P. O. \& Athanassoula, E. 1996, A\&A, 313, 65

Little, B. \& Carlberg, R. G. 1991, MNRAS, 251, 227

Liu, C. \& van de Ven, G. 2012, MNRAS, 425, 2144

Liu, W. M. \& Chaboyer, B. 2000, ApJ, 544, 818

Loebman, S. R., Roškar, R., Debattista, V. P., Ivezić, Z̆., Quinn, T. R. \& Wadsley, J. 2011, ApJ, 737, 8

Louis, P. D. \& Gerhard, O. E. 1988, MNRAS, 233, 337

Lovelace, R. V. E. \& Hohlfeld, R. G. 1978, ApJ, 221, 51

Lütticke, R., Dettmar, R.-J. \& Pohlen, M. 2000, A\&A, 362, 435

Lynden-Bell, D. 1962, MNRAS, 124, 1

Lynden-Bell, D. 1963, The Observatory, 83, 23

Lynden-Bell, D. 1979, MNRAS, 187, 101

Lynden-Bell, D. \& Kalnajs, A. J. 1972, MNRAS, 157, 1

Lynds, R. \& Toomre, A. 1976, ApJ, 209, 382

Ma, C-P. \& Boylan-Kolchin, M. 2004, Phys. Rev. Lett. , 93, 21301

Maciejewski, W. 2006, MNRAS, 371, 451

Maciejewski, W. \& Sparke, L. S. 2000, MNRAS, 313, 745

Maciejewski, W., Teuben, P. J., Sparke, L. S. \& Stone, J. M. 2002, MNRAS, 329, 502

MacLow, M.-M. 1999, ApJ, 524, 169

MacLow, M.-M. \& Ferrara, A. 1999, ApJ, 513, 142

Majewski, S. R. 1993, ARAA, 31, 575

Mark, J. W-K. 1974, ApJ, 193, 539

Maoz, D., Barth, A. J., Ho, L. C., Sternberg, A. \& Filippenko, A. V. 2001, AJ, 121, 3048

Marinova, I. \& Jogee, S. 2007, ApJ, 659, 1176

Martín-Navarro, I., et al. 2012, MNRAS, 427, 1102

Martínez-Serrano, F. J., Serna, A., Doménech-Moral, M. \& Domínguez-Tenreiro, R. 2009, ApJL, 705, L133

Martinez-Valpuesta, I. \& Gerhard, 0. 2011, ApJL, 734, L20

Martinez-Valpuesta, I. \& Shlosman, I. 2004, ApJL, 613, L29

Martinez-Valpuesta, I., Shlosman, I. \& Heller, C. 2006, ApJ, 637, 214

Martini, P., Regan, M. W., Mulchaey, J. S. \& Pogge, R. W. 2003, ApJ, 589, 774

Mashchenko, S., Couchman, H. M. P. \& Wadsley, J. 2006, Nature, 442, 539

Mashchenko, S., Wadsley, J. \& Couchman, H. M. P. 2008, Science, 319, 174

Masset, F. \& Tagger, M. 1997, A\&A, 322, 442

Masters, K. L., et al. 2011, MNRAS, 411, 2026

Matsuda, T. \& Isaka, H. 1980, Prog. Theor. Phys., 64, 1265

Matteucci, F. \& Francois, P. 1989, MNRAS, 239, 885

Matthews, L. D. 2000, AJ, 120, 1764

Mayer, L. \& Wadsley, J. 2004, MNRAS, 347, 277

Mazzuca, L. M., Knapen, J. H., Veilleux, S. \& Regan, M. W. 2008, ApJS, 174, 337

Mazzuca, L. M., Swaters, R. A., Knapen, J. H. \& Veilleux, S. 2011, ApJ, 739, 104

McMillan, P. J. 2011, MNRAS, 418, 1565

McMillan, P. J. 2013, MNRAS, 430, 3276

McMillan, P. J. \& Binney, J. J. 2008, MNRAS, 390, 429

McMillan, P. J. \& Dehnen, W. 2005, MNRAS, 363, 1205

McMillan, P. J. \& Dehnen, W. 2007, MNRAS, 378, 541

McWilliam, A. \& Zoccali, M. 2010, ApJ, 724, 1491

Meidt, S. E., Rand, R. J., Merrifield, M. R., Debattista, V. 
P. \& Shen, J. 2008, ApJ, 676, 899

Meidt, S. E., Rand, R. J. \& Merrifield, M. R. 2009, ApJ, 702, 277

Méndez-Abreu, J., Sánchez-Janssen, R., Aguerri, J. A. L., Corsini, E. M. \& Zarattini, S. 2012, ApJL, 761, L6

Menéndez-Delmestre, K., Sheth, K., Schinnerer, E., Jarrett, T. H. \& Scoville, N. Z. 2007, ApJ, 657, 790

Merritt, D. \& Hernquist, L. 1991, ApJ, 376, 439

Merritt, D., Piatek, S., Portegies Zwart, S. \& Hemsendorf, M. 2004, ApJL, 608, L25

Miller, R. H. \& Smith, B. F. 1979, ApJ, 227, 785

Minchev, I., Boily, C., Siebert, A. \& Bienayme, O. 2010, MNRAS, 407, 2122

Minchev, I., Chiappini, C. \& Martig, M. 2013, A\&A, to appear (arXiv:1208.1506)

Minchev, I. \& Famaey, B. 2010, ApJ, 722, 112

Minchev, I., Famaey, B., Combes, F., Di Matteo, P., Mouhcine, M. \& Wozniak, H. 2011, A\&A, 527, A147

Minchev, I., Famaey, B., Quillen, A. C., Di Matteo, P., Combes, F., Vlaji, M., Erwin, P. \& Bland-Hawthorn, J. et al. 2012a, A\&A, 548, A126

Minchev, I., Famaey, B., Quillen, A. C., Dehnen, W., Martig, M. \& Siebert, A. 2012b, A\&A, 548, A127

Minchev, I. \& Quillen, A. C. 2006, MNRAS, 368, 623

Miwa, T. \& Noguchi, M. 1998, ApJ, 499, 149

Moster, B. P., Macciò, A. V., Somerville, R. S., Johansson, P. H. \& Naab, T. 2010, MNRAS, 403, 1009

Mould, J. 2005, AJ, 129, 698

Munn, J. A. 2004, AJ, 127, 3034

Muñoz-Mateos, J. C., et al. 2013, ApJ, 771, 59

Nataf, D. M., Udalski, A., Gould, A., Fouqué, P. \& Stanek, K. Z. 2010, ApJL, 721, L28

Ness, M., et al. 2012, ApJ, 756, 22

Nieten, Ch., Neininger, N., Guélin, M., Ungerechts, H., Lucas, R., Berkhuijsen, E. M., Beck, R. \& Wielebinski, R. 2006, A\&A, 453, 459

Nordström, B., Mayor, M., Andersen, J., Holmberg, J., Pont, F., Jørgensen, B. R., Olsen, E. H., Udry, S. \& Mowlavi, N. 2004, A\&A, 418, 989

Noguchi, M. 1987, MNRAS, 228, 635

Norman, C. A., Sellwood, J. A. \& Hasan, H. 1996, ApJ, 462, 114

Okamoto, T. 2013, MNRAS, 428, 718

O'Neill, J. K. \& Dubinski, J. 2003, MNRAS, 346, 251

Oort, J. H. 1962, in Interstellar Matter in Galaxies, ed. L. Woltjer (New York: Benjamin), p. 234

Ostriker, J. P. \& Peebles, P. J. E. 1973, ApJ, 186, 467

Palmer, P. L., Papaloizou, J. \& Allen, A. J. 1990, MNRAS, 243, 282

Palunas, P. \& Williams, T. B. 2000, AJ, 120, 2884

Parker, J. E., Humphreys, R. M. \& Beers, T. C. 2004, AJ, 127, 1567

Patsis, P. A., Skokos, Ch. \& Athanassoula, E. 2002, MNRAS, 337, 578

Pérez, I. 2008, A\&A, 478, 717

Pérez, I., Fux, R. \& Freeman, K. 2004, A\&A, 424, 799

Perlmutter, S., et al. 1999, ApJ, 517, 565

Perryman, M. A. C., et al. 2001, A\&A, 369, 339

Pfenniger, D. \& Friedli, D. 1991, A\&A, 252, 75

Pfenniger, D. \& Norman, C. 1990, ApJ, 363, 391

Piner, B. G., Stone, J. M. \& Teuben, P. J. 1995, ApJ, 449, 508

Pohlen, M., Dettmar, R.-J., Lütticke, R. \& Aronica, G. 2002, A\&A, 392, 807
Pohlen, M. \& Trujillo, I. 2006, A\&A, 454, 759

Polyachenko, E. V. 2004, MNRAS, 348, 345

Polyachenko, E. V. 2013, Astron. Lett., 39, 72

Pompéia 2011, MNRAS, 415, 1138

Pontzen, A. \& Governato, F. 2012, MNRAS, 421, 3464

Prendergast, K. H. 1962, in Interstellar Matter in Galaxies, ed. L. Woltjer (New York: Benjamin), p. 217

Prendergast, K. H. 1983, in Internal Kinematics and Dynamics of Galaxies, IAU Symp. 100, ed. E. Athanassoula (Dordrecht: Reidel) p 215

Puech, M., Hammer, F., Hopkins, P. F., Athanassoula, E., Flores, H., Rodrigues, M., Wang, J. L. \& Yang, Y. B. 2012, ApJ, 753, 128

Purcell, C. W., Kazantzidis, S. \& Bullock, J. S. 2009, ApJL, 694, L98

Quillen, A. C. 2003, AJ, 125, 785

Quillen, A. C., Frogel, J. A., Kenney, J. D. P., Pogge, R. W. \& Depoy, D. L. 1995, ApJ, 441, 549

Quillen, A. C. \& Garnett, D. R. 2001, in Galaxy Disks and Disk Galaxies.eds. J. G. Funes SJ \& E. M. Corsini (San Francisco: ASP) 230, p. 87

Quillen, A. C. \& Minchev, I. 2005, AJ, 130, 576

Quillen, A. C., Minchev, I., Bland-Hawthorn, J. \& Haywood, M. 2009, MNRAS, 397, 1599

Quinn, P. J. \& Goodman, J. 1986, ApJ, 309, 472

Quinn, P. J., Hernquist, L. \& Fullagar, D. P. 1993, ApJ, 403, 74

Rafikov, R. R. 2001, MNRAS, 323, 445

Raha, N., Sellwood, J. A., James, R. A. \& Kahn, F. D. 1991, Nature, 352, 411

Rautiainen, P. \& Salo, H. 1999, A\&A, 348, 737

Rautiainen, P., Salo, H. \& Laurikainen, E. 2002, MNRAS, 337, 1233

Rautiainen, P., Salo, H. \& Laurikainen, E. 2008, MNRAS, 388, 1803

Read, J. I. \& Gilmore, G. 2005, MNRAS, 356, 107

Read, J. I. \& Hayfield, T. 2012, MNRAS, 422, 3037

Read, J. I., Lake, G., Agertz, O. \& Debattista, V. P. 2008, MNRAS, 389, 1041

Reddy, B. E., Lambert, D. L. \& Allende Prieto, C. 2006, MNRAS, 367, 1329

Reese, A., Williams, T. B., Sellwood, J. A., Barnes, E. I. \& Powell, B. A. 2007, AJ, 133, 2846

Regan, M. W., et al. 2006, ApJ, 652, 1112

Reid, I. N., Turner, E. L., Turnbull, M. C., Mountain, M. \& Valenti, J. A. 2007, ApJ, 665, 767

Riess, A. G., et al. 1998, AJ, 116, 1009

Rix, H-W. \& Bovy, J. 2013, A\&A Rev., 21, 61

Robertson, B., Bullock, J. S., Cox, T. J., Di Matteo, T., Hernquist, L., Springel, V. \& Yoshida, N. et al., 2006, ApJ, 645, 986

Roca-Fàbrega, S., Valenzuela, O., Figueras, F., RomeroGómez, M., Velázquez, H., Antoja, T. \& Pichardo, B. 2013, MNRAS, 432, 2878

Rodionov, S. A. \& Sotnikova, N. Ya. 2013, MNRAS, 434, 2373

Romano-Díaz, E., Shlosman, I., Hoffman, Y. \& Heller C. 2008a, ApJL, 685, L105

Romano-Díaz, E., Shlosman, I., Heller C. \& Hoffman, Y. 2008b, ApJL, 687, L13

Romeo, A. B. 1992, MNRAS, 256, 307

Romeo, A. B. 1998, A\&A, 335, 922

Romeo, A. B. \& Wiegert, J. 2011, MNRAS, 416, 1191

Roškar, R., Debattista, V. P., Quinn, T. R., Stinson, G. S. \& 
Wadsley, J. 2008a, ApJL, 684, L79

Roškar, R., Debattista, V. P., Stinson, G. S., Quinn, T. R., Kaufmann, T. \& Wadsley, J. 2008b, ApJL, 675, L65

Roškar, R., Debattista, V. P. \& Loebman, S. R. 2013, MNRAS, 433, 976

Roškar, R., Debattista, V. P., Quinn, T. R. \& Wadsley, J. 2012, MNRAS, 426, 2089

Ruchti, G. R., et al. 2010, ApJL, 721, L92

Ruchti, G. R., et al. 2011, ApJ, 737, 9

Rybicki, G. B. 1972, in IAU Colloq. 10, Gravitational N-body Problem, ed. M. Lecar (Dordrecht: Reidel), 22

Saha, K., Martinez-Valpuesta, I. \& Gerhard, O. 2012, MNRAS, 421, 333

Sales, L. V., Helmi, A., Abadi, M. G., Brook, C. B., Gómez, F. A., Roškar, R., Debattista, V. P., House, E., Steinmetz, M. \& Villalobos, Á. 2009, MNRAS, 400, L61

Salo, H. 1991, A\&A, 243, 118

Sakamoto, K., Okamura, S. K., Ishizuki, S. \& Scoville, N. Z. 1999, ApJ, 525, 691

Samland, M. \& Gerhard, O. E. 2003, A\&A, 399, 961

Sánchez-Blázquez, P., Courty, S., Gibson, B. K. \& Brook, C. B. 2009, MNRAS, 398, 591

Sánchez-Janssen, R. \& Gadotti, D. 2013, MNRAS, 432, L56

Sandage, A. 1961, The Hubble Atlas of Galaxies, Carnegie Inst. of Washington.

Sanders, R. H. \& Huntley, J. M. 1976, ApJ, 209, 53

Sanders, R. H. \& Tubbs, A. D. 1980, ApJ, 235, 803

Scalo, J. \& Elmegreen, B. G. 2004, ARAA, 42, 275

Scannapieco, C., et al. 2011, MNRAS, 417, 154

Schinnerer, E., et al. 2013, ApJ, 779, 42

Schlesinger, K. J., et al. 2012, ApJ, 761, 160

Schönrich, R. \& Binney, J. 2009a, MNRAS, 396, 203

Schönrich, R. \& Binney, J. 2009b, MNRAS, 399, 1145

Schönrich, R. \& Binney, J. 2012, MNRAS, 419, 1546

Schönrich, R., Binney, J. \& Dehnen, W. 2010, MNRAS, 403, 829

Schwarz, M. P. 1981, ApJ, 247, 77

Schweizer, F. 1976, ApJS, 31, 313

Seabroke, G. M. \& Gilmore, G. 2007, MNRAS, 380, 1348

Sellwood, J. A. 1980, A\&A, 89, 296

Sellwood, J. A. 1981, A\&A, 99, 362

Sellwood, J. A. 1983, J. Comp. Phys., 50, 337

Sellwood, J. A. 1985, MNRAS, 217, 127

Sellwood, J. A. 1989a, in Dynamics of Astrophysical Discs, ed. J. A. Sellwood (Cambridge: Cambridge University Press) p. 155

Sellwood, J. A. 1989b, MNRAS, 238, 115

Sellwood, J. A. 1996, in IAU Symp. 169, Unsolved Problems of the Milky Way, ed. L. Blitz \& P. Teuben (Dordrecht: Kluwer) p. 31

Sellwood, J. A. 2000, Ap. Sp. Sci., 272, 31 (astro-ph/9909093)

Sellwood, J. A. 2003, ApJ, 587, 638

Sellwood, J. A. 2006, ApJ, 637, 567

Sellwood, J. A. 2008a, ApJ, 679, 379

Sellwood, J. A. 2008b, in Formation and Evolution of Galaxy Disks, eds. J. G. Funes SJ \& E. M. Corsini (San Francisco: ASP) 396, p. 341 (arXiv:0803.1574)

Sellwood, J. A. 2009, in IAU Symp. 254, The Galaxy Disk in Cosmological Context, ed. J. Andersen, J. BlandHawthorn \& B. Nordström, (Cambridge: Cambridge University Press) p. 73 (arXiv:0807.1973)

Sellwood, J. A. 2010, MNRAS, 409, 145

Sellwood, J. A. 2011, MNRAS, 410, 1637

Sellwood, J. A. 2012, ApJ, 751, 44
Sellwood, J. A. 2013a, in Planets Stars and Stellar Systems, v.5, eds. T. Oswalt \& G. Gilmore (Heidelberg: Springer) p. 923 (arXiv:1006.4855)

Sellwood, J. A. 2013b, ApJL, 769, L24

Sellwood, J. A. \& Balbus, S. A. 1999, ApJ, 511, 660

Sellwood, J. A. \& Binney, J. J. 2002, MNRAS, 336, 785

Sellwood, J. A. \& Carlberg, R. G. 1984, ApJ, 282, 61

Sellwood, J. A. \& Debattista, V. P. 2006, ApJ, 639, 868

Sellwood, J. A. \& Debattista, V. P. 2009, MNRAS, 398, 1279

Sellwood, J. A. \& Evans, N. W. 2001, ApJ, 546, 176

Sellwood, J. A. \& James, R. A. 1979, MNRAS, 187, 483

Sellwood, J. A. \& Kahn, F. D. 1991, MNRAS, 250, 278

Sellwood, J. A. \& Merritt, D. 1994, ApJ, 425, 530

Sellwood, J. A. \& Moore, E. M. 1999, ApJ, 510, 125

Sellwood, J. A., Nelson, R. D. \& Tremaine, S. 1998, ApJ, 506, 590

Sellwood, J. A. \& Sparke, L. S. 1988, MNRAS, 231, 25P

Sellwood, J. A. \& Wilkinson, A. 1993, Rep. Prog. Phys., 56, 173

Shaw, M. A., Combes, F., Axon, D. J. \& Wright, G. S. 1993, A\&A, 273, 31

Shen, J., Rich, R. M., Kormendy, J., Howard, C. D., De Propris, R. \& Kunder, A. 2010, ApJL, 720, L72

Shen, J. \& Debattista, V. P. 2009, ApJ, 690, 758

Shen, J. \& Sellwood, J. A. 2004, ApJ, 604, 614

Sheth, K., Melbourne, J., Elmegreen, D. M., Elmegreen, B. G., Athanassoula, E., Abraham, R. G. \& Weiner, B. J. 2012, ApJ, 758, 136

Sheth, K., Vogel, S. N., Regan, M. W., Thornley, M. D. \& Teuben, P. J. 2005, ApJ, 632, 217

Shetty, R., Vogel, S. N., Ostriker, E. C. \& Teuben, P. J. 2007, ApJ, 665, 1138

Shiidsuke, K. \& Ida, S. 1999, MNRAS, 307, 737

Shlosman, I., Frank, J. \& Begelman, M. C. 1989, Nature, 338, 45

Silk, J. \& Mamon, G. A. 2012, RAA, 12, 917

Simkin, S. M., Su, H. J. \& Schwarz, M. P. 1980, ApJ, 237, 404

Skibba, R. A., et al. 2012, MNRAS, 423, 1485

Skokos, Ch., Patsis, P. A. \& Athanassoula, E. 2002, MNRAS, 333, 847

Smith, M. C., Whiteoak, S. H. \& Evans, N. W. 2012, ApJ, 746, 181

Soderblom, D. R. 2010, ARAA, 48, 581

Sofue, Y. \& Rubin, V. 2001, ARAA, 39, 137

Solway, M., Sellwood, J. A. \& Schönrich, R. 2012, MNRAS, 422, 1363

Soubiran, C., Bienaymé, O., Mishenina, T. V. \& Kovtyukh, V. V. 2008, A\&A, 480, 91

Sparke, L. S. \& Sellwood, J. A. 1987, MNRAS, 225, 653

Sparke, L. S., van Moorsel, G., Erwin, P. \& Wehner, E. M. H. 2008, AJ, 135, 99

Spitzer, L. \& Schwarzschild, M. 1953, ApJ, 118, 106

Springel, V. 2010a, ARAA, 48, 391

Springel, V. 2010b, MNRAS, 401, 791

Springel, V., Frenk, C. S. \& White, S. D. M. 2006, Nature, 440, 1137

Sridhar, S. \& Touma, J. 1996, MNRAS, 279, 1263

Steinmetz, M., 2006, AJ, 132, 1645

Stone, J. M., Ostriker, E. C. \& Gammie, C. F. 1998, ApJL, 508, L99

Struck, C. 2010, MNRAS, 403, 1516

Skrutskie, M. F., et al. 2006, AJ, 131, 1163

Tagger, M., Sygnet, J. F., Athanassoula, E. \& Pellat, R. 1987, 
ApJL, 318, L43

Tamburro, D., Rix, H.-W., Leroy, A. K., Mac Low, M.-M., Walter, F., Kennicutt, R. C., Brinks, E. \& de Blok, W. J. G. 2009, AJ, 137, 4424

Tonini, C., Lapi, A. \& Salucci, P. 2006, ApJ, 649, 591

Toomre, A. 1964, ApJ, 139, 1217

Toomre, A. 1966, in Geophysical Fluid Dynamics, notes on the 1966 Summer Study Program at the Woods Hole Oceanographic Institution, ref. no. 66-46

Toomre, A. 1969, ApJ, 158, 899

Toomre, A. 1981, In "The Structure and Evolution of Normal Galaxies", Eds. S. M. Fall \& D. Lynden-Bell (Cambridge, Cambridge Univ. Press) p. 111

Toomre, A. 1983, in IAU Symposium 100, Internal Kinematics and Dynamics of Galaxies, ed. E. Athanassoula (Dordrecht: Reidel) p 177

Toomre, A. 1990, in Dynamics $\mathcal{E}$ Interactions of Galaxies, ed. R. Wielen (Berlin, Heidelberg: Springer-Verlag), p. 292

Toomre, A. \& Kalnajs, A. J. 1991, in Dynamics of Disc Galaxies, ed. B. Sundelius (Gothenburg: Göteborgs University) p. 341

Tóth, G. \& Ostriker, J. P. 1992, ApJ, 389, 5

Tremaine, S. \& Weinberg, M. D. 1984a, ApJL, 282, L5

Tremaine, S. \& Weinberg, M. D. 1984b, MNRAS, 209, 729

Trujillo, I., Martinez-Valpuesta, I., Martínez-Delgado, D. Peǹarrubia, J., Gabany, R. J. \& Pohlen, M. 2009, ApJ, 704, 618

Valenzuela, O. \& Klypin, A. 2003, MNRAS, 345, 406

van Albada, G. D. \& Roberts, W. W. 1981, ApJ, 246, 740

van der Kruit, P. C. \& Freeman, K. C 2011, ARAA, 49, 301

van der Kruit, P. C. \& Searle, L. 1981, A\&A, 95, 105

van de Ven, G. \& Fathi, K. 2010, ApJ, 723, 767

van Leeuwen, F. 2007, A\&A, 474, 653

Vásquez, S., et al. 2013, A\&A, 555, A91

Velazquez, H. \& White, S. D. M. 1999, MNRAS, 304, 254

Villalobos, Á. \& Helmi A. 2008, MNRAS, 391, 1806

Villalobos, Á, Kazantzidis, S. \& Helmi, A. 2010, ApJ, 718, 314

Villa-Vargas, J., Shlosman, I. \& Helller, C. 2009, ApJ, 707, 218
Villa-Vargas, J., Shlosman, I. \& Helller, C. 2010, ApJ, 719, 1470

Villumsen, J. V. 1985, ApJ, 290, 75

Visser, H. C. D. 1978, PhD. thesis, University of Groningen

Wada, K. 2004, In "Coevolution of Black Holes and Galaxies". Ed. L. C. Ho (Cambridge, Cambridge University Press) p. 186 (astro-ph/0308134)

Wada, K., Baba, J. \& Saitoh, T. R. 2011, ApJ, 735, 1

Wada, K. \& Koda, J. 2001, PASJ, 53, 1163

Walker, I. R., Mihos, J. C. \& Hernquist, L. 1996, ApJ, 460, 121

Wegg, C. \& Gerhard, O. 2013, MNRAS, to appear (arXiv1308.0593)

Weiland, J. L., et al. 1994, ApJL, 425, L81

Weinberg, M. D. 1985, MNRAS, 213, 451

Weinberg, M. D. 1991, ApJ, 373, 391

Weinberg, M. D. 1998, MNRAS, 297, 101

Weinberg, M. D. \& Katz, N. 2002, ApJ, 580, 627

Weinberg, M. D. \& Katz, N. 2007, MNRAS, 375, 425

Weiner, B. J., Williams, T. B., van Gorkom, J. H. \& Sellwood, J. A. 2001, ApJ, 546, 916

Wielen, R. 1977, A\&A, 60, 263

Wilson, M. L., et al. 2011, MNRAS, 413, 2235

Wyse, R. F. G. 2009, In "The Galaxy Disk in Cosmological Context", IAU Symposium 254. Eds. J. Andersen, J. Bland-Hawthorn \& B. Nordström (Cambridge, Cambridge University Press) p. 179 (arXiv:0809.4516)

Yoachim, P. \& Dalcanton, J. J. 2006, AJ, 131, 226

Yoachim, P., Roškar, R. \& Debattista, V. P. 2012, ApJ, 752, 97

Yong, D., Carney, B. W. \& Friel, E. D. 2012, AJ, 144, 95

York, D. G., 2000, AJ, 120, 1579

Yu, J-C., Sellwood, J. A., Pryor, C. Hou, J-L. \& Li, C. 2012, ApJ, 754, 124

Zang, T. A. 1976, PhD. thesis, MIT

Zánmar Sánchez, R., Sellwood, J. A., Weiner B. J. \& Williams, T. B. 2008, ApJ, 674, 797

Zibetti, S., Charlot, S. \& Rix, H.-W. 2009, MNRAS, 400, 1181 